\title{
Click and bioorthogonal chemistry: the future of active targeting of nanoparticles for nanomedicines?
}

Ludivine Taiariol, Carole Chaix ${ }^{*}$, Carole Farre and Emmanuel Moreau* 


\section{ABSTRACT}

Over the years, click and bioorthogonal reactions have been the subject of considerable research efforts. These high-performance chemical reactions have been developed to meet requirements not often provided by the chemical reactions commonly used today in the biological environment, such as selectivity, rapid reaction rate and biocompatibility. Click and bioorthogonal reactions have been attracting increasing attention in the biomedical field for the engineering of nanomedicines. In this review, we study a compilation of articles from 2014 to the present, using the terms "click chemistry and nanoparticles (NPs)" to highlight the application of this type of chemistry for applications involving NPs intended for biomedical applications. This study identifies the main strategies offered by click and bioorthogonal chemistry, with respect to passive and active targeting, for NP functionalization with specific and multiple properties for imaging and cancer therapy. In the final part, a novel and promising approach for "two step" targeting of NPs, called pretargeting (PT), is also discussed; the principle of this strategy as well as all the studies listed from 2014 to the present are presented in more detail.

\section{CONTENTS}

INTRODUCTION

1. Meta-analysis from the PubMed database using the term "click chemistry and nanoparticles".

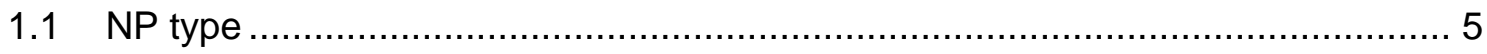

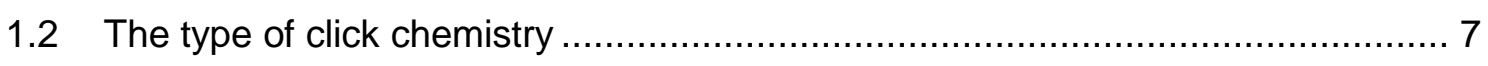

1.1.1. Copper(I)-catalyzed Azide-Alkyne [3+2] Cycloaddition (CuAAC) ............... 8

1.1.2. Strain-Promoted Alkyne-Azide Cycloaddition (SPAAC) .......................... 9

1.1.3. Photoinitiated thiol-ene reaction .................................................. 10

1.1.4. Inverse-electron-demand Diels-Alder [4+2] (IEDDA) ...........................10

1.3 The role of click chemistry in NP functionalization ....................................... 10

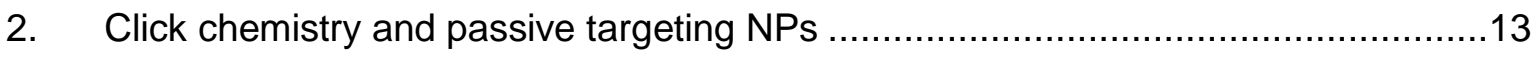

2.1 Passive targeting with payload encapsulation or complexation NPs ..................13

2.1.1 Encapsulation approaches ........................................................... 15

2.1.2 Complexation approaches............................................................17

2.2 Passive targeting with surface-functionalized NPs .....................................17

2.2.1 Surface functionalization with anticancer drugs ..................................17

2.2.2 Surface functionalization with imaging agents ......................................21

3. Influence of active targeting using click chemistry on tumor accumulation and

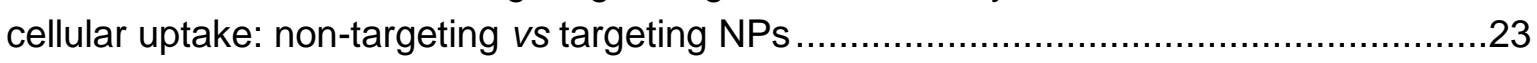

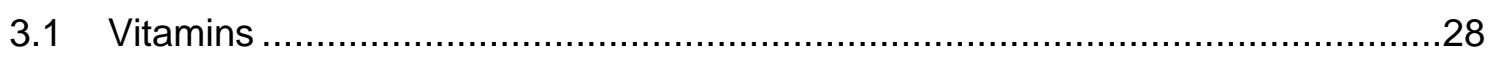

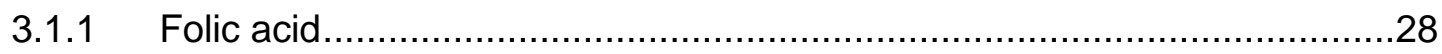

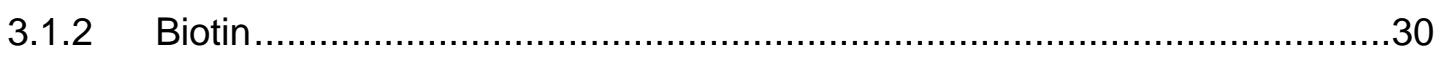

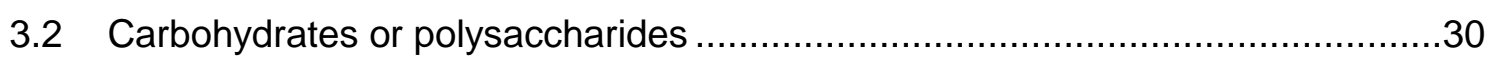

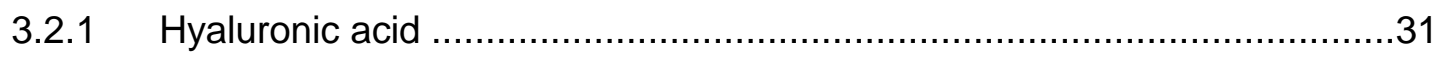

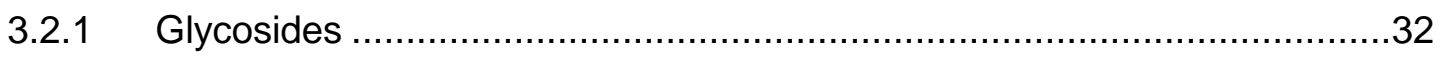




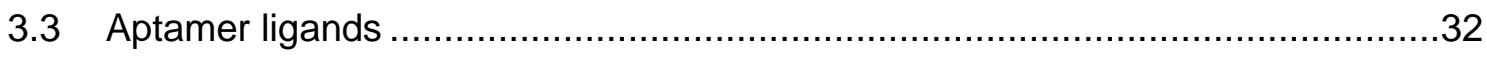

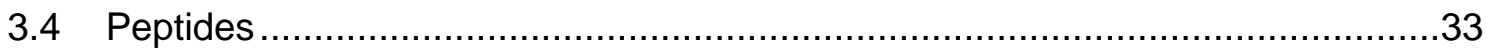

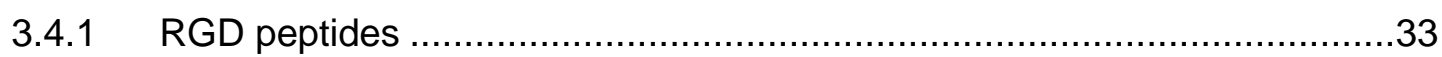

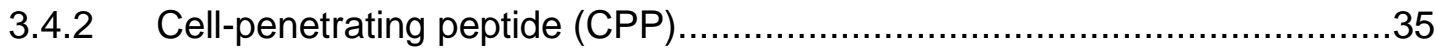

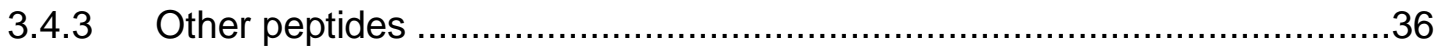

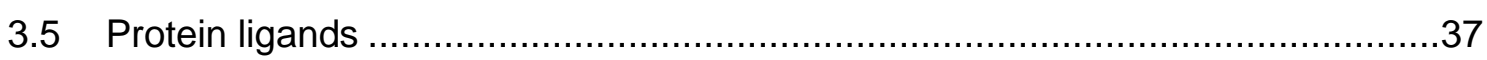

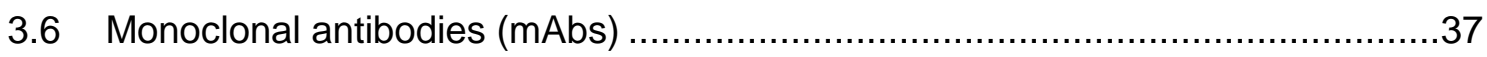

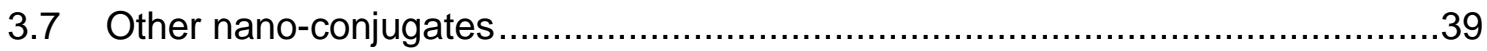

4. Bioorthogonal chemistry and pretargeting (PT) systems for NP delivery ................40

4.1 PT approaches with ligands as targeting agents .......................................41

4.2 PT approaches with NPs as a "platform" ..................................................47

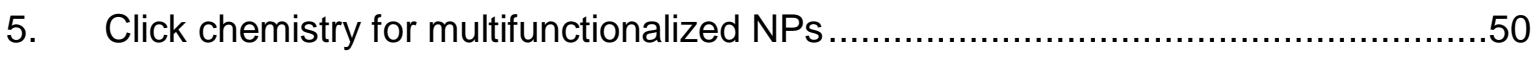

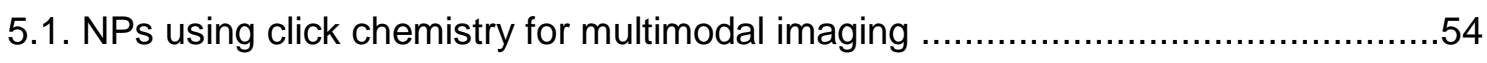

5.2. NPs using click chemistry for a theranostic approach .....................................56

5.3. NPs using click chemistry for imaging modality and a theranostic approach.........60

6. Nanoparticles, click chemistry and protein corona ...............................................61

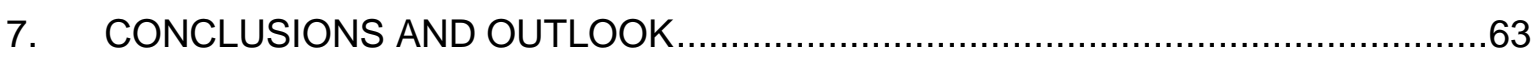

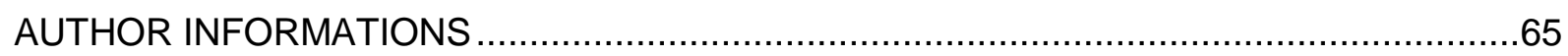

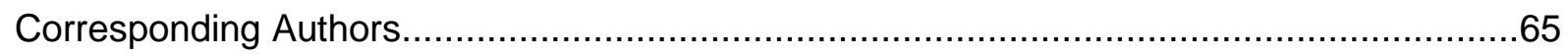

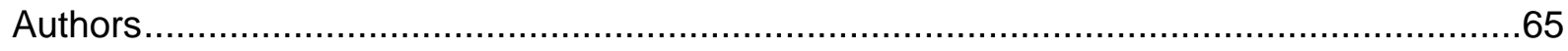

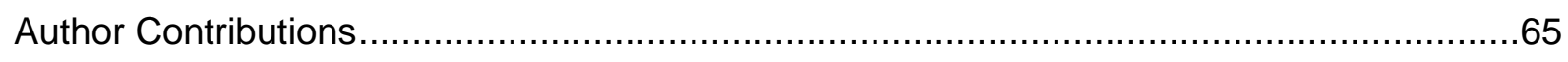

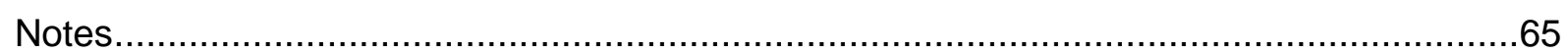

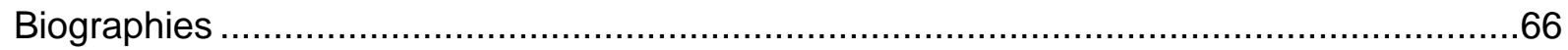

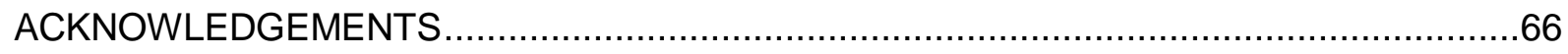

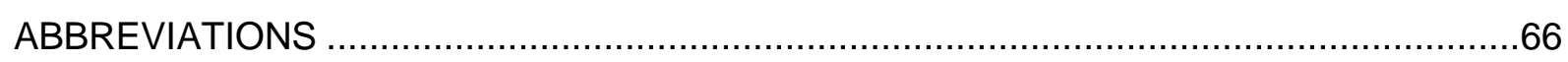

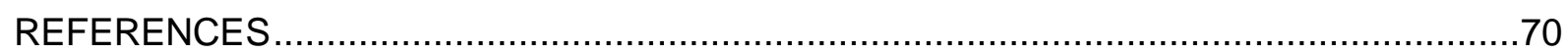

\section{INTRODUCTION}

Nanotechnologies are an integral part of our daily life, whether at work, at home or on holiday. An emerging development strategy has made a place for nano-based ingredients (nanomaterials) in consumer markets, such as paints, building materials, cosmetics, food, automobiles, electronics, pharmaceuticals, energy and materials, to name but a few. ${ }^{1}$ The ISO/TS 800004-1 (2015) standard defines a nanomaterial as a "material with any external dimension in the nanoscale (size range from approximately 1-100 nm) or having an internal structure or a surface structure in the nanoscale" and providing unique physicochemical properties (e.g. large surface area, high loading capacity, controlled size and shape). We can distinguish two main categories of nanomaterials in this nanoscale: (i) nano-objects (nanoparticles (NPs), nanofibers, nanotubes and nano-sheets); and (ii) nano-structured materials (aggregates and agglomerates of nano-objects, nanocomposites and nanoporous materials). ${ }^{2}$ Over the past two decades, NPs have emerged as promising tools in many 
scientific fields, including medicine. Nanomedicine has revolutionized the treatment of a number of pathologies including atherosclerosis, ${ }^{3}$ cardiovascular $^{4}$ and neurological disorders, ${ }^{5}$ infectious diseases, ${ }^{6}$ diabetes ${ }^{7}$ and endocrine disorders, ${ }^{8}$ arthritis, ${ }^{9}$ osteoarticular pathologies ${ }^{10}$ and cancer. ${ }^{11,12}$ Moreover, $70 \%$ of nanomedical products in the aforementioned medical specialties concern oncology, with $31 \%$ being in phase III clinical trials ${ }^{2}$ in the fields of diagnosis, imaging and treatment. ${ }^{13,14}$ However, only 15 anticancer drugs have been released on the market since $2017 . .^{15}$ There are a number of reasons for this somewhat surprising situation. The first is that the results obtained in the preclinical stage can be different from those of the clinical stages because the EPR effect largely depends on the tumor microenvironment, which differs between models and individuals. Indeed, several works from the past decade indicate that only $0.7 \%$ of injected NPs are found in the tumors. ${ }^{15,16}$ The second reason is a lack of reproducibility, which can be attributed to multiple factors, including design study, protocol, material quality and purity.

In parallel to the large-scale development of nanomaterials, another revolution in the field of biology and medicine has been the advent of click and bioorthogonal chemistries. These highly effective chemical reactions have been developed to fulfil the stringent rate, selectivity and biocompatibility requirements for targeting and labelling biomolecules in biological media.

Click chemistry was first described in 2001 by Sharpless et al. as a highly selective reaction occurring in mild aqueous conditions, providing good yields and favorable reaction rates compared with traditional reactions. ${ }^{17,18}$ For the development of organic molecules, it represents a means of obtaining a set of powerful, selective, and modular building blocks, such as an azide and an alkyne, that work on both small and large scales. Two important features make the click reaction a new approach in pre-clinical and clinical studies. Firstly, the functional groups of the reactant and those of the product do not interact with the functional biomolecules. Secondly, this kind of reaction takes place easily under mild conditions and in aqueous solvents, providing the best yields and highest rates. Shweta Verma recently defined click chemistry as "an interesting and novel approach to drug discovery, materials science, bioconjugation, radiochemistry and nanoscience. It meets an ever-increasing need for rapid reaction, as it fulfils the criterion of ideal synthesis: efficiency, versatility, selectivity and high yield with a variety of starting materials". ${ }^{19}$ Historically, the first so-called bioorthogonal reaction developed in vivo was the Staudinger reaction between an azide group and a phosphine that occurred under aqueous conditions with no toxic catalyst. ${ }^{20}$ However, its reaction kinetics were too slow, which led to the development of strainpromoted azide-alkyne cycloaddition (SPAAC) and inverse electron-demand Diels-Alder (iEDDA) reactions, thus allowing for rapid and specific covalent bond formation under aqueous conditions without the need for toxic catalysts. In the field of biomedical research, in particular, this has opened a new paradigm, proving that artificial chemical reactions can take place on cell surfaces, in the cytosol of cells or inside the body, which is not easily achieved with toxic catalysts. ${ }^{21}$ Based on these observations and new advances in chemical synthesis, we wanted to investigate whether click chemistry has also had a major impact in the field of the nanoparticle (NP), and particularly nanomedicine for oncology.

To determine the role of click chemistry in the world of NPs, we first used the search term "bioorthogonal chemistry nanoparticles" in the PubMed database, because bioorthogonal 
reactions are normally defined as copper-free and therefore facilitate nanomedicine applications. Surprisingly, we identified only 167 articles for the period 2010-2020 and only 139 from 2014 to recently. Hence, we used the search term "click chemistry nanoparticles" and identified 1,170 articles, including 848 from 2014. We suggest that this major difference between search terms might be attributed to inappropriate use of the term "click" by authors whose articles concern bioorthogonal chemistry. Indeed, several articles were common to both searches while the other 37 , included in this analysis, did not appear in the "click chemistry" search.

Finally, the most representative panel of 848 articles was classified according to several criteria such as NP type (liposomes, dendrimers, quantum dots, silica NPs, gold NPs, etc.), the nature and role of click chemistry ("decoration" or molecule grafting, passive targeting, and in case of active targeting, the nature of the targeting molecules used). This resulted in a selection of 319 articles; the others were excluded because they did not fall within the field of oncology or because of a lack of information. In this review, we describe new modification possibilities for NPs and highlight the contribution of click chemistry (i.e. copper catalyst and copper catalyst-free reactions) to the registration of functional organic and inorganic NPs for cancer imaging and therapy. This includes recent applications in which the reactions themselves have been used for functionalization and for coupling ligands to NPs. Finally, we discuss the impact and future of click and bioorthogonal chemistries in the area of NPs.

\section{Meta-analysis from the PubMed database using the term "click chemistry and nanoparticles"}

In order to gain a better insight into the possible impact of click chemistry in nanomedicine, we conducted a detailed analysis of these 319 articles. Firstly, we analyzed whether all types of nanoparticles were concerned by click chemistry and which class of click chemistry was the most used (CuAAC, SPAAC or IEDDA). We also analyzed the different articles to determine the different applications of click chemistry in the world of nanomedicine.

\subsection{NP type}

Trends may be observed in data concerning the different types of nanomaterials used for click chemistry (Figure). Polymeric NPs and dendrimers tend to be the most commonly used, representing approximately $72 \%$ of the references when combined as organic nanomaterials, unlike micelles and liposomes, which represent only $16 \%$. This is probably due to the wide use of polymers to coat NPs in order to make them biocompatible and easily functionalizable on the surface. Polymers are soft materials that circulate more easily in blood vessels and prevent aggregation and clogging risks. Furthermore, dendrimers are excellent agents for in vivo diagnostics involving imaging and therapy because they have a large number of reactive groups to be functionalized and high solubility in the aqueous phase. This results in a high functionalization capacity for targeted cancer treatments and bioimaging devices. ${ }^{22-25}$ The high solubility of liposomes and their rapid elimination from systemic circulation could be an advantage for biological applications. 

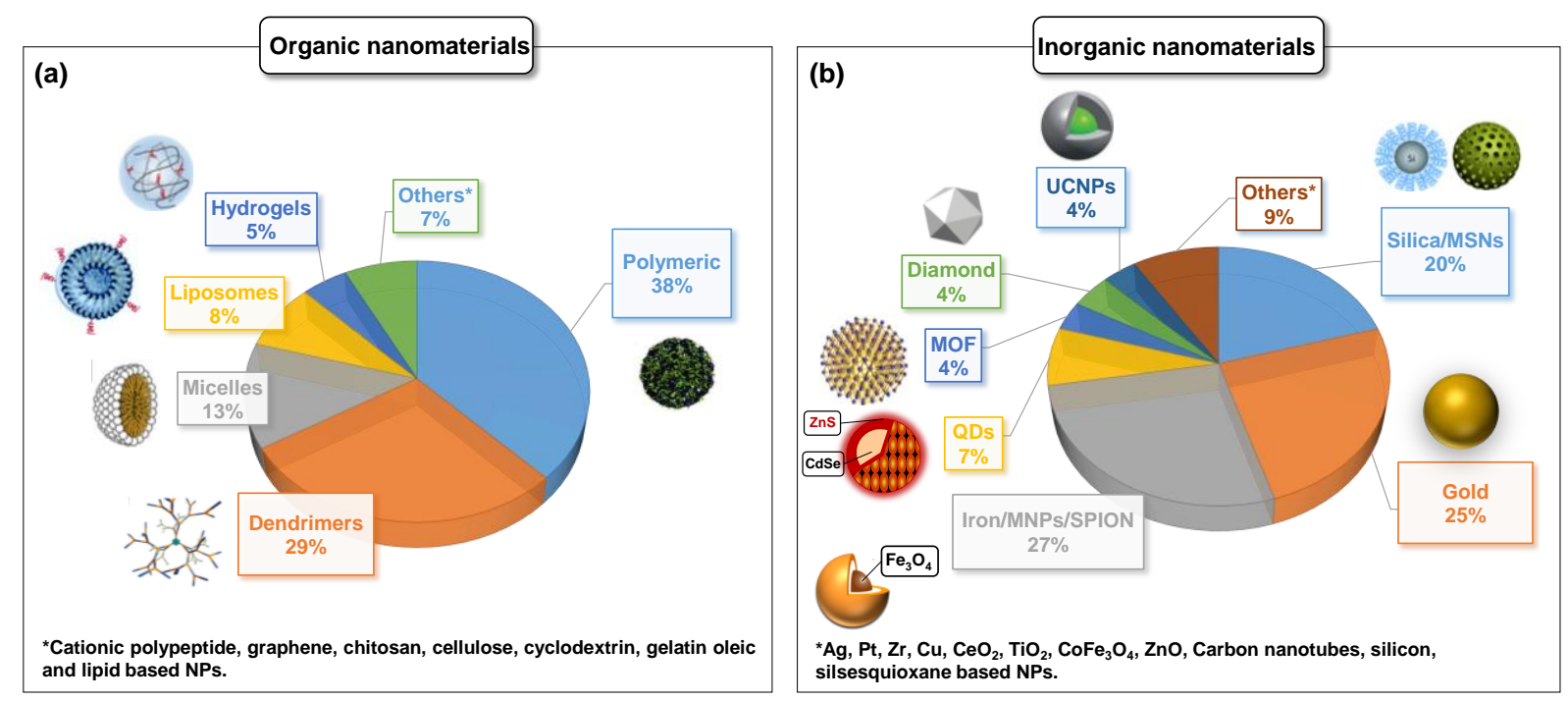

Figure 1. Analysis of the different types of NPs using the search term "click chemistry and nanoparticles" in articles published from 2014. (a) Organic materials, (b) Inorganic materials.

Silica $\left(\mathrm{SiO}_{2}\right)$, gold $(\mathrm{Au})$ and iron oxide $\left(\mathrm{Fe}_{3} \mathrm{O}_{4}\right)$ NPs account for $75 \%$ of inorganic NPs and offer an interesting click chemistry modification platform for both imaging and therapy. AuNPs are widely used as multifunctional NPs because they can be decorated by targeting, therapeutic and fluorescent molecules and, at the same time, they can be used for photothermal (PTT) or photodynamic therapy (PDT) thanks to their surface plasmon resonance properties ${ }^{26}$ and as contrast agents for $\mathrm{CT}$ imaging. ${ }^{27}$ Silica is a material of choice for biomedical applications because of its stability, versatility and biocompatibility. This material can also be applied as a coating to a number of metallic systems, such as $\mathrm{Fe}_{3} \mathrm{O}_{4}$ NPs and AuNPs, to improve their biocompatibility. ${ }^{28-31}$

The stable $\mathrm{SiO}_{2}$ NP category includes mesoporous silica NPs (MSNs), which are commonly employed for their capacity to encapsulate or absorb cargo (e.g. anticancer drugs, siRNA, plasmid DNA and peptides) into large porous volumes $\left(>0.9 \mathrm{~cm}^{3} / \mathrm{g}\right)$ for stimuli-responsive drug release. ${ }^{32}$ MSNs are notably used to improve drug accumulation and the therapeutic effects of insoluble hydrophobic anticancer drugs. ${ }^{33}$ They provide adaptable pore size, a wide surface area for functionalization ( $900 \mathrm{~m}^{2} / \mathrm{g}$ ), and have high thermal and chemical stability. ${ }^{34,35}$

These diverse properties highlight the advantages of this gatekeeper for imaging, ${ }^{36}$ targeting ${ }^{37}$ and therapy. ${ }^{38} \mathrm{Fe}_{3} \mathrm{O}_{4} \mathrm{NPs}$, also referred to as "Ultra Small SuperParamagnetic Iron Oxide" NPs (USPION, diameter $<50 \mathrm{~nm}$ ) or "SuperParamagnetic Iron Oxide" NPs (SPION, diameter with a range of $50-500 \mathrm{~nm}$ ), have attracted a great deal of interest in the field of click chemistry due to their numerous biomedical applications. These nanomaterials have magnetic properties that have been largely exploited for magnetic-resonance imaging $(\mathrm{MRI}){ }^{39}$ sometimes combined with positron-emission tomography (PET) ${ }^{40}$ and/or optical imaging. ${ }^{41}$ In therapeutic approaches, they are used either for the delivery of conventional gene $^{42}$ or chemotherapies, ${ }^{43}$ or for the hyperthermal destruction of cancer cells. ${ }^{44}$ Their surface, usually coated with a matrix of silica, polysaccharides (e.g., dextran) or polymers (e.g., polyethylene glycol, PEG), is suitable for functionalization with a wide variety of targeting or therapeutic molecules. ${ }^{45,46}$ 


\subsection{The type of click chemistry}

The surface chemical modification of a wide variety of organic and inorganic nanomaterials has been extensively investigated over the past few years. The incorporation of chemical functions onto the surface of the NPs allows subsequent functionalization to gain either stability or specific properties. Oligonucleotides, monoclonal antibodies (mAbs), proteins and peptides, for instance, can be attached by electrostatic interactions or by covalent linkage. Thiol, phosphine oxide, phosphonate and carboxylate groups are employed too, particularly in metal oxide NPs. ${ }^{47}$ Surface modification can usually be carried out by either multi-step or one-step functionalization. ${ }^{48}$ Chemical reactions, such as carbodiimide-mediated coupling between carboxyl and amine, succinimidyl ester-amine and maleimide-thiol are commonly used standard bioconjugation reactions (Figure). ${ }^{49,50}$ More detailed information on functionalization strategies for the incorporation of multiple functions and/or biomolecules on the surface of nanomaterials is presented in the review by De Crozals et al..$^{34}$

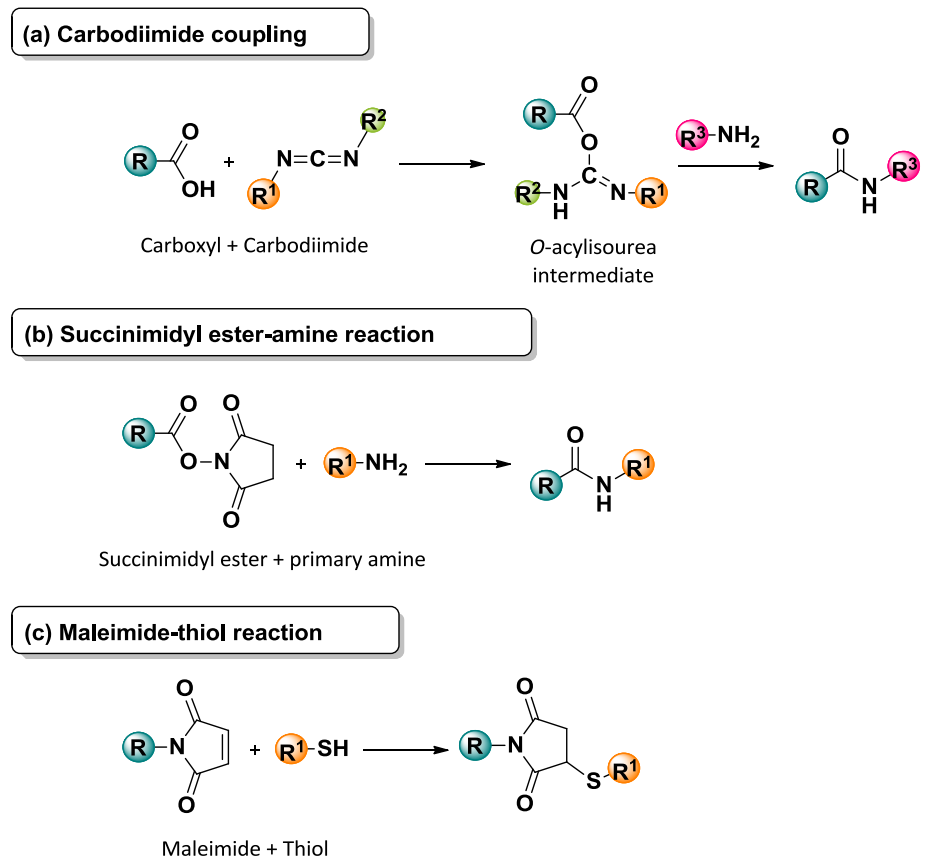

Figure 2. Traditional reactions for NP bioconjugation. (a) Carbodiimide coupling, (b) Succinimidyl ester-amine reaction, (c) Maleimide-thiol reaction. In the schemes, the symbols $\mathbf{R}, \mathbf{R}^{1}, \mathbf{R}^{2}$ and $\mathbf{R}^{3}$ refer to any substituent.

Conventional reactions, such as amine-activated ester acylations, are widely used for derivatizing NPs with proteins in vitro. Nevertheless, reaction conditions such as temperature and $\mathrm{pH}$ must be considered. For instance, the use of carbodiimide coupling reactions can be subject to hydrolysis after formation of the reactive intermediate o-acylisourea using 1-ethyl3-(3-dimethylaminopropyl)carbodiimide (EDC), thus limiting overall yields. ${ }^{51}$ Furthermore, since the latter is only slightly soluble, the number of activated carboxyl groups present on the NPs must be controlled to avoid the risk of losing colloidal stability and encouraging aggregation. The agent $\mathrm{N}$-hydroxysuccinimide (NHS) or the more hydrophilic agent $\mathrm{N}$ hydroxysulfosuccinimide (Sulfo-NHS) can be used in conjunction with EDC to improve the amino-group coupling yield to the activated ester. Coupling chemistries are not specific enough to ensure the binding strength of conjugations and biomolecule orientation, which are 
essential for biological functions. For instance, mAbs and proteins possess many different amino acid functional groups (e.g., aspartic acid, glutamic acid) which can react with each other, producing undesirable byproducts and competing with NP-protein binding. The development of more specific reactions could help to control specific binding sites. Similarly, multifunctional NPs with several functional molecules often generate non-specific adsorption and uncontrolled grafting, possibly causing unexpected hydrophobic or electrostatic interactions. ${ }^{34}$ Reactions may occur between the functional group and the particle surface, for instance back-bonding of amino or ammonium groups with negatively charged NPs. ${ }^{48}$ It is therefore necessary to develop simple but more efficient reactions to produce heteroatomlinked molecular systems in a specific manner. In this context, click chemistry has demonstrated its superior ability to provide all these properties, compared with traditional chemical methods. This attractive chemistry is the focus of this review, and the different types of click chemistry commonly used are presented in more detail in the following sections.

\subsubsection{Copper(I)-catalyzed Azide-Alkyne [3+2] Cycloaddition (CuAAC)}

Our meta-analysis reveals that the most documented click reaction for NPs in the literature is copper(I)-catalyzed azide-alkyne cycloaddition (CuAAC), representing more than the half of the listed publications (Figurea).

(a)

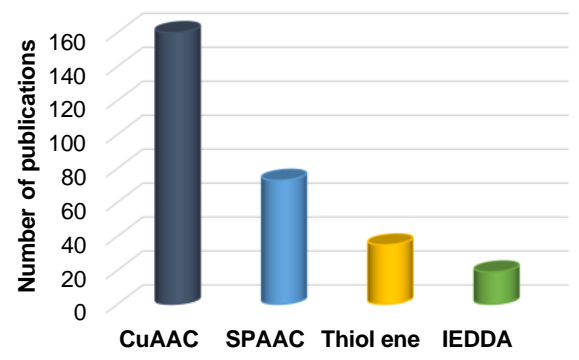

(b)

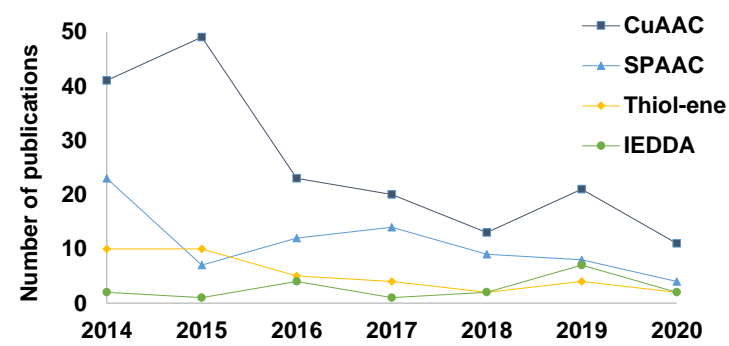

(c)

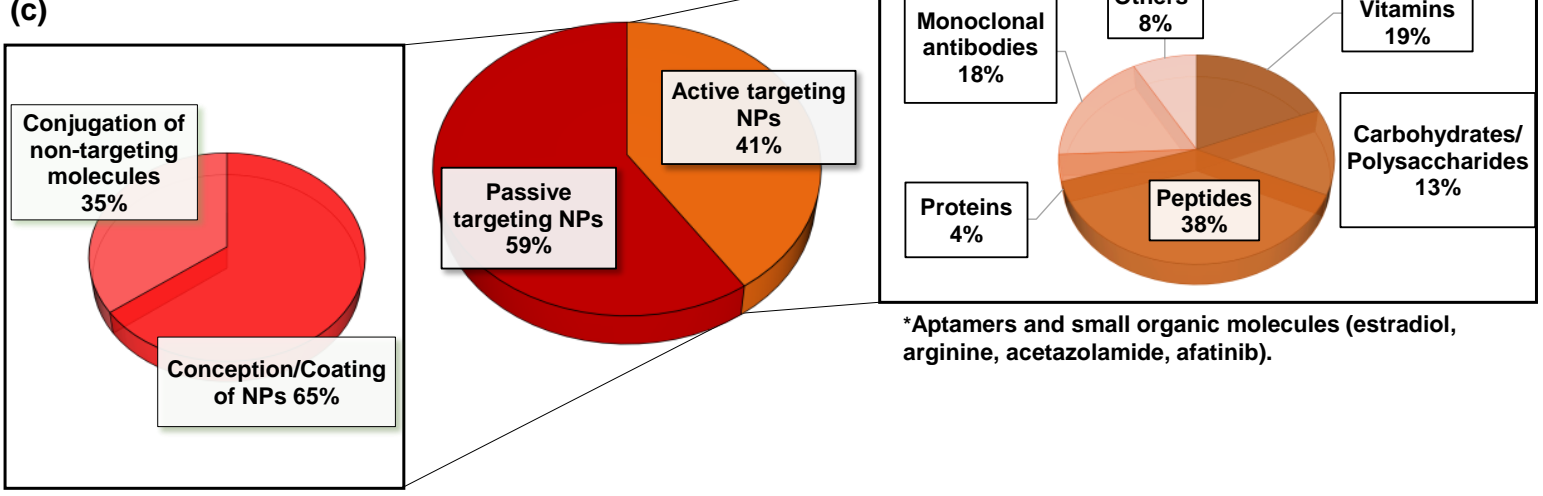

Figure 3. Graphic representations of CuAAC, SPAAC, thiol-ene and IEDDA applications for NPs according to publications from 2014 to 2020. (a) Total number of publications for each type of click chemistry, (b) Evolution over time of the total number of publications for each type of click chemistry, (c) Proportion of publications concerning active and passive targeting NPs.

In recent years, a number of studies have investigated Cu-catalyzed cycloaddition and demonstrated its effectiveness for the surface modification of organic and inorganic NPs with molecules and biomolecules. CuAAC between an azide and a terminal alkyne generates a 
stable 1,2,3 triazole ${ }^{17}$ with a fast reaction speed (second-order rate constant $k_{2}$ up to $10^{4} \mathrm{M}^{-}$ $\left.{ }^{1} \mathrm{~s}^{-1}\right)^{52}$ and reproducible results in physiological conditions (Figure). ${ }^{18}$ Modification of the NP surface for specific cell interactions generally involves a first step allowing chemical binding of an alkyne or azide on the surface, followed by a second step to add the biomolecules. However, the use of CuAAC has been in decline since 2016 (Figureb). This could be due to the metal catalyst of the reaction, $\mathrm{Cu}(\mathrm{I})$, which strongly limits its use in biological settings because of the cytotoxicity induced by possible interaction or chelation with biomolecules ${ }^{53}$ or in the preparation of imaging tools because of the fluorescence reduction for certain proteins (e.g. green fluorescent protein, GFP) and quantum dots (QDs). ${ }^{52,54,55} \mathrm{CuAAC}$ can also form in situ complexes with some compounds, possibly making it more difficult to quantify the reaction product and purity. ${ }^{56}$ For example, Williams and co-workers describe a reduction of cell viability due to inadequate methods for removing all the $\mathrm{Cu}$ ions after functionalizing nanoporous silica NPs with polysialic acid. ${ }^{57}$ Moreover, $\mathrm{Cu}(\mathrm{I})$ may be unstable in aqueous solutions at this degree of oxidation. This reaction is not suitable for in vivo coupling.

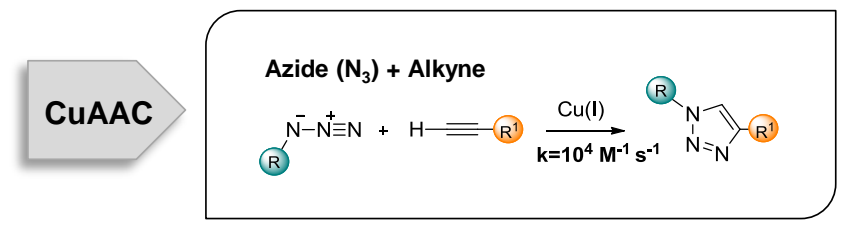

Figure 4. Copper(I)-catalyzed azide-alkyne [3+2] cycloaddition (CuAAC) reaction scheme. In the scheme, the symbols $\mathbf{R}$ and $\mathbf{R}^{\mathbf{1}}$ refer to any substituent.

\subsubsection{Strain-Promoted Alkyne-Azide Cycloaddition (SPAAC)}

The drawbacks of copper have led many research projects to turn to biocompatible catalystfree click reactions, such as SPAAC, developed in 2010 by Bertozzi and coworkers. ${ }^{58}$ Between 2014 and 2020, approximately 20\% of the studies recorded concern SPAAC cycloaddition (Figurea), which consists in using strain-stable carbocyclic alkynes, called cyclooctynes, to allow the reaction with azide moieties (Figure). SPAAC does not require a catalyst and is inert towards biomolecule functional groups and other functions found in biological environments. However, the relatively slow reaction rate of azides $\left(\mathrm{N}_{3}\right)$ with dibenzocyclooctyne groups (DBCO) $\left(k_{2}=0.2-0.5 \mathrm{M}^{-1} \mathrm{~s}^{-1}\right)^{52}$ can limit in vivo applications. Attention therefore turned to another stable aliphatic cyclooctyne called bicyclo[6.1.0]nonyne (BCN), which offers accelerated reaction rate constants up to $2.9 \mathrm{M}^{-1} \mathrm{~s}^{-1}$. 59

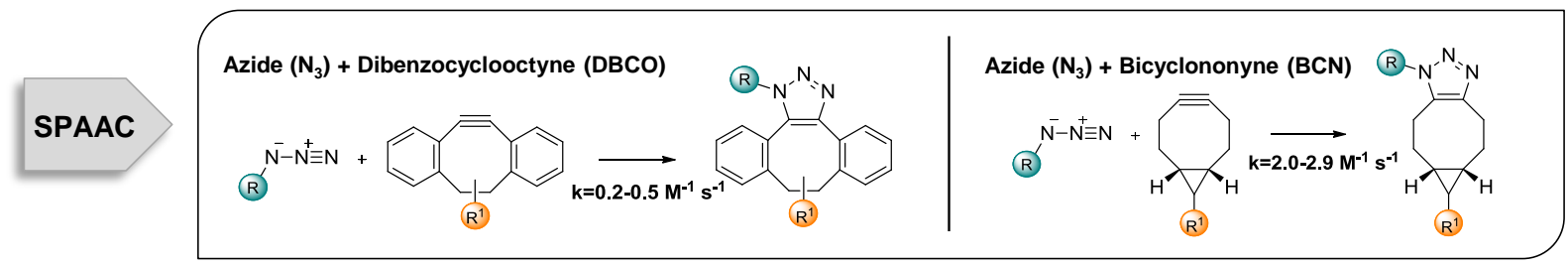

Figure 5. Strain-promoted alkyne-azide cycloaddition (SPAAC) reaction scheme. In the scheme, the symbols $\mathbf{R}$ and $\mathbf{R}^{1}$ refer to any substituent. 


\subsubsection{Photoinitiated thiol-ene reaction}

The thiol-ene reaction has also been investigated by scientists for NP functionalization..$^{60,61}$ Thiol-ene involves a reaction between a thiol $(\mathrm{SH})$ and an alkene group to form an alkyl sulfide and can be initiated by free-radical addition (photoinitiation, thermal or redox) (Figure). This reaction is mainly involved in the synthesis of dendrimers and polymers ${ }^{62}$ due to the fact that it can effectively perform radical-based photopolymerization reactions for staged growth (propagation and chain transfer) and chain growth (polymerization), combining all the benefits of click chemistry. ${ }^{63}$

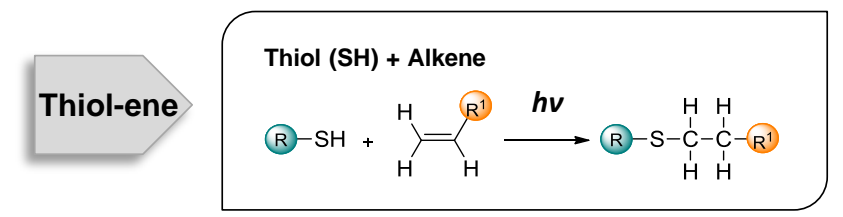

Figure 6. Photoinitiated thiol-ene reaction scheme. In the scheme, the symbols $\mathbf{R}$ and $\mathbf{R}^{\mathbf{1}}$ refer to any substituent.

\subsubsection{Inverse-electron-demand Diels-Alder [4+2] (IEDDA)}

IEDDA cycloaddition occurs between an electron-rich dienophile, such as trans-cyclooctene (TCO) or norbornene, and an electron-poor diene called 1,2,4,5-tetrazine (Tz). This is another click reaction of interest for nanomaterials with an excellent reaction rate $\left(k_{2}=10^{4} \mathrm{M}^{-}\right.$ $\left.{ }^{1} \mathrm{~s}^{-1}\right)$. Although IEDDA is the fastest click chemistry reaction, it is rarely described for NP functionalization (Figurea,b and 7), remaining most commonly used for two-step protocols (i.e. pretargeting strategies) to deliver NPs to the cells (see Part $\mathbf{4}$ of this manuscript). ${ }^{64,65}$

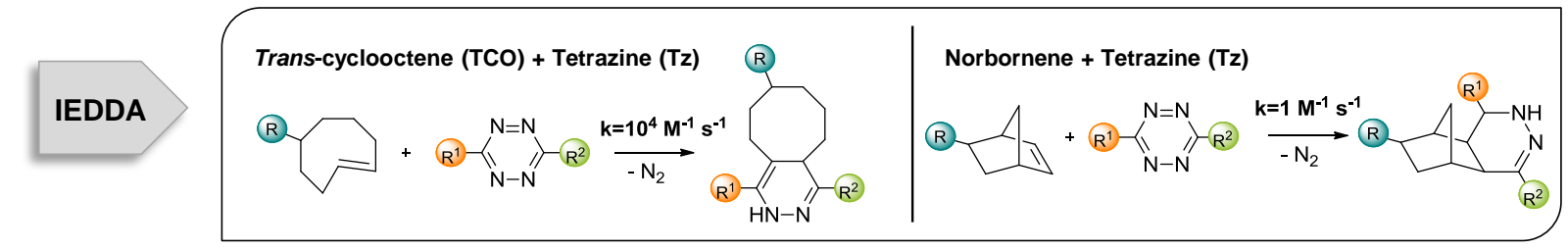

Figure 7. Inverse-electron-demand Diels-Alder [4+2] (IEDDA). In the scheme, the symbols $\mathbf{R}, \mathbf{R}^{1}$ and $\mathbf{R}^{\mathbf{2}}$ refer to any substituent.

\subsection{The role of click chemistry in NP functionalization}

Several trends can be observed in the six-year analysis concerning the field of nanomedicines (Figurec). First of all, it should be noted that $41 \%$ of the click chemistry reactions described above are used for active targeting, allowing NPs to achieve specific cell targeting prior to functionalization by several ligands/biomarkers of interest such as peptides, vitamins, mAbs, carbohydrates and proteins. ${ }^{66,67} 59 \%$ of publications describe click reactions for passive targeting. $65 \%$ of the articles describe proofs of concept (no particular applications) for NP design (e.g. dendron assembly for dendrimers, polymer assembly for polymeric NPs) and coating (for example with polymers or polyethylene glycol units). The 
conjugation of non-targeting molecules, firstly for encapsulation (e.g. polymers, polyethylene glycols, stimuli-responsive molecules) or complexation (e.g. amylose and carboxylic functions), and secondly for surface functionalization (e.g. anti-cancer drugs, contrast agents and stimuli-responsive linkers) constitutes the remaining $35 \%$ of the listed articles (Figure). 


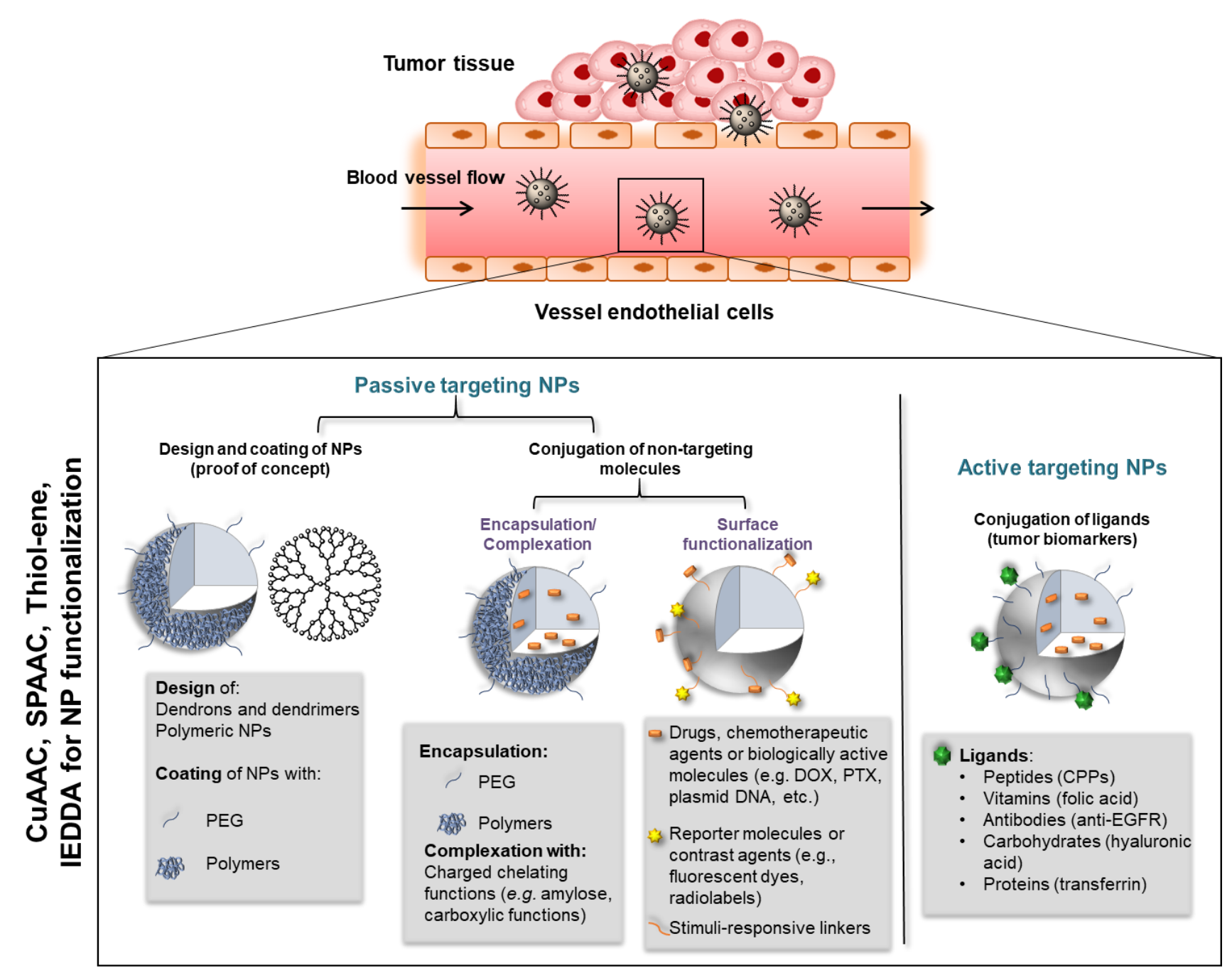

Figure 8. Different applications of click chemistry reactions for NP functionalization. 


\section{Click chemistry and passive targeting NPs}

Two main strategies are reported to achieve site-specific delivery of NPs: active targeting and passive targeting, which is also called the EPR effect, described for the first time in 1986 by Maeda and co-workers. ${ }^{68}$ Most nanomedicine research has been devoted to passive targeting. Indeed, the tumor microenvironment (TME) has numerous functional abnormalities which enable NPs in the 20-200 nm size range to diffuse into tissues and gather in the tumor region. Tumor tissue includes an unnatural vasculature barrier that is poorly organized and irregular in shape; once the NPs have passed through the intercellular gaps in this barrier (i.e. extravasation), weak lymphatic drainage allows them to remain in the tumor. ${ }^{16,69,70}$ For most of the articles investigated, click chemistry was used to develop passive targeting NPs to incorporate therapeutic agents either (i) by encapsulation/complexation and/or (ii) by adding them to the surface of the nanoparticles. These two aspects are more comprehensively developed in the following sections.

\subsection{Passive targeting with payload encapsulation or complexation NPs}

Based on the EPR effect for effective transfer into tumors, NPs were first exploited because of their capacity to encapsulate drugs. The first passively targeted nanomedicine approved by the FDA in 1995 for clinical use, now considered to be one of the most competitive chemotherapeutic systems, was PEGylated liposome embedding doxorubicin (DOXIL ${ }^{\mathrm{TM}}$ in the US, Caelyx ${ }^{\mathrm{TM}}$ in other countries). This nanocarrier has been found to increase blood circulation time and has the potential to enhance tumor accumulation in a broad range of cancers (sarcoma, breast, myeloma and ovarian). ${ }^{71}$ Other successful liposomal drug delivery methods are also now used in clinics, such as Abraxane ${ }^{\mathrm{TM}}$, an albumin-bound-particle, for metastatic breast cancer and pancreatic adenocarcinoma. ${ }^{72}$ Liposomal encapsulation of paclitaxel produces more effective response rates than the free drug. Similarly, DaunoXome $^{\mathrm{TM}}$ and Myocet ${ }^{\mathrm{TM}}$, non-PEGylated $50 \mathrm{~nm}$ liposomal daunorubicin and $150 \mathrm{~nm}$ liposomal doxorubicin, respectively, have also been developed as NPs to enhance the EPR effect. Among cancer therapies, paclitaxel-loaded polymeric micelles (i.e. Genexol®-PM) have been approved in Korea for breast, lung and pancreatic cancers. ${ }^{73}$ As mentioned above, paclitaxel (PTX), daunorubicin, doxorubicin (DOX) are the most commonly embedded anti-cancer drugs; however, others have recently emerged, such as cisplatin (CDDP), methotrexate (MTX) and docetaxel. Over the past two decades, various nanocarrier systems encapsulating chemotherapeutic drugs have been developed to improve anti-cancer effects, while reducing their potential toxicity. There are major advantages to encapsulation systems: i) they extend the half-life of the loaded drug, ii) they increase drug exposure to tumors by exploiting the EPR effect, iii) they improve bioavailability and therefore the therapeutic index. The increasing number of preclinical in vitro and in vivo studies describing passively and non-targeted nanocarriers is encouraging in terms of transfer to the clinical stage.

In this study, the articles listed highlight the fact that click chemistry contributes to the encapsulation of therapeutic agents by entrapping with PEG polymers, or to the formation of dendrimers and polymeric NPs, and to drug complexation by incorporating molecules such as amylose or carboxylic functions (Table 1). Generally speaking, the encapsulated therapeutic drugs are released by endogenous or exogenous stimuli, such as $\mathrm{pH}$, 
temperature, redox, enzyme, or by a combination of triggers for efficient tumor accumulation. $^{74}$

MSNs, polymeric NPs and micelles are the most commonly employed NPs described in the publications as $\mathrm{pH}$-responsive drug carriers using click chemistry. They are usually designed to encapsulate therapeutic molecules and to be taken up by an endocytosis route, then hydrolyzed by endosomes or lysosomes, resulting in higher toxicity for tumor cells than free drugs.

Table 1. Overview of passively targeting NPs (as drug carriers) using click chemistry for the encapsulation and complexation of drugs, from 2014 to nowadays.

\begin{tabular}{|c|c|c|c|c|c|c|}
\hline $\begin{array}{l}\text { Type of click } \\
\text { chemistry and use } \\
\text { (grafted click } \\
\text { function) }\end{array}$ & $\begin{array}{l}\text { Type of NPs } \\
\text { (grafted click } \\
\text { function) }\end{array}$ & Drug loading & $\begin{array}{l}\text { Release } \\
\text { mechanism }\end{array}$ & Effects & $\begin{array}{l}\text { In vitro/In } \\
\text { vivo studies }\end{array}$ & Refs. \\
\hline $\begin{array}{l}\text { CuAAC, formation of } \\
\text { micelles }\end{array}$ & $\begin{array}{l}\text { Nanomicelles } \\
\left(\text { Alkyne/ } \mathbf{N}_{3}\right)\end{array}$ & $\begin{array}{l}\text { DOX, MPLA } \\
\text { adjuvant }\end{array}$ & n.d & $\begin{array}{l}\text { Cytotoxicity and } \\
\text { anticancer } \\
\text { activity }\end{array}$ & yes/yes & 75,76 \\
\hline $\begin{array}{l}\text { CuAAC, synthesis of } \\
\text { dendrimers }\end{array}$ & $\begin{array}{l}\beta \text {-cyclodextrin } \\
\text { dendrimers }\end{array}$ & MTX & Acidic release & Cytotoxicity & yes/no & 77 \\
\hline $\begin{array}{l}\text { CuAAC, ring-opening } \\
\text { metathesis } \\
\text { copolymerization } \\
\text { (ROMP) }\end{array}$ & Polymeric & Vorinostat & Acidic release & $\begin{array}{l}\text { Cytotoxicity and } \\
\text { anticancer } \\
\text { activity }\end{array}$ & yes/yes & 78 \\
\hline $\begin{array}{l}\text { CuAAC, incorporation of } \\
\text { labile Schiff base for } \\
\text { acidic control release }\end{array}$ & Polymeric & DOX & Acidic release & Cytotoxicity & yes/no & 79 \\
\hline $\begin{array}{l}\text { CuAAC, incorporation of } \\
\mathrm{Pt}(\mathrm{IV}) \text { prodrug (Alkyne) }\end{array}$ & Nanomicelles $\left(\mathbf{N}_{\mathbf{3}}\right)$ & $\mathrm{Pt}(\mathrm{IV}), \mathrm{DOX}$ & $\begin{array}{l}\text { Endosome } \\
\text { degradation }\end{array}$ & Cytotoxicity & yes/no & 80 \\
\hline $\begin{array}{l}\text { CuAAC, incorporation of } \\
\text { photo-responsive } \\
\text { molecules }\left(\mathbf{N}_{3} / \text { Alkyne }\right)\end{array}$ & MSNs ( $\mathbf{N}_{\mathbf{3}} /$ Alkyne) & DOX & $\begin{array}{l}\text { Photo- } \\
\text { responsive } \\
\text { release }\end{array}$ & Cytotoxicity & yes/no & 81 \\
\hline $\begin{array}{l}\text { CuAAC, modification of } \\
\text { side chain polymeric } \\
\text { NPs with PEG (Alkyne) }\end{array}$ & Polymeric $\left(\mathbf{N}_{3}\right)$ & СРT & $\begin{array}{l}\text { Ester linker } \\
\text { hydrolysis }\end{array}$ & $\begin{array}{l}\text { Cytotoxicity and } \\
\text { anticancer } \\
\text { activity }\end{array}$ & yes/yes & 82 \\
\hline $\begin{array}{l}\text { CuAAC, surface } \\
\text { incorporation of PEG } \\
\text { (Alkyne) }\end{array}$ & $\mathrm{NMOFs}\left(\mathbf{N}_{\mathbf{3}}\right)$ & $\begin{array}{l}\text { Dichloroacetic } \\
\text { acid }\end{array}$ & $\begin{array}{l}\mathrm{pH}- \\
\text { responsive } \\
\text { release }\end{array}$ & Cytotoxicity & yes/no & 83 \\
\hline $\begin{array}{l}\text { CuAAC, incorporation of } \\
\text { amylose }\left(\mathbf{N}_{3}\right)\end{array}$ & $\begin{array}{l}\text { Cationic amylose- } \\
\text { based dendrimers } \\
\text { (Alkyne) }\end{array}$ & Plasmid DNA & n.d & $\begin{array}{l}\text { Gene } \\
\text { transfection } \\
\text { efficacy }\end{array}$ & yes/no & 84 \\
\hline $\begin{array}{l}\text { CuAAC, conjugation of } \\
\text { dextran }\left(\mathbf{N}_{3}\right)\end{array}$ & $\begin{array}{l}\text { Dextran-based } \\
\text { PAMAM dendrimers } \\
\text { (Alkyne) }\end{array}$ & Plasmid DNA & n.d & $\begin{array}{l}\text { Gene } \\
\text { transfection } \\
\text { efficacy }\end{array}$ & yes/no & 85 \\
\hline $\begin{array}{l}\text { CuAAC, incorporation of } \\
\text { amylose }\left(\mathbf{N}_{3}\right)\end{array}$ & $\begin{array}{l}\text { Cationic amylose- } \\
\text { based dendrons } \\
\text { (Alkyne) }\end{array}$ & Thrombin & n.d & $\begin{array}{l}\text { Hemostatic } \\
\text { activity }\end{array}$ & yes/yes & 86 \\
\hline $\begin{array}{l}\text { Thiol-ene, incorporation } \\
\text { of silyl ether produg } \\
\text { (Allyl) }\end{array}$ & MSNs (SH) & СРТ & Acidic release & Cytotoxicity & yes/no & 87 \\
\hline $\begin{array}{l}\text { Thiol-ene, incorporation } \\
\text { of carboxyl groups for } \\
\text { CDDP chelation }\end{array}$ & Dendrimers (Allyl) & CDDP, PTX & n.d & $\begin{array}{l}\text { Cytotoxicity and } \\
\text { anticancer } \\
\text { activity }\end{array}$ & yes/yes & 88 \\
\hline $\begin{array}{l}\text { Thiol-ene, incorporation } \\
\text { of carboxyl groups } \\
\text { (maleic anhydride) for } \\
\text { drug chelation }\end{array}$ & MSNs (SH) & $\mathrm{DACH}-\mathrm{Pt}$ & n.d & Cytotoxicity & yes/no & 89 \\
\hline
\end{tabular}


n.d: not determined, $\mathrm{N}_{3}$ : azide, SH: thiol, DOX: doxorubicin, MTX: methotrexate, CPT: camptothecin, CDDP: cisplatin, Pt(IV): Platinum(IV), DACH-Pt: dichloro(1,2-diaminocyclohexane)platinum(II), MSNs: mesoporous silica nanoparticles, NMOFs: metal-organic framework nanoparticles.

"Effects" indicate the NP effects on cancer cells in vitro and/or in vivo.

"In vitrolin vivo studies" indicate whether in vitro or in vivo tests were performed (yes) or not (no) in the article.

\subsubsection{Encapsulation approaches}

\section{Examples of CuAAC}

In a recent study, Mei et al. described azide and alkyne micellar nanocarriers embedding paclitaxel PTX, demonstrating an improvement of tumor retention and anti-tumor effects for the treatment of lymph node metastasis of breast cancer. ${ }^{75}$ After the $25 \mathrm{~nm}$ micelles (S-PTX) were accumulated in the tumor, catalysts (copper sulfate and sodium ascorbic acid) were directly injected intratumorally to initiate CuAAC between the micelles. This process enabled the formation of larger micelles (S-PTX $(+), 100 \mathrm{~nm}$ ) which were better retained in the tumor by the EPR effect 12 hours after injection compared with S-PTX and pre-formed larger micelles (L-PTX) $(p<0.1)$. These S-PTX $(+)$ nanomicelles showed significant antitumor activity with $76 \%$ tumor suppression vs $49 \%$ for L-PTX and $57 \%$ for S-PTX.

The same authors also applied this strategy for immunochemotherapy with co-delivery of doxorubicin DOX and monophosphoryl lipid A (MPLA), and demonstrated effective tumor volume suppression in combination with anti-PD-L1 (M-DOX/MPLA(+) + anti-PD-L1), contrasting with M-DOX/MPLA $(+)$ without anti-PD-L1 for which tumor progression restarted 10 days after administration. ${ }^{76}$ In 2015 , Toomari and co-workers developed $\beta$-cyclodextrin ( $\beta$ CD) dendrimers based on CuAAC click chemistry. ${ }^{77}$ The encapsulation properties of $\beta-C D$ enable the loading of numerous MTX drugs which are better released over time in acidic $(\mathrm{pH}$ 3) rather than physiological $\mathrm{pH}$ conditions.

Other research has also been carried out on the use of NPs for encapsulating bioactive molecules to improve their pharmacokinetics. $\mathrm{pH}$-responsive polymeric NPs have been developed to embed Vorinostat. ${ }^{78}$ This molecule is known to treat cutaneous T-cell lymphoma by inhibiting the protein histone deacetylases (HDAC) overexpressed in cancer cells. The CuAAC reaction has been used to incorporate alkyne Vorinostat on azidomacromonomer before ring-opening metathesis copolymerization, a process for chaingrowth polymerization, using norbornene to drive the reaction. The authors thus developed a successful triggered-delivery system, which enhanced delivery in tumors thanks to the EPR effect and cellular internalization by endocytosis in an acid environment $(\mathrm{pH}<6)$.

In the same year, Yu et al. developed another biodegradable $\mathrm{pH}$-responsive polymeric NP encapsulating DOX. ${ }^{79}$ CuAAC was of interest in this study because it does not involve any protection/deprotection of the aliphatic polyester (polylactide, PLA) scaffolds typically used after polymerization. Alkyne-functionalized PLA was prepared for the incorporation of an azido-acid-labile Schiff base permitting controlled release of the DOX (pH 5.5). Nanoprecipitation, followed by addition of a biocompatible PEG surfactant enabled the formation of dispersible DOX-loaded NPs presenting maleimide functions available for the potential incorporation of targeting moieties via thiol-maleimide chemistry. In vitro studies on MCF-7 breast cancer cells showed a slightly larger decrease in cell viability compared with the free drug, suggesting promising results in vivo using passive tumor targeting. In another study, $\mathrm{pH}$-responsive micelles were synthesized for the co-delivery of chemotherapeutic drugs. Although the Pt(IV) prodrug was anchored using CuAAC, DOX was embedded in the core by physicochemical interactions. ${ }^{80}$ Internalized nanomicelles were then deteriorated in 
endosomes allowing DOX release $(\mathrm{pH}$ 5.5). The synergistic effect of both drugs released resulted in better cytotoxic activity $\left(\mathrm{IC}_{50}\right.$ up to $0.02 \mu \mathrm{M}$ at 72 hours post-incubation in $\mathrm{A} 357$ cells) compared with the free drug $\left(\mathrm{IC}_{50}\right.$ free DOX: up to $0.13 \mu \mathrm{M}$ at 72 hours post-incubation in A357 cells) or micelles with only one drug ( $\mathrm{IC}_{50}$ DOX-loaded micelles: up to $0.14 \mu \mathrm{M}$ at 72 hours in A357 cells). This system showed improved therapeutic efficacy of the drugs $\left(\mathrm{IC}_{50}\right.$ MSN-Pt: $25.95 \mu \mathrm{M}$ vs $\mathrm{IC}_{50}$ oxaliplatin: $\left.39.29 \mu \mathrm{M}\right)$ thanks to a higher cellular uptake $(80.11 \mathrm{ng}$ $\mathrm{Pt} / \mathrm{mg}$ protein at 4 hours post-incubation) than with the free drug $(0.69 \mathrm{ng} \mathrm{Pt} / \mathrm{mg}$ protein at 4 hours post-incubation) in HepG-2 cells.

In 2015, Noureddine and co-workers emphasized the benefits of developing the CuAAC reaction to obtain homogenous multi-functionalizable NP structures. This method resulted in structures carrying azide and alkyne groups, unlike conventional grafting methods for which uncontrolled loading and functionalization are often obtained. ${ }^{81}$ Click chemistry was used to develop light- and consequently $\mathrm{pH}$-triggered MSNs containing DOX. Under blue irradiation (at $365 \mathrm{~nm}$ ), the two moieties interact by energy transfer (FRET), inducing, upon protonation, dissociation of the acceptor, decluttering of the pores and finally DOX release.

In another study, Cai el al. demonstrated the utility of the CuAAC reaction for modifying side chain polymeric NPs with PEG-containing alkynes. ${ }^{82}$ Drug polymers (SS-CSPT) were covalently assembled with PEG using CuAAC, followed by self-assembly by nanoprecipitation to form particles. These thiol redox-responsive polymeric NPs provided an efficient controlled drug release system dependent on ester linker hydrolysis. They afforded cytotoxicity on HeLa cells that was significantly higher than irinotecan but similar to the SN$38\left(\mathrm{IC}_{50}: 19 \mathrm{nM}, 14 \mathrm{nM}\right.$ and $2800 \mathrm{nM}$, respectively) usually used in clinics, and efficient anticancer efficacy in vivo on MCF-7 cells (8.6\% vs $5.3 \%$ of irinotecan).

CuAAC has also been employed with $\mathrm{pH}$-responsive NMOFs to attach PEG to the surface to improve stability and cellular uptake. This also allowed the incorporation of fluorescent calceins for endocytosis studies or for therapeutic studies investigating anticancer drugs (e.g. dichloroacetic acids). ${ }^{83}$ The resulting PEGylated NPs (i.e. PEG550 and PEG2000) enhanced cellular uptake and therefore decreased cell viability at $0.75 \mathrm{mg} / \mathrm{mL}$ on HeLa cells compared with non-PEGylated NPs (50\% vs 125\%).

\section{Examples of thiol-ene chemistry}

In 2017, camptothecin (CPT) prodrug-functionalized MSNs obtained by thiol-ene click reactions demonstrated efficient release of the drug in acidic conditions $(\mathrm{pH} \mathrm{4.0)}$ thanks to the presence of cleavable silyl ether bonds, with tumor cell inhibition being similar to that achieved with the free drug ( $40 \%$ cell growth inhibition) (Figure) ${ }^{87}$
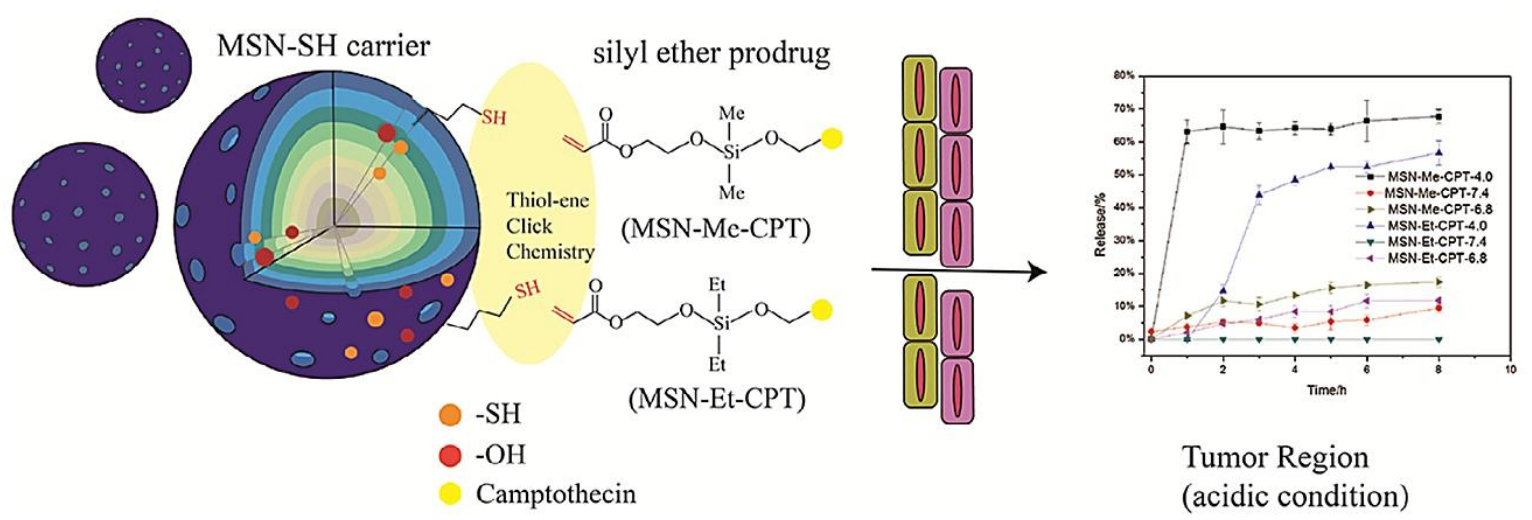
Figure 9. Illustration of an acid-responsive silyl prodrug being from an MSN-SH nanocarrier. Reprinted with permission from reference ${ }^{87}$. Copyright 2017 Elsevier.

\subsubsection{Complexation approaches}

\section{Examples of CuAAC}

Natural linear polysaccharides are widely used in the biomedical field for their biodegradability and biocompatibility properties. ${ }^{90}$ In 2015, Mai et al. performed CuAAC chemistry to synthesize a safe dendrimer structure based on amylose from potatoes. The positively-charged nanocarriers enabled the complexation of anionic plasmid DNA and in vitro studies confirmed efficient transfection (up to $70 \%$ transfection efficiency) without the use of a targeting ligand. ${ }^{84}$ The same authors achieved effective gene transfection in serum with high generation of PAMAM dendron nanocomplexes conjugated with dextran by CuAAC. $^{85}$

Another team implemented this strategy with clicked amylose dendrons for hemostatic action through thrombin complexation. ${ }^{86}$ The nanocomplexation of thrombin offered better stability than with native thrombin (activity retention percentage $>50 \%$ vs $<5 \%$ after 60 days, respectively). Moreover, it had the same hemostatic effect; firstly, in vitro with fibrinogen coagulation tests and in vivo on an artery hemorrhage rat model.

\section{Examples of thiol-ene chemistry}

The heterogeneity of tumors and increased drug resistance in cancer cells has made combination chemotherapy a common strategy to treat tumors. Cai et al. described dendrimer micelles containing two types of anti-tumor drugs: CDDP and PTX. ${ }^{88}$ The first was chelated by carboxyl groups incorporated by thiol-ene chemistry and inhibited DNA synthesis; while the second was only encapsulated and inhibited cell cycle mitosis. In vitro studies on ovarian cancer cells (SKOV-3) demonstrated a decrease of $\mathrm{IC}_{50}$ by combining a 2:1 ratio of CDDP/PTX $\left(\mathrm{IC}_{50}: 39 / 19.5 \mathrm{ng} / \mathrm{mL}\right)$ compared with free CDDP and PTX $\left(\mathrm{IC}_{50}\right.$ : 1354 and $32 \mathrm{ng} / \mathrm{mL}$, respectively). In vivo, this synergistic effect significantly improved tumor growth inhibition (relative tumor volume $\left(\mathrm{RTV}_{\mathrm{CDDP} / \mathrm{PTX}}\right)=2$ vs $\mathrm{RTV}_{\mathrm{CDDP}}=4$ and $\mathrm{RTV}_{\mathrm{PTX}}=11$ ) and medium survival time (40 days) compared with just PTX (23 days) or CDDP (34 days) for the same drug dosage (6 mg/kg CDDP and $3 \mathrm{mg} / \mathrm{kg}$ PTX).

Thiol-ene chemistry has also been investigated to design MSNs containing 1,2-bidentate carboxyl groups for efficient anticancer drug chelation of dichloro(1,2diaminocyclohexane)platinum(II) (DACH-Pt). ${ }^{89}$

\subsection{Passive targeting with surface-functionalized NPs}

As well as encapsulation and complexation, some articles concern the use of click chemistry for: (i) conjugating anticancer drugs and chemotherapeutic agents on the surface of NPs; (ii) adding stimuli-responsive linkers; and (iii) incorporating reporter molecules or contrast agents onto the surface of NPs (Table 2).

\subsubsection{Surface functionalization with anticancer drugs}


In general, passive targeting NPs are subsequently delivered intratumorally after EPR accumulation and drugs are specifically released by $\mathrm{pH}$ or enzyme-responsive linkages. Nanomaterials with enzyme-responsive linkages are ideal for selective delivery of drugs into tumor cells because of their up-regulation and cellular actions in tumor tissue. Tumorassociated enzyme matrix metalloproteinase (MMP) is an enzyme of interest that is overexpressed in cancer cells and able to cleave peptide sequences selectively in the TME for controlled drug delivery. ${ }^{91}$ The specific peptide substrates are usually deposited onto nanoscale vehicles and act as linkers for the conjugation of antitumor drugs.

Table 2. General overview of passive targeting NPs using click chemistry to incorporate drugs on the surface, from 2014 to nowadays.

\begin{tabular}{|c|c|c|c|c|c|c|}
\hline $\begin{array}{l}\text { Type of click } \\
\text { chemistry and use } \\
\text { (grafted click function) }\end{array}$ & $\begin{array}{l}\text { Type of NPs } \\
\text { (grafted click } \\
\text { function) }\end{array}$ & Drug loading & $\begin{array}{l}\text { Release } \\
\text { mechanism }\end{array}$ & Effects & $\begin{array}{l}\text { In vitro/In vivo } \\
\text { studies }\end{array}$ & Refs. \\
\hline $\begin{array}{l}\text { CuAAC, incorporation of } \\
\text { bile acid (Alkyne) }\end{array}$ & Dendrimers $\left(\mathbf{N}_{\mathbf{3}}\right)$ & Bile acid & n.d & Cytotoxicity & yes/no & 23 \\
\hline $\begin{array}{l}\text { CuAAC, incorporation of } \\
\text { MMP-2 peptide (Alkyne) } \\
\text { and formation of } \\
\text { nanoclusters }\end{array}$ & AuNPs $\left(\mathbf{N}_{3}\right)$ & DOX & $\begin{array}{l}\text { Enzymatic } \\
\text { cleavage (MMP } \\
\text { cleavage) }\end{array}$ & Anticancer activity & no/yes & 27 \\
\hline $\begin{array}{l}\text { CuAAC, formation of } \\
\text { nanoclusters between } \\
\text { DOX-AuNPs and MMP- } \\
\text { QDs }\end{array}$ & $\begin{array}{l}\text { AuNPs }\left(\mathbf{N}_{\mathbf{3}}\right) \text { and } \\
\text { QDs (Alkyne) }\end{array}$ & DOX & $\begin{array}{l}\text { Enzymatic } \\
\text { cleavage } \\
\text { (MMP cleavage) }\end{array}$ & $\begin{array}{l}\text { Cytotoxicity and } \\
\text { anticancer activity }\end{array}$ & yes/yes & 92 \\
\hline $\begin{array}{l}\text { CuAAC, incorporation of } \\
\text { gemcitabine }\end{array}$ & $\begin{array}{l}\text { Dendrimers } \\
\text { (Alkyne) }\end{array}$ & Gemcitabine $\left(\mathbf{N}_{\mathbf{3}}\right)$ & $\begin{array}{l}\text { Enzymatic } \\
\text { cleavage } \\
\text { (Cathepsin- } \\
\text { sensitive linker) }\end{array}$ & $\begin{array}{l}\text { Cytotoxicity and } \\
\text { anticancer activity }\end{array}$ & yes/yes & 93 \\
\hline $\begin{array}{l}\text { CuAAC, incorporation of } \\
\text { DOX on the surface }\end{array}$ & $\begin{array}{l}\text { Dendrons } \\
\text { (Alkyne) }\end{array}$ & $\operatorname{DOX}\left(\mathbf{N}_{\mathbf{3}}\right)$ & $\begin{array}{l}\text { Enzymatic } \\
\text { cleavage } \\
\text { (Cathepsin- } \\
\text { sensitive linker) }\end{array}$ & $\begin{array}{l}\text { Cytotoxicity and } \\
\text { anticancer activity }\end{array}$ & yes/yes & 94 \\
\hline $\begin{array}{l}\text { CuAAC, incorporation of } \\
\text { the neuroprotective drug } \\
\text { (minocycline) }\end{array}$ & $\begin{array}{l}\text { Dendrimers } \\
\text { (Alkyne) }\end{array}$ & Minocycline $\left(\mathbf{N}_{\mathbf{3}}\right)$ & $\begin{array}{l}\text { Ester linker } \\
\text { hydrolysis }\end{array}$ & $\begin{array}{l}\text { Treatment of } \\
\text { neuroinflammation }\end{array}$ & yes/yes & 95 \\
\hline $\begin{array}{l}\text { CuAAC, incorporation of } \\
\text { oligonucleotides (Alkyne) } \\
\text { during nanogel formation }\end{array}$ & Nanogels $\left(\mathbf{N}_{3}\right)$ & DOX & $\begin{array}{l}\text { Hybridization } \\
\text { reaction and } \\
\text { oligonucleotide } \\
\text { degradation }\end{array}$ & Cytotoxicity & yes/no & 96 \\
\hline $\begin{array}{l}\text { CuAAC, incorporation of } \\
\text { HDACi (Alkyne) }\end{array}$ & Polymeric $\left(\mathbf{N}_{\mathbf{3}}\right)$ & HDACi & $\begin{array}{l}\text { Acid-responsive } \\
\text { release }\end{array}$ & $\begin{array}{l}\text { Cytotoxicity and } \\
\text { anticancer activity }\end{array}$ & yes/yes & 97,98 \\
\hline $\begin{array}{l}\text { SPAAC, Incorporation of } \\
\text { immune cells and } \\
\text { possibility of incorporating } \\
\text { drugs }\left(\mathbf{N}_{\mathbf{3}}\right)\end{array}$ & $\begin{array}{l}\text { Dendrimers } \\
\text { (DBCO) }\end{array}$ & $\begin{array}{l}\text { Immune cells } \\
\text { (RAW 264.7) }\end{array}$ & n.d & n.d & yes/no & 99 \\
\hline
\end{tabular}

n.d: not determined, $\mathrm{N}_{3}$ : azide, DBCO: dibenzocyclooctyne, DOX: doxorubicin, HDACi: histone deacetylase inhibitor, MMP: matrix metalloproteinase, AuNPs: gold nanoparticles.

"Effects" indicate the NP effects on cancer cells in vitro and/or in vivo.

"In vitro/in vivo studies" indicate whether in vitro or in vivo tests were performed (yes) or not (no) in the article.

\section{Examples of CuAAC}

In 2017, bile acid was efficiently grafted by CuAAC click reactions on dendrimers and demonstrated higher cytotoxicity towards glioma cells with second generation dendrimers (up to $99.31 \%$ inhibition at $50 \mu \mathrm{M} ; \mathrm{IC}_{50}: 10.68 \mu \mathrm{M}$ ) compared with first generation dendrimers (up to $94.1 \%$ inhibition at $\left.50 \mu \mathrm{M} ; \mathrm{IC}_{50}: 14.86 \mu \mathrm{M}\right){ }^{23}$ 
Mao et al. recently used click chemistry to form nanoclusters (DOX@AuNCs) between azidemodified AuNPs bearing DOX (DOX/N $\left.\mathrm{N}_{3} @ A u N P s\right)$ and MMP-2 cleavable peptides with an alkyne group at each end (Figure). ${ }^{27}$ Intravenous injection of DOX@AuNCs in vivo demonstrated efficient tumor suppression 28 days after injection on human lung carcinoma tumors compared with DOX@AuNPs and free DOX (tumor volume for DOX@AuNCs, DOX@AuNPs and DOX: $50 \mathrm{~mm}^{3}, 250 \mathrm{~mm}^{3}$ and $280 \mathrm{~mm}^{3}$, respectively).
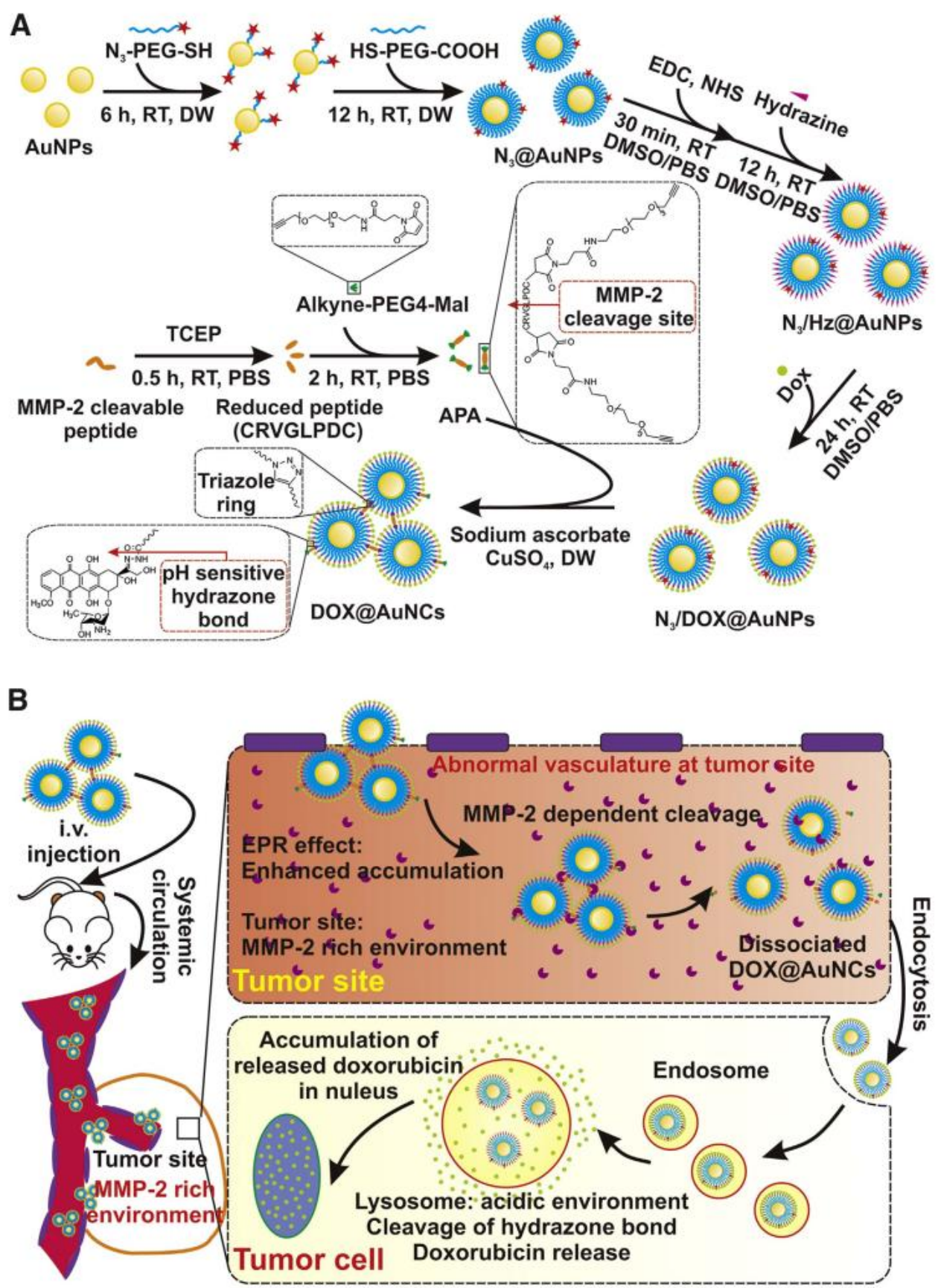

Figure 10. Fabrication and anticipated in vivo behaviors of DOX@AuNCs. (A) Fabrication of DOX@AuNCs by copper(I)-catalyzed azide-alkyne cycloaddition of DOX/N3@AuNPs and APA. Azido groups were immobilized on AuNPs using N3-PEG-SH and DOX was conjugated to AuNPs by a pH-sensitive hydrazone bond. (B) Intravenous injection of DOX@AuNCs in tumor-bearing mouse. The DOX@AuNCs could target tumor sites by EPR effect. Following by entering tumor sites, the APA between AuNPs are digested by abundant MMP-2, and the digested particles are endocytosed by tumor cell, and finally release DOX in low pH lysosome. The released DOX could target nucleus and induce cell apoptosis thus suppress tumor growth. Reprinted with permission from re $^{27}$. Copyright 2018 Elsevier. 
This anticancer effect was evaluated in vivo with CT images of tumors after accumulation of Gd-functionalized nanoclusters (Gd@AuNCs). Due to their size (250-300 nm), DOX@AuNCs were 150 times more retained in the tumor site compared with DOX@AuNPs alone (20-30 $\mathrm{nm}$ ). This suggests preferential accumulation of DOX@AuNCs at the tumor site due to EPR effects facilitating the release of $\mathrm{DOX}(\mathrm{pH}<5.5)$ after nanocluster dissociation by MMP cleavage.

Similarly, Kim and colleagues described clicked DOX nanoclusters composed of AuNPs and QDs for imaging and therapy. ${ }^{92}$ They also used a MMP-cleavable peptide to cleave the particles selectively and release DOX onto AuNPs under reducing conditions using glutathione. They highlighted the advantage of using passively targeted NPs in vivo for a prolonged anticancer effect. After 15 days, the nanoclusters demonstrated greater tumor regression compared with DOX alone (relative tumor volume: $0.8 \%$ at 15 days to $0.4 \%$ at 28 days $v s 0.8 \%$ at 28 days).

A similar study also used CuAAC reactions to synthesize PEGylated dendrimer-gemcitabine (dendrimer-GEM) conjugates to control the release of gemcitabine by cathepsin $\mathrm{B},{ }^{93} \mathrm{a}$ cystein protease known to be involved in cancer and metastatic cell progression and to activate MMPs. ${ }^{100}$ They demonstrated higher tumor growth inhibition of $89.92 \%$ in vivo on 4T1 murine breast cancer cells compared with free GEM, which gave only $44.59 \%$ inhibition. This was due to the local concentration of dendrimer-GEM in tumors under the EPR effect and enzyme-responsive drug release.

The same observations were made in 2017 on PEGylated dendrons conjugated with DOX (mPEGylated dendron-GFLG-DOX) via cathepsin-sensitive linkers using CuAAC chemistry. ${ }^{94}$ An efficient antitumoral effect on breast cancer was observed compared with the free drug with much higher tumor growth inhibition (80.3\% vs 57.3\%, respectively).

Sharma and co-workers demonstrated the ability of fluorescent PAMAM dendrimers known to cross the impaired BBB for the targeting of specific neuroinflammation mediators ${ }^{95}$. A neuroprotective drug (i.e. minocycline) was introduced using CuAAC (D-mino) and selectively released in acidic conditions ( $\mathrm{pH} 5.5$ ) by cleaving the amide bond by esterase. Dmino showed superior anti-inflammatory and antioxidant actions in microglial cells by significantly inhibiting inflammatory cytokine tumor necrosis factor $\alpha$ (TNF- $\alpha$ ) and nitric oxide (NO) production compared with free minocycline at the same $500 \mu \mathrm{M}$ concentration (TNF- $\alpha$ released: $566.5 \mathrm{pg} / \mathrm{mL}$ vs $1150 \mathrm{pg} / \mathrm{mL}$ (no reduction); NO released: $0.12 \mu \mathrm{M}$ vs $2.92 \mu \mathrm{M}$ ).

In another report, CuAAC enabled controlled release of DOX after hybridization with a complementary ODN duplex-modified hydrogel and nuclease degradation of the oligonucleotides. ${ }^{96}$ The reversible hybridization platform demonstrated a $10 \%$ decrease in cell viability compared with the same platform and unloaded DOX nanogels.

CuAAC has also been used to introduce histone deacetylase inhibitor (HDACi) acidresponsive prodrug on polymeric NPs. ${ }^{97,98}$ The NPs exhibited a positive antitumor effect in vivo on AK7 murine mesothelioma cells with $80 \%$ tumor weight regression thanks to the passive targeting induced by NPs.

\section{Examples of SPAAC}

SPAAC chemistry has also been investigated for the design of cell-dendrimer hybrids. ${ }^{99}$ SPAAC occurs between $\mathrm{N}_{3}$-modified immune cells (RAW264.7) obtained by metabolic glycol- 
engineering and clickable DBCO-dendrimers. More importantly, the newly functionalized RAW cells preserve their viability and intracellular pathway, suggesting potential future applications for these cell-NP hybrids as drug delivery systems. These dendrimers also offer the possibility of including other molecules of interest as drugs and imaging agents.

\subsubsection{Surface functionalization with imaging agents}

The emergence of nanomedicines has opened new perspectives for the use of imaging probes for both molecular and cellular imaging. ${ }^{101}$ Click chemistry developments in recent years have enabled the introduction of contrast agents for imaging, as well as functionalization with a variety of molecules. ${ }^{50}$ In the articles of this study, several nanomaterials are combined with multiple imaging methods to improve diagnostic efficiency. Single-photon emission computed tomography (SPECT), computed tomography (CT), magnetic resonance imaging (MRI), positron emission tomography (PET) and optical imaging (fluorescence) are the main techniques used for multimodal cancer imaging. ${ }^{102-104}$ In particular, light in the near infrared (NIRF: $650-900 \mathrm{~nm}$ ) is frequently combined with PET or MR functionalities because it allows deeper tissue penetration thus improving in vivo detection of tumors (Table 3 ).

Table 3. General overview of passive targeting NPs using click chemistry to incorporate imaging agents on the surface, from 2014 to nowadays.

\begin{tabular}{|c|c|c|c|c|c|}
\hline $\begin{array}{l}\text { Type of click } \\
\text { chemistry and use } \\
\text { (grafted click } \\
\text { function) }\end{array}$ & $\begin{array}{l}\text { Type of NPs } \\
\text { (grafted click } \\
\text { function) }\end{array}$ & $\begin{array}{l}\text { Imaging agent } \\
\text { (grafted click function) }\end{array}$ & $\begin{array}{l}\text { Type of } \\
\text { imaging }\end{array}$ & $\begin{array}{l}\text { In vitro/In vivo } \\
\text { studies }\end{array}$ & Refs. \\
\hline $\begin{array}{l}\text { CuAAC, } \\
\text { incorporation of NIR } \\
\text { dye }\end{array}$ & $\mathrm{Fe}_{3} \mathrm{O}_{4}\left(\mathbf{N}_{3}\right)$ & IR675 (Alkyne) & MR, NIRF & yes/yes & 105 \\
\hline $\begin{array}{l}\text { CuAAC, } \\
\text { incorporation of } \\
\text { dyes }\end{array}$ & $\begin{array}{l}\text { Polymeric } \\
\text { (Alkyne) }\end{array}$ & $\begin{array}{l}\text { Dyes (Cy3, ATTO-740, } \\
\text { coumarin } 343)\left(\mathbf{N}_{3}\right)\end{array}$ & Optical & yes/no & 106 \\
\hline $\begin{array}{l}\text { CuAAC, } \\
\text { incorporation of } \\
\text { metalloporphyrins }\end{array}$ & $\mathrm{Fe}_{3} \mathrm{O}_{4}\left(\mathbf{N}_{3}\right)$ & $\begin{array}{l}\text { Metalloporphyrin } \\
\text { complexed with Zn } \\
\text { (Alkyne) }\end{array}$ & $\begin{array}{l}\text { Photonic, } \\
\text { optical }\end{array}$ & no/no & 107 \\
\hline $\begin{array}{l}\text { SPAAC, } \\
\text { incorporation of } \\
\text { imaging probes }\end{array}$ & $\begin{array}{l}\text { Glycol chitosan } \\
\left(\mathbf{N}_{3}\right)\end{array}$ & $\begin{array}{l}{ }^{64} \mathrm{Cu}-\mathrm{DOTA}, \mathrm{Cy} 5.5-\mathrm{MMP} \\
\text { (DBCO) }\end{array}$ & PET, NIRF & yes/yes & 108 \\
\hline $\begin{array}{l}\text { SPAAC, } \\
\text { incorporation of } \\
\text { chelated }{ }^{89} \mathrm{Zr}\end{array}$ & $\begin{array}{l}\text { Liposomes } \\
\text { (DBCO) }\end{array}$ & ${ }^{89} \mathrm{Zr}-\mathrm{CLL}\left(\mathbf{N}_{3}\right)$, Cy5 & PET, NIRF & no/yes & 109 \\
\hline $\begin{array}{l}\text { SPAAC, } \\
\text { incorporation of } \\
\text { imaging probes }\end{array}$ & AuNPs (DBCO) & ${ }^{125} \mathrm{I}\left(\mathbf{N}_{3}\right)$ & PET & no/yes & 110 \\
\hline $\begin{array}{l}\text { SPAAC, } \\
\text { incorporation of IR } \\
\text { dye }\end{array}$ & $\begin{array}{l}\text { Gold nanorods } \\
\left(\mathbf{N}_{3}\right)\end{array}$ & IRDye 800CW (DBCO) & Optical & no/no & 31 \\
\hline
\end{tabular}

$\mathrm{N}_{3}$ : azide, DBCO: dibenzocyclooctyne, $\mathrm{Fe}_{3} \mathrm{O}_{4}$ : iron oxide nanoparticles, AuNPs: gold nanoparticles, MMP: matrix metalloproteinase, MR: magnetic resonance, NIR: near infrared, PET: positron emission tomography.

"In vitro/in vivo studies" indicate whether in vitro or in vivo tests were performed (yes) or not (no) in the article.

\section{Examples of CuAAC}


This type of click chemistry has been explored for the efficient preparation of bimodal NPs combining by MR and NIRF imaging to improve in vivo imaging. ${ }^{105}$

The use of CuAAC for the post-functionalization of copolymeric NPs with diverse organic dyes (Cy3, ATTO-740 or coumarin 343) allows fluorescence signal location. ${ }^{106}$ The mild conditions of copper-free click reactions enhance the stability of dye-labelled SPION, by reducing aggregation and allowing in vivo detection, which was not possible with the conjugation processes used previously.

Hollingsworth et al. synthesized metalloporphyrin-modified superparamagnetic silica-coated $\mathrm{Fe}_{3} \mathrm{O}_{4} \mathrm{NPs}$ by $\mathrm{CuAAC}$ for photonic and optical applications. ${ }^{107}$

\section{Examples of SPAAC}

Bimodal nanotherapeutics combining two types of imaging, such as PET/NIRF, have been demonstrated. Lee and co-workers developed glycol chitosan NPs (CNPs) as imaging probes combining PET and NIRF. ${ }^{108}$ SPAAC was used for both functionalizing azide CNPs with ${ }^{64} \mathrm{Cu}$ through radiolabeling of DOTA-Lys-PEG $-\mathrm{DBCO}$ and for Cy5.5 labeling with MMP activable peptide (Figure11). The latter was conjugated with a dark quencher (BHQ3) and released in the presence of specific MMPs (MMP-2, 13 and 9 particularly) to enhance fluorescence signals. The bimodal imaging probe thus formed was able to provide information on the biodistribution and accumulation of NPs in the tumor site, and on the activity of the MMP biological marker overexpressed in cancer cells. Thanks to dual-modality imaging, NPs can provide high sensitivity associated with a good depth of penetration via PET as well as specific molecular detection through optical imaging.

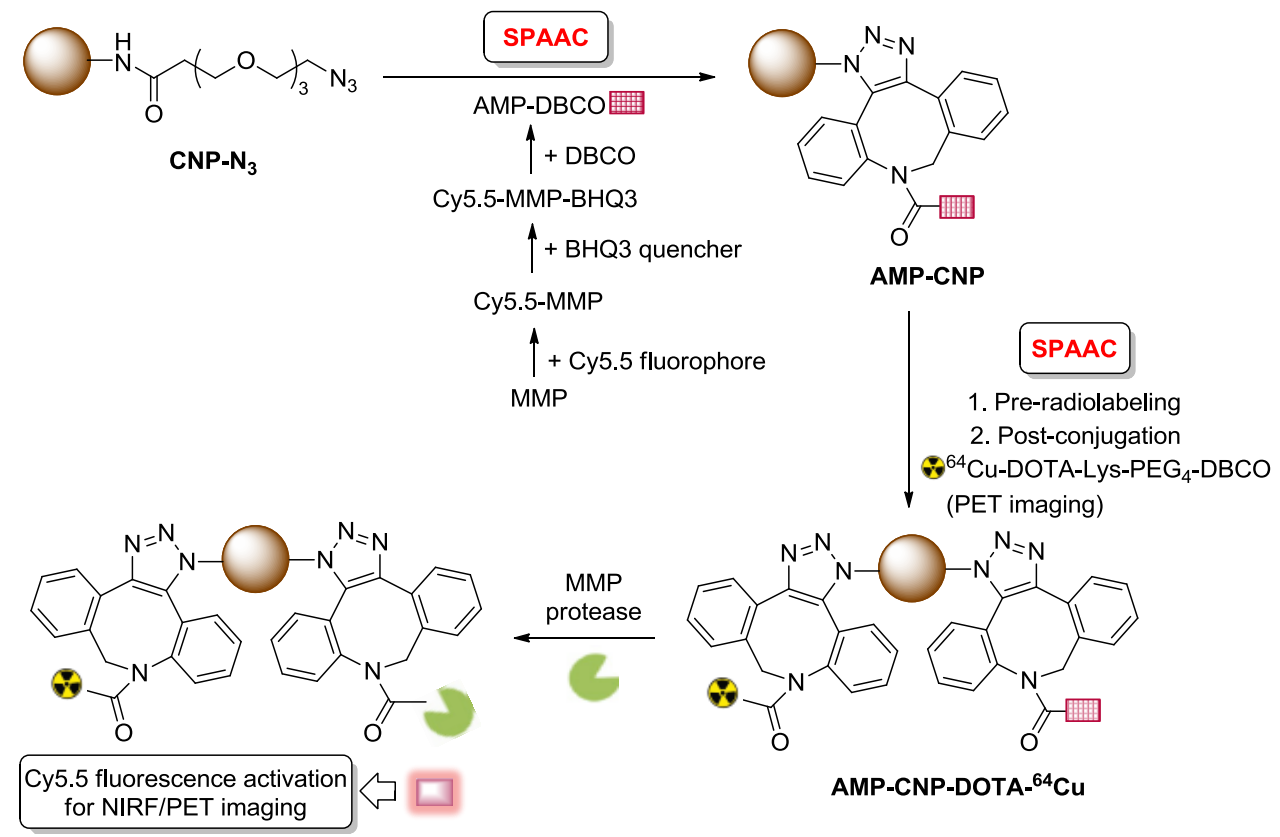

Figure 11. Schematic representation of MMP-sensitive NPs labeled using copper free click chemistry (SPAAC) with AMP-DBCO (comprising MMP-specific peptide, Cy5.5 NIRF dye and BHQ-3 (dark quencher) and 64CuDOTA-Lys-PEG4-DBCO. CNP: chitosan nanoparticles, MMP: matrix metalloproteinase, AMP: Activatable MMPspecific peptide probe, Lys: Lysine, DOTA: 1,4,7,10-Tetraazacyclododecane-1,4,7,10-tetraacetic acid, NIRF: near infrared, PET: positron emission tomography. Figure modified from ref ${ }^{108}$.

In 2015, SPAAC was employed to link DBCO-modified AuNPs with ${ }^{125}$-labeled azide giving an excellent radiochemical yield (> 95\%) for potential use an as imaging probe. ${ }^{110}$ SPAAC 
has also been used to develop ${ }^{89} \mathrm{Zr}$-labeled liposomal NPs ( ${ }^{89} \mathrm{Zr}$-CLL) using in vivo dual PET/ NIRF imaging. ${ }^{109}$

In another study, silica-coated gold nanorods combining fluorescence and surface plasmon resonance (SPR) showed attractive fluorescent properties for future biomedical applications. ${ }^{31}$

The benefits of passive targeting NPs obtained by click chemistry enable good therapeutic and imaging efficacy. However, a recent meta-analysis by Wilhelm et al. found that a median of only $0.7 \%$ ID of NPs are accumulated in tumors after injection. ${ }^{16}$ In fact, numerous studies have demonstrated that the EPR effect is highly dependent on TME heterogeneity between models and individuals, and reliant on the physical and chemical properties of the NPs. ${ }^{74,111,112}$

Various studies have thus tried to improve tumor accumulation and biodistribution by taking into account these parameters and TME heterogeneity. ${ }^{69,113,114}$ The effects of size, shape and surface properties have been investigated to design smart and effective therapeutic NPs. ${ }^{115}$ For example, rod-shaped NPs induce a higher cell uptake than cubes, cylinders or even spheres. Positively-charged NPs are also preferred because of a possible electrostatic interaction with negatively charged cell-membranes. ${ }^{116}$

Another promising strategy to overcome TME heterogeneity consists in developing active targeting NPs. Unlike passive targeting, active targeting relies on the conjugation of tumorspecific biomarkers onto nanoparticles that are generally overexpressed in the TME. An important question remains: could the NPs be considered as a "miracle recipe" if they could be addressed specifically by grafting the targeting molecule using click chemistry?

\section{Influence of active targeting using click chemistry on tumor accumulation and cellular uptake: non-targeting vs targeting NPs}

It has been widely reported that active targeting can facilitate specific uptake in cancer cells, although very few active targeting nanomedicines are under clinical trial (e.g. MCC-465, SGT-53, CALAA-01 in phase I and BIND-014 in phase II). ${ }^{117}$ Many different kinds of targeting moieties overexpressed on tumor cells can be introduced by click chemistry for specific cellular interaction. Click chemistry is therefore considered to be a carefully engineered approach for the development of new molecular delivery systems and represents $38 \%$ of the articles listed in this review. The molecules most investigated for targeting are folates, carbohydrates, cell-binding peptides (e.g. integrin ligands and cell-penetrating peptides), proteins, mAbs and oligonucleotides. Many studies describing click chemistry for this purpose highlight efficient preparations of NP-conjugates to target cancer cells and compare the effects of active targeting NPs on cancer cells (Table 4). 
Table 4. General overview of articles comparing active targeting NPs and passive targeting NPs functionalized by click chemistry, from 2014 to nowadays

\begin{tabular}{|c|c|c|c|c|c|c|c|}
\hline & $\begin{array}{l}\text { Type of click chemistry and } \\
\text { use (grafted click function) }\end{array}$ & $\begin{array}{l}\text { Targeting } \\
\text { molecule }\end{array}$ & Target & $\begin{array}{l}\text { Type of NPs } \\
\text { (grafted click } \\
\text { function) }\end{array}$ & Drug loading & $\begin{array}{l}\text { Effects compared to passive targeting } \\
\text { NPs }\end{array}$ & Refs. \\
\hline \multirow{8}{*}{ 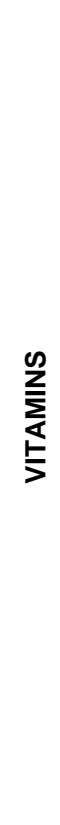 } & $\begin{array}{l}\text { CuAAC, incorporation of folic } \\
\text { acid }\end{array}$ & Folic acid $\left(\mathbf{N}_{3}\right)$ & $\begin{array}{l}\text { Folate } \\
\text { receptor }\end{array}$ & $\begin{array}{l}\text { Cerium oxide } \\
\text { (Alkyne) }\end{array}$ & $\begin{array}{l}\text { DOX and } \\
\text { Ganetespib }\end{array}$ & Higher cellular uptake & 118 \\
\hline & $\begin{array}{l}\text { CuAAC, incorporation of folic } \\
\text { acid }\end{array}$ & $\begin{array}{l}\text { Folic acid } \\
\text { (Alkyne) }\end{array}$ & $\begin{array}{l}\text { Folate } \\
\text { receptor }\end{array}$ & Polymeric $\left(\mathbf{N}_{3}\right)$ & MTX & $\begin{array}{l}\text { 17\% higher cellular uptake } \\
\text { 3-fold higher cytotoxicity }\end{array}$ & 119 \\
\hline & $\begin{array}{l}\text { CuAAC, incorporation of folic } \\
\text { acid }\end{array}$ & Folic acid $\left(\mathbf{N}_{3}\right)$ & $\begin{array}{l}\text { Folate } \\
\text { receptor }\end{array}$ & Micelles (Alkyne) & DOX & 2.2-fold higher cellular uptake & 120 \\
\hline & $\begin{array}{l}\text { CuAAC, incorporation of folic } \\
\text { acid }\end{array}$ & $\begin{array}{l}\text { Folic acid } \\
\text { (Alkyne) }\end{array}$ & $\begin{array}{l}\text { Folate } \\
\text { receptor }\end{array}$ & $\mathrm{MSNs}\left(\mathbf{N}_{3}\right)$ & DOX & $7 \%$ higher cytotoxicity & 38 \\
\hline & $\begin{array}{l}\text { CuAAC, incorporation of PEG- } \\
\text { biotin on PLGA polymer }\end{array}$ & $\operatorname{Biotin}\left(\mathbf{N}_{3}\right)$ & Biotin receptor & Polymeric (Alkyne) & DOX & $\begin{array}{l}\text { 2-fold higher cellular uptake } \\
\text { 1.4-fold higher cytotoxicity } \\
\text { 2.2-fold higher tumor uptake } \\
\text { 2.3-fold higher antitumor activity }\end{array}$ & 121 \\
\hline & $\begin{array}{l}\text { SPAAC, incorporation of folic } \\
\text { acid }\end{array}$ & Folic acid (BCN) & $\begin{array}{l}\text { Folate } \\
\text { receptor }\end{array}$ & AuNPs $\left(\mathbf{N}_{3}\right)$ & & Up to 4.3-fold higher cellular binding & 122 \\
\hline & $\begin{array}{l}\text { SPAAC, incorporation of folic } \\
\text { acid }\end{array}$ & Folic acid (BCN) & $\begin{array}{l}\text { Folate } \\
\text { receptor }\end{array}$ & Polymeric $\left(\mathbf{N}_{3}\right)$ & & 2.5-fold higher cellular binding & 123 \\
\hline & $\begin{array}{l}\text { IEDDA, incorporation of folic } \\
\text { acid }\end{array}$ & Folic acid (Tz) & $\begin{array}{l}\text { Folate } \\
\text { receptor }\end{array}$ & $\begin{array}{l}\text { MSNs } \\
\text { (Norbornene) }\end{array}$ & Actinomycin D & Higher cellular uptake & 37 \\
\hline
\end{tabular}


Table 4: to be continued

\begin{tabular}{|c|c|c|c|c|c|c|c|}
\hline & $\begin{array}{l}\text { Type of click chemistry and } \\
\text { use (grafted click function) }\end{array}$ & Targeting molecule & Target & $\begin{array}{l}\text { Type of NPs } \\
\text { (grafted click } \\
\text { function) }\end{array}$ & Drug loading & Effects compared to passive targeting NPs & Refs. \\
\hline 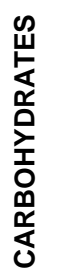 & $\begin{array}{l}\text { CuAAC, incorporation of } \mathrm{HA} \\
\text { polymer }\end{array}$ & $\begin{array}{l}\text { Hyaluronic acid } \\
\text { (Alkyne) }\end{array}$ & CD44 receptor & Lipidic $\left(\mathbf{N}_{3}\right)$ & siRNA & $\begin{array}{l}\text { 1.8-fold higher cellular uptake } \\
10 \% \text { higher cytotoxicity } \\
15 \% \text { higher gene-silencing in vitro } \\
2 \text {-fold higher tumor uptake and antitumor activity } \\
30 \% \text { higher gene-silencing in vivo }\end{array}$ & 124 \\
\hline \multirow{2}{*}{ 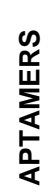 } & SPAAC, incorporation of DNA & AS14111 (DBCO) & Nucleolin receptor & NMOFs $\left(\mathbf{N}_{3}\right)$ & DOX & $10 \%$ higher cytotoxicity & 125 \\
\hline & SPAAC, incorporation of DNA & DNA (DBCO) & $\begin{array}{l}\text { Class A scavenger } \\
\text { receptor }\end{array}$ & $\mathrm{NMOFs}\left(\mathbf{N}_{3}\right)$ & & 5-fold higher cellular uptake & 126 \\
\hline \multirow{7}{*}{ 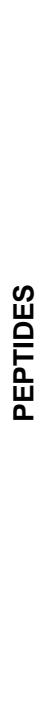 } & $\begin{array}{l}\text { CuAAC, incorporation of iRGD, } \\
\text { RGDS and poly(glutamic acid) }\end{array}$ & $\begin{array}{l}\text { iRGD, RGDS and } \\
\text { poly(glutamic acid) } \\
\left(\mathbf{N}_{3}\right)\end{array}$ & $\begin{array}{l}\text { Integrin } \beta_{3} \text { and } \\
\gamma \text {-glutamyl } \\
\text { transpeptidase }\end{array}$ & PSi (Alkyne) & & Higher cellular uptake & 127 \\
\hline & CuAAC, incorporation of RGE & $\operatorname{RGE}\left(\mathbf{N}_{3}\right)$ & Neuropilin-1 & $\begin{array}{l}\text { Exosomes (Alkyne) } \\
\text { embedding SPION } \\
\text { NPs }\end{array}$ & Curcumin & $\begin{array}{l}60 \% \text { higher cellular targeting } \\
20 \% \text { higher cytotoxicity } \\
3 \text {-fold higher tumor targeting } \\
1.34 \text {-fold higher antitumor activity }\end{array}$ & 128 \\
\hline & CuAAC, incorporation of RGD & RGD (Alkyne) & Integrin $\beta_{3}$ & Micelles $\left(\mathbf{N}_{3}\right)$ & PTX & $\begin{array}{l}\text { 3-fold higher cellular uptake } \\
\text { 14-fold higher cytotoxicity } \\
\text { Higher antitumor activity }\end{array}$ & 129 \\
\hline & CuAAC, incorporation of RGD & $\operatorname{RGD}\left(\mathbf{N}_{3}\right)$ & Integrin $\beta_{3}$ & $\mathrm{Fe}_{3} \mathrm{O}_{4}($ Alkyne $)$ & & 1.4-fold higher cellular binding & 130 \\
\hline & CuAAC, incorporation of $\mathrm{CRGD}$ & $\operatorname{cRGD}\left(\mathbf{N}_{3}\right)$ & Integrin $\beta_{3}$ & NDs (Alkyne) & & 12-fold higher cellular binding & 131 \\
\hline & $\begin{array}{l}\text { CuAAC, incorporation of CRGD } \\
\text { and BODIPY }\end{array}$ & cRGD (Alkyne) & Integrin $\beta_{3}$ & $\mathrm{Fe}_{3} \mathrm{O}_{4}\left(\mathbf{N}_{3}\right)$ & & Higher cellular uptake & 132 \\
\hline & $\begin{array}{l}\text { CuAAC, incorporation of F3- } \\
\text { peptide }\end{array}$ & F3-peptide (Alkyne) & Nucleolin & Polymeric $\left(\mathbf{N}_{3}\right)$ & DOX & 10 to 20 -fold higher cellular uptake & 133 \\
\hline
\end{tabular}


Table 4: to be continued

\begin{tabular}{|c|c|c|c|c|c|c|c|}
\hline & $\begin{array}{l}\text { Type of click chemistry and use } \\
\text { (grafted click function) }\end{array}$ & Targeting molecule & Target & $\begin{array}{l}\text { Type of NPs } \\
\text { (grafted click } \\
\text { function) }\end{array}$ & Drug loading & $\begin{array}{l}\text { Effects compared to passive targeting } \\
\text { NPs }\end{array}$ & Refs. \\
\hline \multirow{4}{*}{$\begin{array}{l}\text { 㟔 } \\
\text { 点 } \\
\text { 㟧 }\end{array}$} & $\begin{array}{l}\text { SPAAC, incorporation of iRGD and } \\
\text { DOTA }\end{array}$ & $\operatorname{iRGD}\left(\mathbf{N}_{3}\right)$ & Integrin $\beta_{3}$ & PSi (DBCO) & Sorafenib & $\begin{array}{l}\text { No difference in cytotoxicity } \\
1.63 \text {-fold higher tumor accumulation } \\
\text { No difference in tumor growth inhibition }\end{array}$ & 134 \\
\hline & SPAAC, incorporation of RGDS & iRGD and RGDS $\left(\mathbf{N}_{3}\right)$ & Integrin $\beta_{3}$ & $\mathrm{PSi}(\mathrm{BCN})$ & Sorafenib & $\begin{array}{l}20 \% \text { higher cellular uptake for RGDS } \\
15 \% \text { higher cytotoxicity for RGDS }\end{array}$ & 135 \\
\hline & SPAAC, incorporation of GLA & GLA peptide $\left(\mathbf{N}_{3}\right)$ & PSMA receptor & $\begin{array}{l}\text { Dendrimers } \\
\text { (Cyclooctyne) }\end{array}$ & MTX & 8-fold higher cellular uptake & 136 \\
\hline & SPAAC, incorporation of LHRH & LHRH peptide (DBCO) & LHRH receptor & Polymeric $\left(\mathbf{N}_{3}\right)$ & p53 & Up to $70 \%$ higher cytotoxicity & 137 \\
\hline \multirow{2}{*}{ 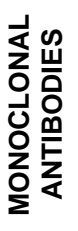 } & CuAAC, incorporation of anti-HER-2 & Anti-HER-2 $\left(\mathbf{N}_{3}\right)$ & HER-2 & $\begin{array}{l}\text { Dendrimers } \\
\text { (Alkyne) }\end{array}$ & & Higher cellular uptake & 138 \\
\hline & CuAAC, incorporation of anti-HER-2 & Anti-HER-2 (Alkyne) & HER-2 & Polymeric $\left(\mathbf{N}_{3}\right)$ & DOX & $\begin{array}{l}\text { 1.5-fold higher cellular uptake on SK-BR-3 } \\
\text { and } 1.3 \text {-fold higher on MCF-7 } \\
20 \% \text { higher cytotoxicity on SK-BR-3 and } \\
\text { MCF-7 }\end{array}$ & 139 \\
\hline
\end{tabular}


Table 4: to be continued

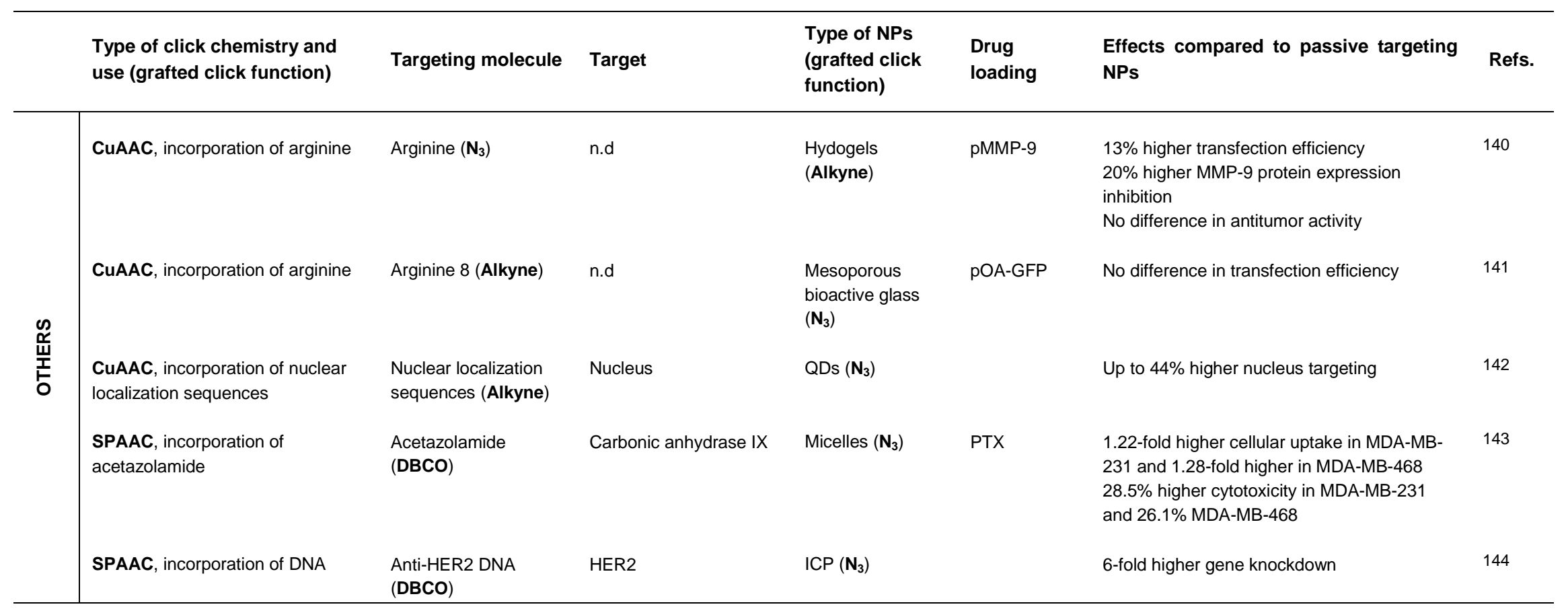

n.d: not determined, $\mathrm{N}_{3}$ : azide, DBCO: dibenzocyclooctyne, RGD: Arginine-Glycine-Aspartic acid, iRGD: Cystein-Arginine-Glycine-Aspartic acid-Lysine-Glycine-Proline-Aspartic acid-Cystein, RGDS: H-ArginineGlycine-Aspartic acid-Serine-OH, LHRH: luteinizing hormone releasing hormone, siRNA: Small interfering RNA, DNA: deoxyribonucleic acid, PSMA: prostate-specific membrane antigen, HER2: human epidermal growth factor receptor 2, GLA: glutamate urea, DOX: doxorubicin, MTX: methotrexate, PTX: paclitaxel, pOA-GFP; green fluorescent protein, MMP: matrix metalloproteinase, PSi: porous silicon, MSNs: mesoporous silica nanoparticles, AuNPs: gold nanopaticles, NMOFs: metal-organic framework nanoparticles, ICP: infinite- coordination- polymer, QDs: quantum dots. 


\subsection{Vitamins}

Vitamins are vital nutrients internalized by the metabolic activity of cancer cells due to the presence of specific overexpressed receptors on the cell surface. ${ }^{145}$ Vitamin-functionalized NPs constitute an attractive strategy for targeting tumor cells thanks to the specific recognition of vitamins by cell surface receptors, and have been employed in several targeted drug delivery approaches.

\subsubsection{Folic acid}

Folic acid (FA), also called folate or vitamin B9, is a non-immunogenic water-soluble vitamin involved in the synthesis of purines and pyrimidines of DNA as well as in cellular growth. ${ }^{146}$ This molecule, once attached to its folate receptor (FR), is internalized by endocytosis. As FRs are overexpressed in a variety of tumors (e.g. ovarian, colorectal, breast, brain, lung, etc.) and only slightly expressed in normal cells, they are one of the ligands most widely used for the active targeting of nanomaterials. Click chemistry has been used to conjugate many ligands to nanomaterials, and this approach has also been adapted to attach other frequently used cancer cell biomarkers, such as FA.

\section{Examples of CuAAC}

Several FA-based drug nanocarriers conjugated by CuAAC click reactions have been found to improve cellular uptake and therefore therapeutic efficacy in a number of studies. For instance, the folate-decorated nanoceria (FNC) co-encapsulated DOX and Ganetespib (GT) (FNC-Doxo-GT), inducing a higher rate of A549 cell death within 48 hours of incubation due to the synergistic effect compared with FNC-Doxo and FNC-GT $(90 \%, 70 \%$ and $75 \%$ cytotoxicity, respectively) (Figure). ${ }^{118}$ Moreover, fluorescence microscopy images demonstrated specific cellular uptake of FNC-Doxo-GT compared with the non-targeted PNC-Doxo-GT, for which no fluorescence was detected.

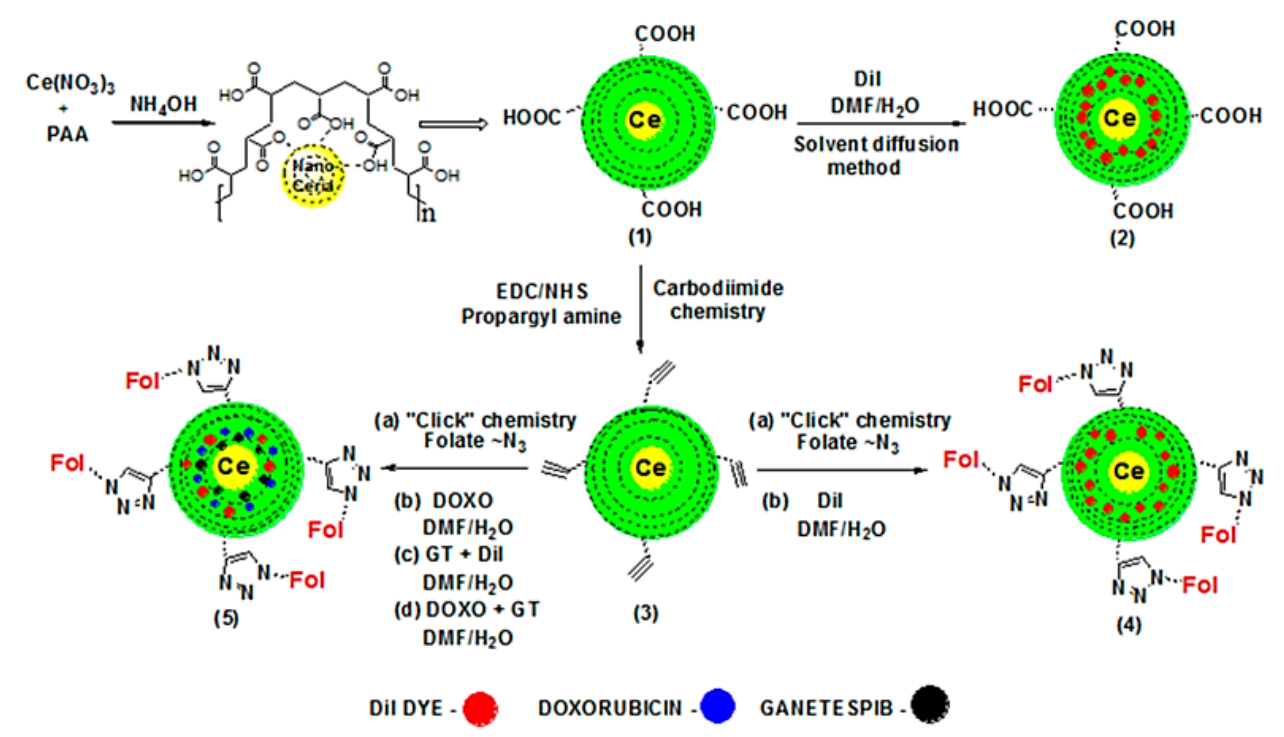

a"Click" chemistry was used to synthesize FNC, whereas a combination of drugs encapsulated using a solvent diffusion method.

Figure 12. "Click chemistry as used to synthesize FNC, whereas a combination of drugs encapsulated using a solvent diffusion method. Reprinted with permission from ref ${ }^{118}$. Copyright 2017 . American Chemical Society.

Likewise, folate conjugated NPs decorated with MTX on poly( $\varepsilon$-caprolactone)-comethoxypoly(ethyleneglycol) (P(MTXCLCL)-mPEG) demonstrated better cellular uptake than 
the non-targeted P(MTXCLCL)-mPEG (51.8\% vs 34.7\% uptake after 120 min of incubation) and a greater cytotoxic effect on MCF-7 cells $\left(\mathrm{IC}_{50}: 0.053 \mu \mathrm{g} / \mathrm{mL}\right.$ vs $\left.0.167 \mu \mathrm{g} / \mathrm{mL}\right) .{ }^{119}$

In another article, $\mathrm{Wu}$ and co-workers synthesized FA-modified core-shell nanomicelles encapsulating DOX and P-glycoprotein siRNA simultaneously. ${ }^{120}$ Fluorescent images of the micelles confirmed the FR-mediated pathway in MCF-7 cells and a significant decrease in cellular uptake when the cells were treated with FA (95.4\% vs $65.1 \%$ of DOX positive cells). Moreover, the synergistic cytotoxic effects of DOX and siRNA obtained in vitro were greater than with nanomicelles alone or free DOX (85.3\%, $41.3 \%$ and $15.7 \%$, respectively). Furthermore, these nanomicelles induced a significant improvement in tumor growth inhibition in vivo compared with free DOX $(p<0.05)$. siRNA delivery results in downregulation of P-gp protein and therefore inhibits DOX efflux from the cells.

Other FA-conjugated hollow ZnO NPs delivering PTX (FCPZnO) exhibited a higher level of cytotoxic activity than free PTX on MDA-MB-231 cells $\left(\mathrm{IC}_{50}: 11.84 \mathrm{nM}\right.$ vs $\left.7.21 \mathrm{nM}\right)$ and MCF7 cells $\left(\mathrm{IC}_{50}: 14.02 \mathrm{nM}\right.$ vs $\left.8.06 \mathrm{nM}\right) .{ }^{147}$ In murine MDA-MB-231 tumor xenografts, FCPZnO also demonstrated 4-fold higher tumor growth regression compared with PTX alone after 35 days.

Another study reports FA-modified multiblock polyurethane micelles loading DOX (SSPHPUFA-DOX) demonstrating a 2.2-fold improved cellular uptake on HeLa cells compared with SSPHPU-DOX as determined by both confocal microscopy images and flow cytometry. ${ }^{148}$ In vivo, DOX/SPION@SSPHPU-FA decreased tumor weight by up to $98.6 \%$ thanks to magnetically targeted drug delivery.

Zhang et al. synthesized FA and Cy7-modified chitosan NPs (CF7Ns) capable of both PDT and NIRF imaging. ${ }^{149}$ In vitro, HeLa cells showed higher fluorescence intensity with CF7Ns than with non-targeted C7Ns on fluorescence microscopy images and in flow cytometry. The cytotoxic effect in vitro was also significantly enhanced with NIR laser irradiation cells compared with C7Ns (75\% vs $65 \%$ for $24 \mathrm{~h}$ at $2.8 \mu \mathrm{g} / \mathrm{mL}$ ) and improved apoptosis cells for 48 hours $(70.4 \%$ vs $27.8 \%)$.

In 2016, FA-MSNs delivering DOX (NP2-BZ-FA-DOX) upon photoactivation (by UV or blue irradiation) exhibited superior cytotoxic activity against MCF-7 cells compared with nontargeted NP2-BZ-DOX (37\% vs 30\%). ${ }^{38}$ These results suggest that the targeting abilities of FA directly influence drug efficiency because of superior internalization and therefore photoactivation in the cells.

\section{Examples of SPAAC}

Similarly, BCN-FA anchored on hollow AuNPs (HAuNP-DNBA-FA) improved SERS (SurfaceEnhanced Raman Scattering) imaging on FR-positive cancer cells (i.e. KB, HeLa and A549

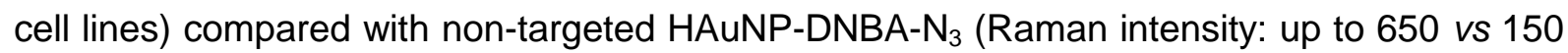
on KB cells). ${ }^{122}$

Another study using BCN-FA for SPAAC with $\mathrm{N}_{3}$-polymeric NPs (NPs-PEG-FA) also revealed a higher rate of binding on HeLa cells compared with untargeted NPs-PEG in a fluorescence microscopy study (mean fluorescence intensity (MFI): 25 vs 10). ${ }^{123}$ 


\section{Example of IEDDA}

Other MSNs anchoring FA (MSN-pHSA-CA-FA) by norbornene/tetrazine click chemistry have produced in vitro results for specific cell recognition and carbonic anhydrase-based $\mathrm{pH}$ responsive drug delivery. ${ }^{37}$ The controlled drug release is ensured by the detachment in acidic conditions $(\mathrm{pH}=5.5)$ of carbonic anhydrase during endosomal internalization. Fluorescence microscopy images of KB cells treated with MSN-pHSA-CA-FA showed a higher cellular uptake than with MSN-CA.

\subsubsection{Biotin}

Biotin is one of the vitamins required for tumor cellular growth. This molecule internalizes cells by binding to the sodium dependent multivitamin transporter (SMVT) on the cell surface, and can therefore be used for targeted drug delivery. The biotin receptor is overexpressed on the tumor cells due to the high demand of biotin for rapid tumor growth. A few examples of conjugation onto NPs for tumor-targeting exist in the literature.

\section{Examples of CuAAC}

T2-weighted magnetic resonance images of A549 cell phantoms incubated with PyreneBiotin- $\mathrm{Fe}_{3} \mathrm{O}_{4} \mathrm{NPs}$ revealed specific cell internalization. In this case, click reactions were used to develop biofunctional $\mathrm{Fe}_{3} \mathrm{O}_{4} \mathrm{NPs}$. Pyrene and biotin were grafted onto the NP surface through Diels-Alder and azide-alkyne cycoladdition respectively, with strict control over reactivity.

Moreover, a proof-of-concept for pyrene release by external magnetic field showed potential as a controlled drug delivery platform. ${ }^{150}$

In 2017, biotinylated-PEG-PLGA NPs containing DOX (BPNP) improved 4T1 cellular uptake in flow cytometry compared with non-biotinylated DPNP (MFI of 214 vs 113) and were more cytotoxic after 24 hours (IC50: $91.5 \mathrm{nM}$ vs $131.8 \mathrm{nM}$ ). ${ }^{121}$ In vivo, BPNP demonstrated higher tumor uptake on mice bearing 4T1 breast tumor cells than DPNP (maximum tumor concentration: $49.4 \mathrm{ng} / \mathrm{mg}$ vs $22.45 \mathrm{ng} / \mathrm{mg}$ ); the level of antitumoral activity was higher, leading to increased tumor volume regression ( 99.36 to $29.74 \mathrm{~mm}^{3}$ ) compared with DPNP (102.72 to $68.43 \mathrm{~mm}^{3}$ ) and free DOX $\left(100.43\right.$ to $\left.76.38 \mathrm{~mm}^{3}\right)$.

\section{Examples of SPAAC}

Other articles mention biotin-clicked NPs by SPAAC for biosensing applications to amplify RNA and DNA detection with AuNPs ${ }^{151}$ or UCNPs. ${ }^{152}$

\subsection{Carbohydrates or polysaccharides}

One possibility for the preparation of drug delivery targeting NPs is to use carbohydrates specifically directed against cell surface receptors (i.e. lectins). There are different classes of targeting molecules for binding lectins including monosaccharides (e.g., galactose, mannose, fucose, sialic acid), disaccharides (e.g., lactose and N-acetyllactosamine), and polysaccharides (e.g., hyaluronic acid, pullulan, dextran, chitosan). Lectins are known to be involved in the growth and metastasis of tumors and constitute an interesting targeting approach using the endocytosis process. ${ }^{153,154}$ Several carbohydrate NPs have thus been 
synthesized using click chemistry for specific targeting therapies and, for example, have recently been reported to have produced glyco-gold NPs. ${ }^{155}$ Functionalization using CuAAC click chemistry is only reported for the studied period.

\subsubsection{Hyaluronic acid}

NPs containing hyaluronic acid (HA) are attractive candidates for therapeutic applications. $\mathrm{HA}$ is a natural anionic polysaccharide, an extracellular constituent of connective tissues and is able to bind the CD44 receptor overexpressed in several tumor cells. Furthermore, HA is often used as a carrier for intracellular controlled drug release through degradation by hyaluronidase (HAase). ${ }^{156}$

\section{Examples of CUAAC}

Sun et al. investigated this strategy for engineering tumor-targeted siRNA delivery nanosystems (Figure13). ${ }^{124}$ CuAAC enabled easy conjugation of alkyne-HA polymers (alk$\mathrm{HA}$ ) onto lipid-based complexes encapsulating siRNA (RSC) using the $\mathrm{N}_{3}$-modified cholesterol present on the surface (RSC-HA).

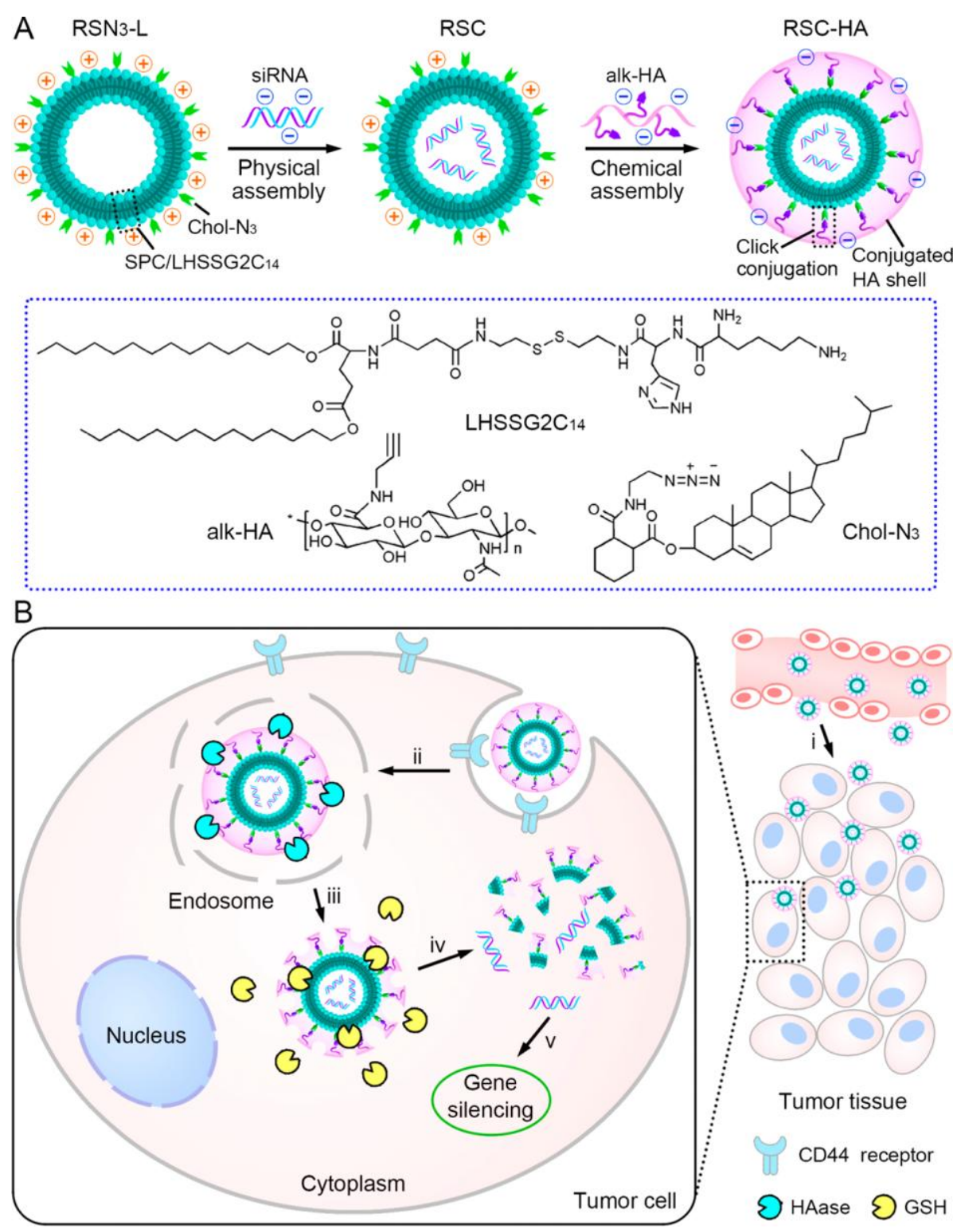


Figure 13. (A) Schematic illustration of the "collaborative assembly" strategy for construction of an siRNA delivery system consisting of a lipidpolymer hybrid nanocarrier via a combination of an electrostatically driven physical assembly and a click reaction-mediated chemical assembly. (B) Schematic illustration of tumor-targeted siRNA delivery by RSC-HA. (i) Accumulation of RSC-HA at the tumor site; (ii) endocytosis of RSC-HA into the tumor cells; (iii) HAase-mediated degradation of the HA shell of RSC-HA and endosomal escape into the cytoplasm; (iv) GSH-triggered disassembly of RSC-HA and release of the complexed siRNA in the cytoplasm; and (v) gene silencing induced by the released siRNA. Reprinted with permission from ref ${ }^{122}$. Copyright 2017 American Chemical Society

Flow cytometry revealed a significant improvement in the cellular uptake of RSC-HA compared with RSC in vitro on A549 cells (MFI 140 vs 80). The efficiency of siRNA genesilencing also improved significantly with $55 \%$ downregulation of mRNA expression for RSCHA compared with $40 \%$ for RSC, and a higher rate of anticancer activity was observed in vitro (total apoptotic ratio: $23.48 \%$ vs $13.36 \%$ ). Moreover, vectors with HA polymers grafted onto the liposome by click chemistry showed greater in vivo stability in the blood, resulting in efficient tumor targeting, compared with RSC/HA in which HA was physically adsorbed on the complex. 2-fold higher tumor uptake and tumor growth inhibition were obtained after 10 days of treatment compared with non-targeted RSC, with approximately $30 \%$ superior genesilencing efficiency.

\subsubsection{Glycosides}

Sugars can enhance binding affinity with lectin receptors thanks to a phenomenon called the "cluster glycoside effect", which causes diverse copies of glycoside clusters to interact together to enhance recognition. ${ }^{157}$ Dendritic multivalent glycosides are widely used in a number of biomedical approaches. ${ }^{158}$ Only examples of CuAAC click chemistry during the studied period are therefore described herein.

\section{Examples of CuAAC}

Rajakumar et al. incorporated glucose into dendrimers by CuAAC and showed cardioprotective and anti-diabetic properties. ${ }^{159}$

Interestingly, Kong et al. synthesized mannose and galactose-modified $\mathrm{SiO}_{2} \mathrm{NPs}$, and demonstrated the concept of a quantitative fluorine NMR method to determine the density of carbohydrates incorporated by CuAAC. ${ }^{160}$ CuAAC was also used to incorporate thiosialoside on fullerenes directed against the influenza virus neuraminidase, despite a moderate antiviral activity. ${ }^{161}$

\subsection{Aptamer ligands}

Nucleic acid aptamers are single-stranded oligonucleotides (DNA or RNA) widely used as therapeutic molecules. They can target a large variety of specific proteins overexpressed by cancer cells leading to possible internalization. Aptamers can be chemically synthesized and easily modified with a broad range of chemical functions. ${ }^{162}$ Aptamer-decorated nanocarriers thus show promise as drug delivery systems. Nevertheless, most of the strategies employed for surface functionalization are based on electrostatic interactions and suffer from possible instability. In this context, click chemistry was able to ensure DNA/RNA surface functionalization of nanomaterials. For instance, CuAAC demonstrated effective bioconjugation of DNA on polymeric QDs for hybridization. ${ }^{163}$

\section{Examples of SPAAC}


Among the aptamers currently employed, AS14111 is specific to the nucleolin receptor. ${ }^{125}$ In one study, AS14111 was conjugated by SPAAC on metal-organic framework NPs (NMOFs) for specific DOX release in acidic conditions ( $\mathrm{pH}$ 5.0). ${ }^{164}$ Targeted NMOFs demonstrated higher cytotoxicity than non-targeted NMOFs in vitro on MDA-MB-231 cancer cells after three days (45\% vs $35 \%)$.

In another study, SPAAC was also investigated to conjugate DNA on NMOFs (MOF-DNA) and achieved a 5-fold increase in the cellular uptake of $14 \mathrm{~nm}$ MOF-DNA, compared with MOF alone. ${ }^{126}$

In 2016, UCNPs-MB/Dox was developed with the conjugation of a TK1 mRNA-specific molecular beacon (MB) bearing a quencher (BHQ-1) and an alkene handle modified UCNP through click reaction. These original UCNPs showed $57 \%$ specific cytotoxicity on MCF-7 cancer cells compared with no cytotoxicity for LO2 normal cells. ${ }^{165}$

Due to the high affinity and specificity of aptamers, they have been widely investigated for protein detection assays. For instance, Chen et al. designed double-stranded DNA-MSNs embedding FITC (dsDNA-FITC-MSN) to elaborate thrombin biosensors. FITC was released from the pores only after specific dsDNA-thrombin recognition, suggesting potential use as a stimulus responsive system to detect thrombin in serum samples. ${ }^{36}$

Akiel et al. used SPAAC to functionalize $\mathrm{N}_{3}$-NDs with DBCO-single strand DNA for subsequent hybridization to complementary strands. ${ }^{166}$ Specific DNA association can be characterized by a different X-band EPR spectroscopy profile compared with the nonhybridized nanomaterial, making them promising sensor platforms.

\section{Examples of thiol-ene}

Other authors have anchored aptamers on NPs through thiol-ene click reactions in order to detect and capture bovine serum-albumin (BSA). ${ }^{29} \mathrm{NPs}$ were combined with a molecularly imprinted polymer layer able to entrap molecules such as proteins in a complex matrix. Thiolene click chemistry was also successfully applied to colloidal NPs for aptamer bioconjugation. ${ }^{46}$

\subsection{Peptides}

\subsubsection{RGD peptides}

Among the various types of peptides, integrins are a large class of cell adhesion receptors comprising 24 different peptides. They are very useful for targeting tumor sites due to their role in disease progression. ${ }^{167}$ The tripeptide sequence Arg-Gly-Asp (RGD) is often used as a ligand owing to its affinity with a wide variety of integrins that promote cellular adhesion and internalization. ${ }^{168}$

\section{Examples of CuAAC}

In 2018, Jia and co-workers improved the ability of exosomes embedding RGE-modified SPION (Super Paramagnetic Ion Oxide Nanoparticle) and Curcumin (RGE-Exo-SPION/Cur) to target glioma cancer cells. ${ }^{128}$ They demonstrated a much higher targeting ability with RGEExo in vitro on U251 cells compared with free-Exo (close to $100 \%$ vs 40\%) and 3-fold higher 
tumor targeting in vivo on glioma tumor-bearing mice 4 hours after intravenous injection. Cytotoxicity was found to be significantly higher with RGE-Exo than with free-Exo $(60 \%$ cell inhibition vs $40 \%)$, as was tumor volume suppression $\left(70 \mathrm{~mm}^{3}\right.$ to $3 \mathrm{~mm}^{3}$ vs $70 \mathrm{~mm}^{3}$ to $\left.20 \mathrm{~mm}^{3}\right)$ with no tumor regression for 28 days.

CuAAC conjugation of RGD peptides is usually performed using polymers and PEG-coated NPs with alkyne peripheral functional groups. In a recent study, the potential of RGD targeting was reported on micelles loaded with PTX comprising polymers and $\mathrm{N}_{3}$-PEG. ${ }^{129}$ RGD achieved 3-fold higher 4T1 cellular uptake compared with non-targeted NPs thanks to MMP-2 proteolytic cleavage, and superior cytotoxicity ( $\mathrm{IC}_{50}: 79 \mathrm{ng} / \mathrm{mL}$ vs $\left.1130 \mathrm{ng} / \mathrm{mL}\right)$. In vivo, RGD-NPs exhibited higher tumor growth inhibition with a 1.9-fold volume increase after 24 days compared with the 16-fold increase for non-targeted NPs.

In 2016, Arriortua et al. investigated the conjugation of $\mathrm{N}_{3}-\mathrm{RGD}$ with amino-alkyne polymeric iron NPs $\left(\mathrm{Fe}_{3} \mathrm{O}_{4} @ P M A O \_R G D\right) .{ }^{130}$ The hyperthermia properties of $\mathrm{Fe}_{3} \mathrm{O}_{4} @ P M A O \_R G D$ NPs significantly increased the necrosis of liver and colorectal tumors (up to 16\%), compared with saline groups, under a magnetic field without damaging the hepatic tissues.

Fluorescent probes have been successfully used in the field of targeting NPs and afford new opportunities in biomedicine such as specific cancer diagnosis. In this context, nanodiamonds (NDs) constitute interesting fluorescent systems for bioimaging applications because of their optical crystal properties responsible for NIRF. Slegerova et al. described fluorescent NDs coated with an alkyne-copolymer allowing incorporation of both $\mathrm{N}_{3}$-cyclic RGD peptides and fluorescent molecules (Alexa Fluor 488) (FNDs-cRGD) for glioma cancer cell imaging. ${ }^{131}$ These FNDs-cRGD demonstrated 12-fold higher U-87 MG cellular binding compared with FNDs. A similar strategy to introduce $\mathrm{N}_{3}$-labeled ${ }^{125}$ I-RGD or FITC probes for prostate cancer cell imaging was applied by Rehor et al. using NDs coated with alkyne-PEGs and polymers. ${ }^{169}$

In 2017, multifunctional RGD- $\mathrm{Fe}_{3} \mathrm{O}_{4}$ NPs also showed 1.4-fold higher cellular binding compared with non-targeted NPs on BT-20 cells. ${ }^{132}$

Another study demonstrated enhanced cellular uptake of porous silicon (PSi) NPs loaded with three different molecular weight targeting peptides (i.e. $\mathrm{N}_{3}$-functionalized iRGD, RGDS and poly(glutamic acid)) on endothelial EA.hy926 and U87 MG cells. ${ }^{127}$

Oz et al. synthesized NPs conjugated with cRGD and BODIPY fluorescent dye by CuAAC and thiol-maleimide click chemistry for the imaging of MDA-MB-231 breast adenocarcinoma cells. ${ }^{170}$ They demonstrated higher cellular uptake in fluorescence microscopy with the targeting $\mathrm{CRGD}$ peptide.

\section{Examples of SPAAC}

Wang et al. compared active and passive cellular targeting to improve retention and augment the efficacy of the therapy at the target site. They reported a new theranostic and multifunctional porous silicon (PSi) NP suitable for both imaging and delivery of a therapeutic agent (i.e. sorafenib). ${ }^{134}$ The surface was modified using SPAAC to introduce the DOTA chelators necessary for ${ }^{111} \mathrm{In}$-radiolabeling for SPECT/CT imaging. In a second step, the 
targeting peptide iRGD was inserted to form PSi-iRGD-NPs (Figure). The PSi-iRGD-NPs exhibited superior tumor-specific accumulation in prostate cancer cells (PC3-MM2) compared with PSi-NPs 27 hours after intravenous injection (4.4 vs 2.7 tumor-to-muscle ratios of the radioactivity). However, the NPs demonstrated similar levels of tumor growth inhibition after intravenous and intratumoral injection.

(a)

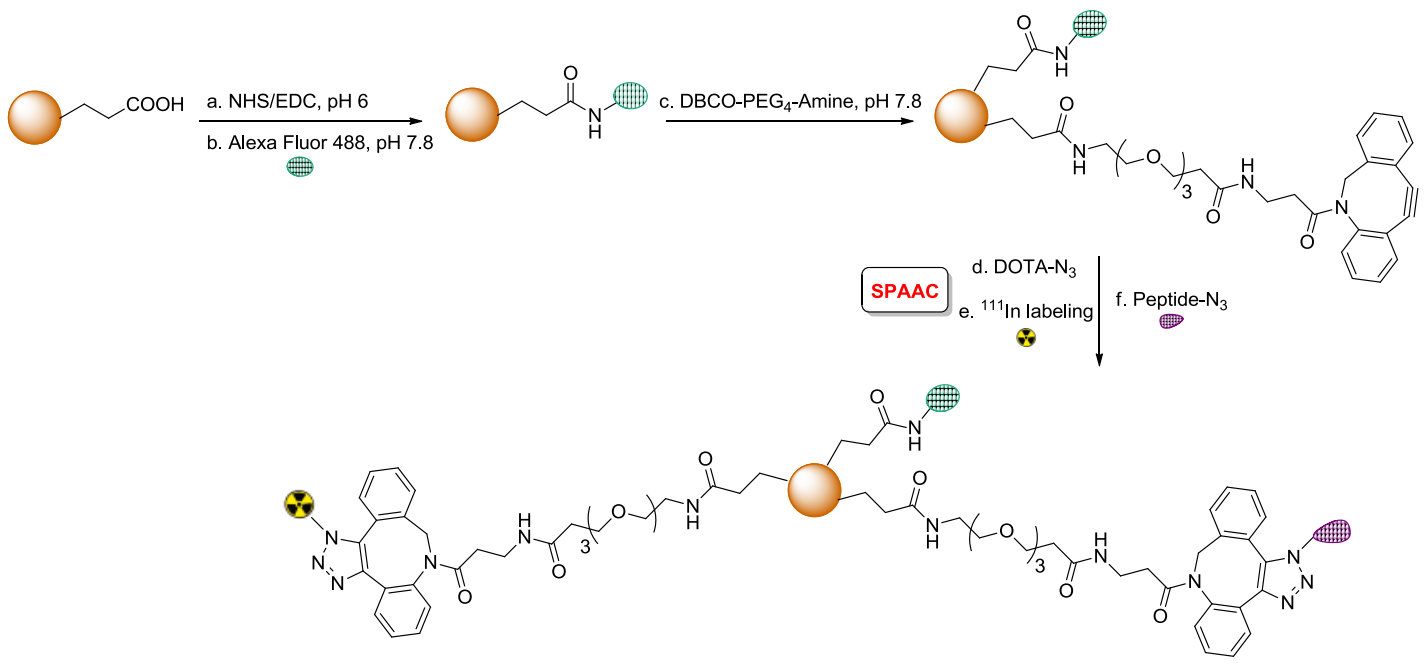

(b)
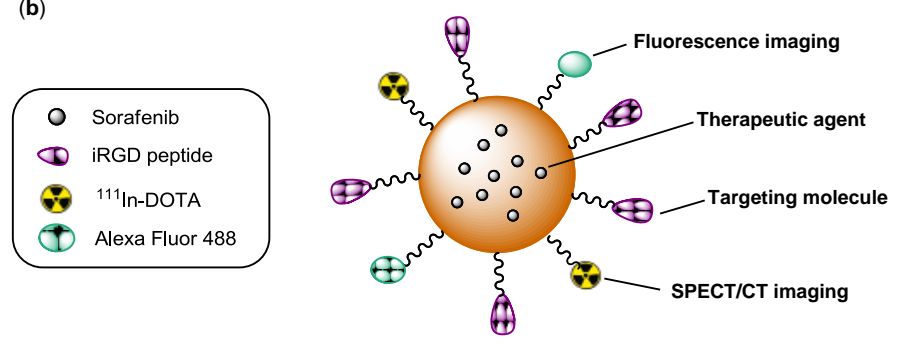

Figure 14. (a) Synthesis and (b) schematic structure of the multifunctional nanocarrier encapsulating Sorafenib, functionalized with Alexa Fluor 488 and labeled ${ }^{111}$ In-DOTA and iRGD peptide via SPAAC click chemistry. Figure modified from ref ${ }^{134}$.

An efficient drug delivery system was also created by using BCN-functionalized PSi-NPs to conjugate $\mathrm{N}_{3}$-RGD derivatives (TCPSi-RGDS/iRGD). ${ }^{135}$ This approach led to a significant improvement (20\% higher) in cellular uptake for TCPSi-RGDS and $15 \%$ higher cell growth inhibition with sorafenib on EA.hy926 endothelial cells compared with non-targeted NPs.

\subsubsection{Cell-penetrating peptide (CPP)}

Cell penetrating peptide (CPP) was recently identified as a promising ligand for targeting cancer therapy. Natural and synthetic peptides are widely used to enhance cellular uptake via the endocytosis process and are conjugated to deliver cargo-based nanomedicines in particular. ${ }^{171}$ Trans-activating transcriptional activator (Tat) is one of the CPPs used for targeting delivery systems. CPP is a positively-charged peptide derived from the Human Immunodeficiency Virus 1 (HIV-1) and can access the cell nuclei to exercise its translocation activity. ${ }^{172}$

\section{Examples of CuAAC}

In 2015, Liu et al. described PSi nanocomposites constituted of an acetylated dextran matrix (AcDX), degradable in acidic conditions, embedding the three therapeutic molecules (i.e. 
Sorafenib, MTX and PTX) currently used to treat breast cancer. ${ }^{173}$ They used oxime click chemistry to include cell-penetrating peptides (CPP) as receptor internalization pathways to finally obtain PSi@AcDX-CPP NPs.

Experiments carried out at two different levels of $\mathrm{pH}$ (i.e. $\mathrm{pH} 7.4$ and $\mathrm{pH} 5.0$ ) indicated controlled release of the cargos only at $\mathrm{pH} 5.0$ because of hydrolysis of the polymeric matrix.

The advantage of adding CPP-targeting moieties to improve internalization was confirmed by cell uptake studies on two breast cancer cell lines comparing PSi@AcDX-CPP and PSi@AcDX NPs (MCF-7: MFI of 2000 vs 250; MDA-MB-231: MFI of 1550 vs 250). PSi@AcDX-CPP significantly improved proliferation inhibition compared with the nontargeting PSi@AcDX (cell viability for MCF-7 and MDA-MB-231: 60\% vs no cytotoxic effect).

In the same year, $\mathrm{N}_{3}$-Tat was used to functionalize micelles loaded with DOX via CuAAC. ${ }^{174}$ Specific release of the drug was achieved by two types of amide hydrolysis. The first was carried out by the nanocarrier's positively-charged amphiphilic diblock copolymer poly $(\mathrm{L}-$ lysine)-block-poly(L-leucine) (PLL-PLLeu). This polymer became negatively charged at $\mathrm{pH}$ $\sim 6.5$ in the extracellular microenvironment of the tumor, allowing internalization by the tumor cells. The second was carried out by the Tat peptide amidated by succinyl chloride (Tat(SA)). After uptake by the cells, the peptide was activated at pH 5.0 in the endosome through SA hydrolysis. This $\mathrm{pH}$-sensitive amide allowed subsequent activation of nuclear targeting and DOX release. Confocal images of the HeLa cells showed more red fluorescence of DOX with targeting NPs than with non-targeting NPs and demonstrated higher nuclear delivery of DOX for 24 hours (400 ng vs $140 \mathrm{ng}$ of DOX $/ 10^{6}$ cells respectively).

One robust nanomedicine application, the delivery of small interfering RNA (siRNA), is a promising tool for silencing genes overexpressed in cancer. In vitro studies on the neurotropic rabies virus confirmed the higher level of delivery of CPP anandamide and siRNA-functionalized dendrimers to neural stem cells. ${ }^{175}$ They efficiently transfected siRNA (up to 2-fold higher relative mRNA concentration) leading to a 2-fold higher decrease in virality compared with the non-targeting anandamide system.

\subsubsection{Other peptides}

Other bioactive peptide ligands are used for a variety of pathologies and applications. Wan's group demonstrated highly efficient and highly chemoselective peptide conjugation with dendrimers using SPAAC, associated with no alteration of in vitro and in vivo bioactivity. ${ }^{176}$

Alkyne-functionalized polylysine dendrons were prepared for click chemistry with $\mathrm{N}_{3}$-PEGchain-modified $X$-MrlA analogues, disulfide-rich $X$-conotoxin peptides implicated in the treatment of neurological disorders, by selectively inhibiting the human norepinephrine transporter. ${ }^{177}$ Other alkyne-functionalized lysine dendrons using a solid-phase $\mathrm{Cu}(\mathrm{I})$ catalyzed for azide peptide conjugation have shown great potential for future immunoassays. ${ }^{178}$ The targeting potential of peptide for vectorized drug delivery into prostate cancer cells using PSMA-targeted PAMAM dendrimers has also been reported by other investigators. ${ }^{136}$ These nanocarriers were obtained by SPAAC conjugation of $\mathrm{N}_{3}$-glutamate urea (GLA) targeting peptides and $\mathrm{N}_{3}-\mathrm{MTX}$. They demonstrated approximately 8-fold higher cellular uptake compared with non-targeted NPs. In another study, synthesis of luteinizing hormone releasing hormone (LHRH) peptide-functionalized NPs encapsulating recombinant 
human tumor suppressor proteins (p53) showed enhanced anticancer effects in vitro on MDA-MB-231 cells compared with the non-targeted version (almost $100 \%$ vs up to $30 \%$ cell growth inhibition). ${ }^{137}$

Another article also showed enhanced cellular uptake with polymeric DOX-NPs conjugated with a nucleolin-targeting F3-peptide overexpressed in tumor cells (9L, MCF-7 and NCl/ADRRES cells). ${ }^{133}$ A review published by Tang and Beker ${ }^{179}$ contains more examples of click reactions for peptide-functionalized nanomaterials prior to 2014.

\subsection{Protein ligands}

Glycoprotein transferrin is one type of protein used as a targeting ligand because human transferrin receptors are abundantly expressed in tumor cells. ${ }^{180}$

\section{Examples of SPAAC}

After bioconjugation with quantum dots ${ }^{181}$ and magnetic nanogels ${ }^{182}$ using SPAAC, protein ligands reduce side effects and improve the delivery of therapeutic drugs thanks to the receptor-mediated endocytosis pathway. Epifluorescence images of HeLa cells show that bioconjugation of $\mathrm{N}_{3}$-QDs with DBCO-transferrin promotes cellular uptake. In the second study, SPAAC between $\mathrm{N}_{3}$-PEG-transferrin and bicyclononyne-NPs (BCN-NPs) resulted in up to $90 \%$ conjugation efficiency. Magnetic NPs were thus used to detect and capture circulating tumor cells overexpressing transferrin receptors.

\section{Example of IEEDA}

Tz-norbornene click chemistry has been successfully applied to hydrogel NPs to anchor proteins such as fluorescent ovalbumin (Tz-Oval), alkaline phosphatase (Tz-ALP) and glucose oxidase (Tz-GOx) for potential applications in tissue engineering and glucose biosensing. ${ }^{183}$ These protein-functionalized NPs showed higher bioactivity than their nonfunctionalized analogs NF-Oval, NF-ALP and NF-GOx (5-fold, 12-fold and 9-fold increase in bioactivity, respectively) at higher concentrations.

\subsection{Monoclonal antibodies (mAbs)}

Monoclonal antibodies are immunoglobulin (lg) proteins whose $\lg G$ form remains a key element of many targeted therapies. The growing interest in using mAb-conjugated NPs for active targeting is mainly due to their ability to improve tumor accumulation. Full mAbs, as well as mAbs fragments, such as $F^{\prime}(a b)_{2}, F^{\prime}(a b)$ or diabody structures, can be used to target tumors. Many strategies are employed to attach these mAbs or mAbs fragments to NPs; they must ensure optimal orientation for subsequent functional binding and antigen (Ag) recognition. The thiol-maleimide reaction is easily employed with mAb because of the thiol groups that naturally exist in cysteine residues. ${ }^{184}$ However, the main restriction to the use of this reaction is the potential hydrolysis of the maleimide into an unreactive maleic acid amide that may reduce conjugation efficiency. In this context, click chemistry has been widely used for covalent linkage by prior mAb derivatization with click functions. ${ }^{185-187}$ This conjugation strategy offers the possibility of generating stable, efficient interactions between the two entities with minimal purification steps and high yields. ${ }^{30,188}$ Among the mAbs conjugated by 
click chemistry, anti-EGFRs have been most widely studied. ${ }^{189}$ They are used to target and treat tumors thanks to their involvement in cancer progression.

\section{Examples of CuAAC}

In 2016, $\mathrm{N}_{3}$-anti-HER-2 mAb was covalently introduced on alkyne PAMAM dendrimers by CuAAC; AuNPs and Gd were also entrapped in the dendrimer and DOTA, respectively (AuG5-Gd-Herceptin) (Figure). ${ }^{138}$ Confocal images of A549 cells exhibited higher internalization with Au-G5-Gd-Herceptin than with Au-G5-PEG-Alkyne-DOTA-Gd-NHAc.

The same observations were made with PLGA-Phis-PEG NPs conjugated with anti-HER-2 (PNH) by CuAAC and encapsulating DOX. ${ }^{139} \mathrm{PNH}$ showed approximately 1.5-fold and 1.3fold higher cellular uptake on SK-BR-3 and on MCF-7 cell lines respectively compared with PN after 120 min of incubation. They also demonstrated higher cytotoxicity effects (SK-BR-3: $70 \%$ vs 50\%; MCF-7: $65 \%$ vs $45 \%$ cell growth inhibition) at $0.5 \mu \mathrm{g} \mathrm{DOX/well.}$

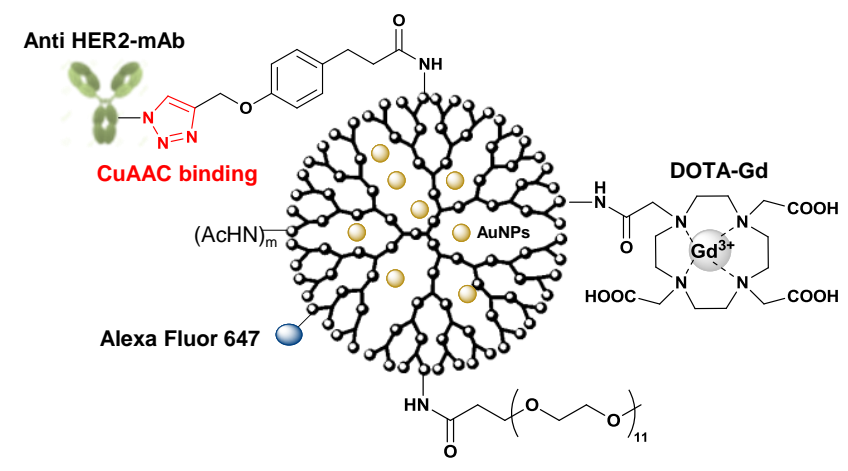

Figure 15. Schematic structure of the multifunctional NP-imaging agent Au-G5-Gd-Herceptin. Figure modified from ref ${ }^{138}$.

\section{Examples of SPAAC}

Kotagiri et al. demonstrated that the SPAAC reaction produced anti-EGFR-QDs with a higher yield than anti-EGFR-QDs obtained by traditional thiol-maleimide conjugation which depends on hydrolysis $(88 \%$ vs $60 \%) .{ }^{55}$ Moreover, the stability of DBCO and azide functions in aqueous solution resulted in a larger number of mAbs per QD (up to 8.4 vs 3.9). Confocal images of BxPC-3 and MDA-MB-231 cells showed higher fluorescence intensity for the clickconjugated mAb-QDs. This was also the case for $\mathrm{SiO}_{2} \mathrm{NPs}$, where a larger amount of antiHER-2 (Trastuzumab) was conjugated by SPAAC than by EDC/NHS coupling (50 to $70 \%$ vs 1 to $20 \%$ ) resulting in a targeting capacity 8-fold higher on MDA-MB-231 breast cancer cells. ${ }^{190}$

In 2016, Ma et al. described efficient synthesis of polymeric-coated QDs bioconjugated with anti-EGFR (QD-EGFR Ab) by SPAAC; selective attachment of the membrane region was achieved compared with the QD-IgG control. ${ }^{163}$ SPAAC was also performed with antiVEGFR-functionalized QDs for in vitro imaging of angiogenic receptors. ${ }^{191}$ Qiao and his team synthesized $\mathrm{NaGdF}_{4}: \mathrm{Yb}, \mathrm{Er} @ \mathrm{NaGdF}_{4}$ upconversion NPs (UCNPs) conjugated with the antigastric tumor antibody, $\mathrm{MGB}_{2}$, and demonstrated both specific optical and MR imaging properties in vivo on implanted SGC7901-Luc cells, thanks to their targeting capacity. ${ }^{192}$ 
mAb-conjugated NPs have also been investigated for biosensing applications because of their high affinity and specificity towards biomarkers of interest. Click chemistry has proved to be a promising strategy for immobilizing such probes for immunoassays. ${ }^{30,193}$

\subsection{Other nano-conjugates}

\section{Examples of CUAAC}

In another study, the authors synthesized dendrons and a-cyclodextrin-based hydrogels, including arginine conjugated by CuAAC, for pMMP-9 plasmid delivery (MPEG-PLLDArg/pMMP-9 and MPEG-PLLD-Arg/pMMP-9/a-CD). ${ }^{140}$ They demonstrated significantly higher transfection of HNE-1 cells with MPEG-PLLD-Arg/pMMP-9 than with PEI-25k/pMMP-9 (up to $62 \%$ vs $49 \%$ ) and consequently higher inhibition of MMP-9 protein expression $(70 \%$ vs $50 \%)$.

In vitro transfection assays with MPEG-PLLD-Arg/pMMP-9/a-CD transfected up to $72 \%$ of cells at 12 hours and induced superior HNE-1 cell apoptosis compared with PEI-25k/pMMP-9 (46\% vs $31 \%$ ). However, tumor growth inhibition in vivo was similar with both formulations. In 2017, Li et al. also conjugated octaarginine 8 on mesoporous bioactive glass (MBG-PGArg8) by CuAAC for gene transfection but found no significant difference in transfection efficiency with MBG-PG-Arg8 compared with MBG-NH 2 (32\% vs 29\%). ${ }^{141}$

Targeting efficiency and intracellular internalization have also been achieved by Maity and Stepensky. ${ }^{142}$ They designed efficient QDs decorated with nuclear localization sequences (QD-NLS) by CuAAC, conferring specific nucleus accumulation. The intracellular efficiency of three different QD formulations was compared on HeLa cells: QD-COOH, QD-azide and QDNLS. QD-NLS improved cell targeting efficiency by up to $44.2 \%$ compared with QD-COOH and QD-N $\mathrm{N}_{3}$ (MFI of $11.4,3.60$ and 6.07, respectively).

\section{Examples of SPAAC}

In 2018, Tatiparti et al. described PTX-loaded nanomicelles (HSA-PTX-AZT) targeting carbonic anhydrase IX (CA IX) directed against triple negative breast cancer cells. ${ }^{143}$ The NPs functionalized with acetazolamide (AZT, hypoxia marker of CA IX) by SPAAC showed higher apoptosis compared with non-targeted NPs in vitro on MDA-MB-231 and MDA-MB468 (MDA-MB-231: $74.8 \%$ vs $46.3 \%$; MDA-MB-468: $75.3 \%$ vs $49.2 \%$ ) due to receptormediated cell uptake. Fluorescence spectroscopic studies also demonstrated significantly higher cellular uptake (MDA-MB-231: MFI 120 vs 98; MDA-MB-468: MFI superior to 100 vs $\sim 78$ ) at 16 hours. In 2015, anti-HER2 DNA-labeled ferric nitrate infinite-coordination-polymer NPs (HER2-ICP) were developed and found to reduce HER2 expression by up to $80 \%$ with $10 \mu \mathrm{M}$ DNA on SKOV-3 cells compared with no efficient gene knockdown with the NonTarget-ICP. ${ }^{144}$ 


\section{Bioorthogonal chemistry and pretargeting (PT) systems for NP delivery}

This section concerns the term bioorthogonal chemistry and not click chemistry. Bioorthogonal chemistry means that the formation of the covalent link between the two entities does not need a catalyst and may occur in physiological conditions. The previous sections of this paper cite several articles mentioning SPAAC and IEDDA systems, which are characteristic of bioorthogonal chemistry. In these previous examples, the click reaction was used to build the NP for both passive and active targeting. In this fourth section, bioorthogonal chemistry is described for pretargeting (PT) systems. The PT approach using bioorthogonal chemistry is another extremely promising solution to improve tumor targeting.

Due to the limited number of cellular receptors and TME heterogeneity, bioorthogonal chemistry has been attracting growing interest over the past few years. The delivery of NPs onto the surface of living cells has improved the targeting efficiency and delivery of both imaging agents and drugs. ${ }^{50,64,194}$ This two-step approach offers several advantages over the conventional, direct covalent coupling of targeting ligands. It prevents NP aggregation and cross-linking between macromolecular entities (mAbs, avidin, etc.). NPs with bioorthogonal functions are often easier to synthesize and purify, requiring only removal centrifugation unlike direct conjugates, which often require optimization processes to maximize the ligandto-NP ratio without compromising colloidal stability. Direct labeling of mAbs/avidin on NPs can decrease Ag recognition because of steric hindrance compared with mAb. Moreover, for larger NPs this can lead to massive conjugates that are less easily delivered to the TME. This strategy could also improve the number of NPs per mAb and therefore amplify the signal per biomarker. Compared with traditional antibody-NP conjugation, this method can be applied to a broader range of pathologies depending on the suitable mAb.

PT approaches applied to NPs rely on specific, covalent interactions between two reactive groups (i.e. bioorthogonal entity or tag), one usually carried by the nanomaterial reacting with another incorporated onto the molecule of interest (e.g. imaging, therapeutic agent or targeting molecule). This two-step strategy consists in injecting a tagged targeting agent (i.e. NP or molecule of interest), which will accumulate in sufficient quantities on the cells, then injecting a second tagged agent (i.e. NP or molecule of interest), which specifically and covalently binds through the bioorthogonal reaction. The different PT approaches for potential imaging, diagnosis and drug delivery applications reported from 2010 to the present are summarized in Figure and detailed in the section below. In most studies, mAbs are widely used as primary targeting agents (Figurea), but NPs are also increasingly used as platforms for imaging and therapeutic agents (Figureb). 


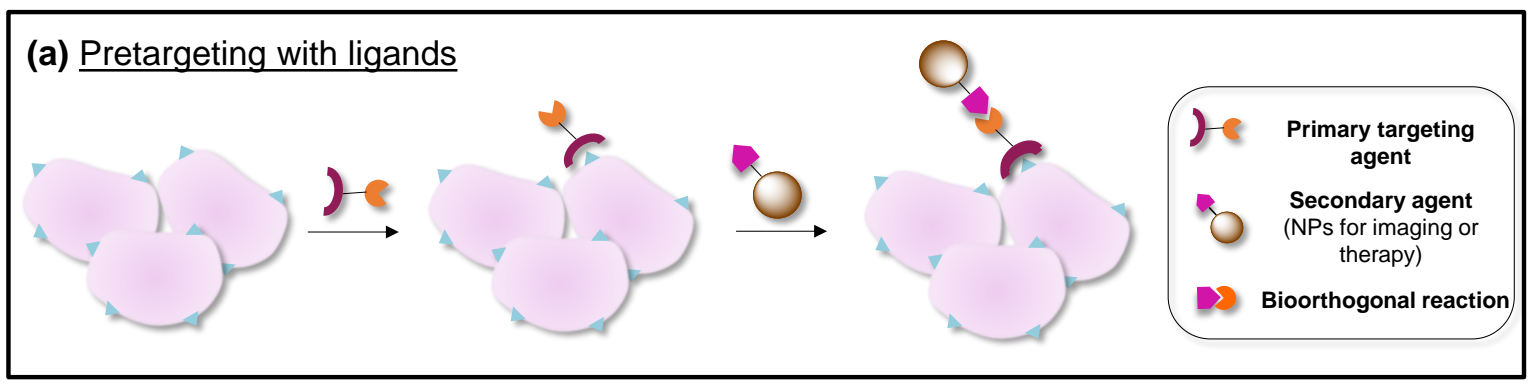

(b) Two-step targeting with NPs as

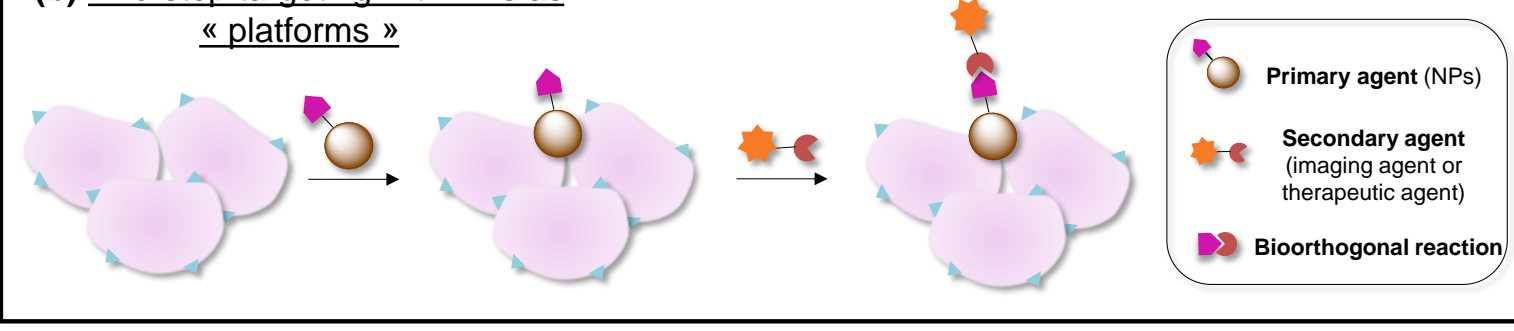

Figure 16. Different pretargeting strategies for imaging, diagnosis and therapy. (a) Firstly, targeting ligands are injected to enable specific accumulation in tumors. Secondly, NPs are administered and will covalently bind with the suitable tag on the targeting ligands. (b) Firstly, NPs are administered to accumulate in cancer cells either by passive or active targeting. Secondly, imaging or therapeutic agents are injected and will bind with the reactive, complementary tag previously deposited on the NPs.

\subsection{PT approaches with ligands as targeting agents}

An overview of recent reports using PT approaches with ligands as targeting agents is presented in more detail in this section and summarized in Table 5.

Table 5. General overview of PT approaches using ligands as pretargeting agents.

\begin{tabular}{|c|c|c|c|c|c|}
\hline & $\begin{array}{l}\text { Bioorthogonal } \\
\text { system }\end{array}$ & $\begin{array}{l}\text { Primary tumor-targeting agent } \\
\text { for the first step of the PT } \\
\text { approach }\end{array}$ & $\begin{array}{l}\text { Complementary agent for the } \\
\text { second step of the PT } \\
\text { approach }\end{array}$ & Application & Refs. \\
\hline \multirow{8}{*}{$\begin{array}{l}0 \\
\frac{⿱ 亠 ⿻}{\alpha} \\
\text { के }\end{array}$} & \multirow{6}{*}{$\mathrm{DBCO} / \mathrm{N}_{3}$} & $\mathrm{Ac}_{4} \mathrm{MAnNAz}\left(\mathbf{N}_{3}\right)$ & Liposomes (DBCO) & $\begin{array}{l}\text { In vitrolin vivo optical imaging } \\
\text { and drug delivery }\end{array}$ & 195 \\
\hline & & $\mathrm{Ac}_{4} \mathrm{MAnNAz}\left(\mathbf{N}_{3}\right)$ & Gelatin-oleic-DOX NPs (DBCO) & $\begin{array}{l}\text { Drug delivery and anticancer } \\
\text { effect }\end{array}$ & 45 \\
\hline & & $\mathrm{Ac}_{4} \mathrm{MAnNAz}\left(\mathbf{N}_{3}\right)$ & $\begin{array}{l}\text { Liposome DBCO or liposome } \\
\text { TCO (2 step-targeting) }\end{array}$ & In vivo imaging and therapy & 196 \\
\hline & & $\mathrm{Ac}_{4} \mathrm{ManDBCO}(\mathrm{DBCO})$ & QDs $\left(\mathbf{N}_{3}\right)$ & In vitro optical imaging & 197 \\
\hline & & Rituximab (DBCO) & Dendrimers $\left(\mathbf{N}_{3}\right)$ & In vivo imaging and therapy & 198 \\
\hline & & Metabolic lipid $\left(\mathbf{N}_{3}\right)$ & RBCG (DBCO) & In vivo therapy & 199 \\
\hline & \multirow[t]{2}{*}{$\mathrm{BCN} / \mathrm{N}_{3}$} & $\mathrm{Ac}_{4} \mathrm{MAnNAz}\left(\mathbf{N}_{3}\right)$ & Chitosan NPs (BCN) & $\begin{array}{l}\text { In vivo optical imaging, } \mathrm{MR} \text { and } \\
\mathrm{CT} \text { imaging }\end{array}$ & 200 \\
\hline & & $\mathrm{Ac}_{4} \mathrm{MAnNAz}\left(\mathbf{N}_{3}\right)$ & Au NPs (BCN) & In cellulo imaging & 201 \\
\hline \multirow{3}{*}{ 哭 } & \multirow{3}{*}{$\mathrm{Tz} / \mathrm{TCO}$} & $\begin{array}{l}\text { mAbs (anti-HER-2, anti-EGFR and } \\
\text { anti-EpCAM) (TCO) }\end{array}$ & $\begin{array}{l}\text { MFNPs (Tz) } \\
\text { QDs (Tz) }\end{array}$ & $\begin{array}{l}\text { in vitro fluorescence imaging } \\
\text { In vitro detection of intracellular } \\
\text { biomarkers }\end{array}$ & $\begin{array}{l}202 \\
203\end{array}$ \\
\hline & & $\begin{array}{l}\text { Antibiotics (Vancomycin and } \\
\text { Daptomycin) (TCO) }\end{array}$ & MFNPs (Tz) & $\begin{array}{l}\mu N M R \text { detection of Gram- } \\
\text { positive bacteria/Bactericidal } \\
\text { treatment }\end{array}$ & 204 \\
\hline & & Crystal violet (TCO) & MFNPs (Tz) & $\begin{array}{l}\text { Detection of Gram-positive } \\
\text { bacteria }\end{array}$ & \\
\hline
\end{tabular}




\begin{tabular}{|c|c|c|c|c|}
\hline & $\begin{array}{l}\text { Antigen-glutathione-S-transferase } \\
\text { (GST) (TCO/Tz) }\end{array}$ & NPs $(\mathrm{TCO} / \mathrm{Tz})$ & $\begin{array}{l}\text { SPR sensor chip (interaction, } \\
\text { kinetics and functionalization } \\
\text { studies) }\end{array}$ & 206 \\
\hline & $\begin{array}{l}\text { mAbs (anti-EGFR, Anti-EpCAM, } \\
\text { Anti-HER2 and Anti-MUC-1) (TCO) }\end{array}$ & MFNPs (Tz) & $\begin{array}{l}\mu \text { NMR detection of intracellular } \\
\text { cancer biomarkers / Profiling of } \\
\text { tumor cells }\end{array}$ & 207 \\
\hline & pHLIP (Tz) & HSA-NPs (TCO) & In vivo photothermal therapy & 208 \\
\hline & Trastuzumab (anti-HER2) (TCO) & SN38-Tz-NPs & $\begin{array}{l}\text { In vitro/In vivo drug delivery and } \\
\text { anticancer effect }\end{array}$ & 209 \\
\hline Tz/Norbornene & Anti-EGF (Tz) & QDs (Norbornene) & In vitro optical imaging & 54 \\
\hline
\end{tabular}

$\mathrm{N}_{3}$ : azide, DBCO: dibenzocyclooctyne, BCN: bicyclononyne, Tz: tetrazine, TCO: trans-cyclooctene, MFNPs: magneto-fluorescent nanoparticles, QDs: quantum dots, $\mathrm{Ac}_{4} \mathrm{MAnNAz}$ : tetraacetylated N-azidoacetyl-D-mannosamine, HSA: human serum albumin nanoparticles, pHLIP: low pH insertion peptide, DBCO : red blood cell ghosts.

\section{Examples of SPAAC}

SPAAC approaches have recently attracted increasing attention for enhancing the targeting ability without using biological targeting moieties such as mAbs. The metabolic glycoengineering method has been widely studied to incorporate bioorthogonal functional groups on cell surfaces of interest for further conjugation of NPs, fluorescent dyes or drugs. The functionalized synthetic sugars are usually specifically delivered to the target cells by $\mathrm{N}_{3^{-}}$ modified sugar molecules loaded in nanocarriers via the EPR effect or by direct intratumoral injection of these synthetic sugars.

\section{$\mathrm{DBCO} / \mathrm{N}_{3}$ cycloaddition}

In 2012, Koo et al. designed SPAAC click chemistry and metabolic glycoengineering for NPs to produce artificial glycan receptors. ${ }^{195}$ They selected tetraacetylated N-azidoacetyl-Dmannosamine ( $\left.\mathrm{Ac}_{4} \mathrm{ManNAz}\right)$, an unnatural sialic acid with $\mathrm{N}_{3}$ reactive groups, to generate targetable azido-glycans on cell surfaces specifically recognized by PEGylated liposomes modified with DBCO (DBCO-lipo) (Figure).

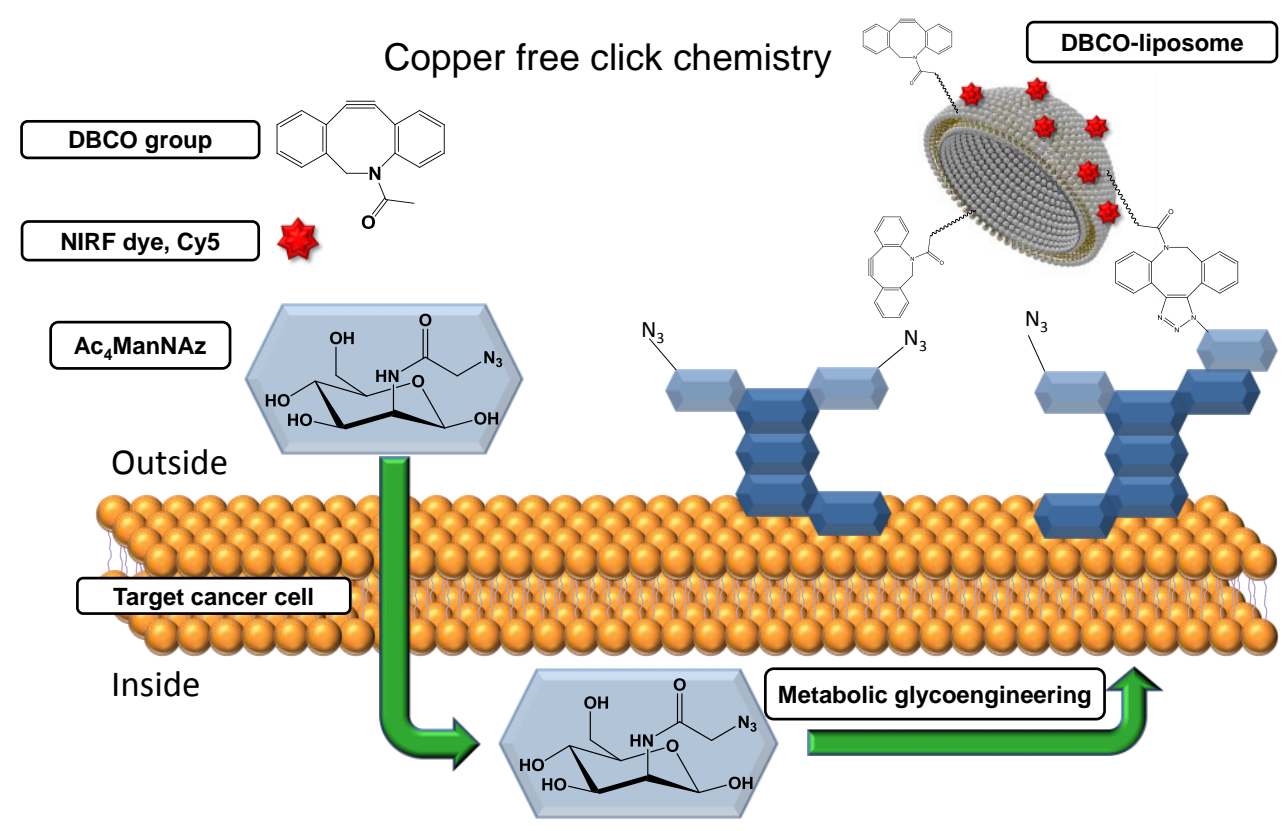

Figure 17. Schematic illustration of in vivo tumor-targeting strategy for DBCO-lipo nanoparticles by bioorthogonal copper-free click chemistry. Figure modified from ref ${ }^{195}$. 
Flow cytometry studies on $50 \mu \mathrm{M} \mathrm{Ac} 4$ ManNAz-treated A549 cells showed a 13.8-fold increase in cellular uptake of DBCO-lipo compared with PEG-lipo. These liposomes were intravenously administered and ex vivo tumor tissue analysis highlighted the advantages of the multivalency properties of the NPs (e.g. several reactive DBCOs per NP) and a longer circulation time in the blood to enhance tumor targeting compared with DBCO-Cy5 (NIRF intensity: $180 \%$ vs $6 \%$ ). They also confirmed the possibility of controlling the expression of these azide chemical precursors in a dose-dependent manner for sufficient delivery of DBCO-lipo. They observed tumor accumulation approximately 1.8 and 1.5 times higher in tumor-bearing mice treated with $50 \mathrm{mM}$ and $5 \mathrm{mM} \mathrm{Ac}_{4}$ ManNA, respectively. Moreover, this bioorthogonal based tumor-targeting strategy was validated in three other tumor cell lines expressing $\mathrm{Ac}_{4} \mathrm{MAnNAz}$ (U87MG, MCF-7 and KB).

In 2018, Meghani et al. developed clickable NPs for cancer therapy. ${ }^{45} \mathrm{Ac}_{4} \mathrm{MAnNAz}$ was used, as well as $\mathrm{N}_{3}$-sialic acid precursors specifically recognized by prior synthesized gelatin-oleicDBCO nanomaterials with embedded DOX (GON-DBCO-DOX). In vitro, GON-DBCO-DOX improved A549 and MCF-7 cell cytotoxicity compared with GON-DOX after 24 hours with 10 $\mu \mathrm{g} / \mathrm{mL}$ of DOX (for A549: $80 \%$ vs 50\%; for MCF-7: 70\% vs 50\%). Furthermore, confocal images after 4 hours of incubation demonstrated 2.4-fold and 4-fold higher cellular uptake on A549 and MCF-7 cells, respectively. A pretargeting strategy for QD- $\mathrm{N}_{3}$ able to bind cells metabolically modified with ManDBCO, an unnatural analog sugar of $\mathrm{Ac}_{4} \mathrm{ManDBCO}$, has also been reported. ${ }^{197}$ The cells were first labeled with DBCO, then QD- $\mathrm{N}_{3}$ was added as a secondary fluorescent imaging agent. Confocal images of ManDBCO-treated 4T1 cells showed specific and increasing cellular uptake (i.e. $30 \%$ at 2 hours vs $45 \%$ at 4 hours) compared with no cellular uptake for cells only treated with $\mathrm{QD}-\mathrm{N}_{3}$.

A pretargeted nanoradioimmunotherapy strategy was investigated by Au et al. for the specific delivery of radiolabeled PAMAM dendrimers for a targeting and therapeutic effect on NonHodgkin Lymphoma (NHL). ${ }^{198}$ The authors expected to deposit large amounts of ionizing radiation in the tumor site to achieve a high rate of tumor cell death while minimizing the effects on normal tissues. Rituximab, an anti-CD20 antibody ( $\alpha$-CD20) commonly used in $\mathrm{NHL}$ immunotherapy, was functionalized with DBCO as described in

Figure.

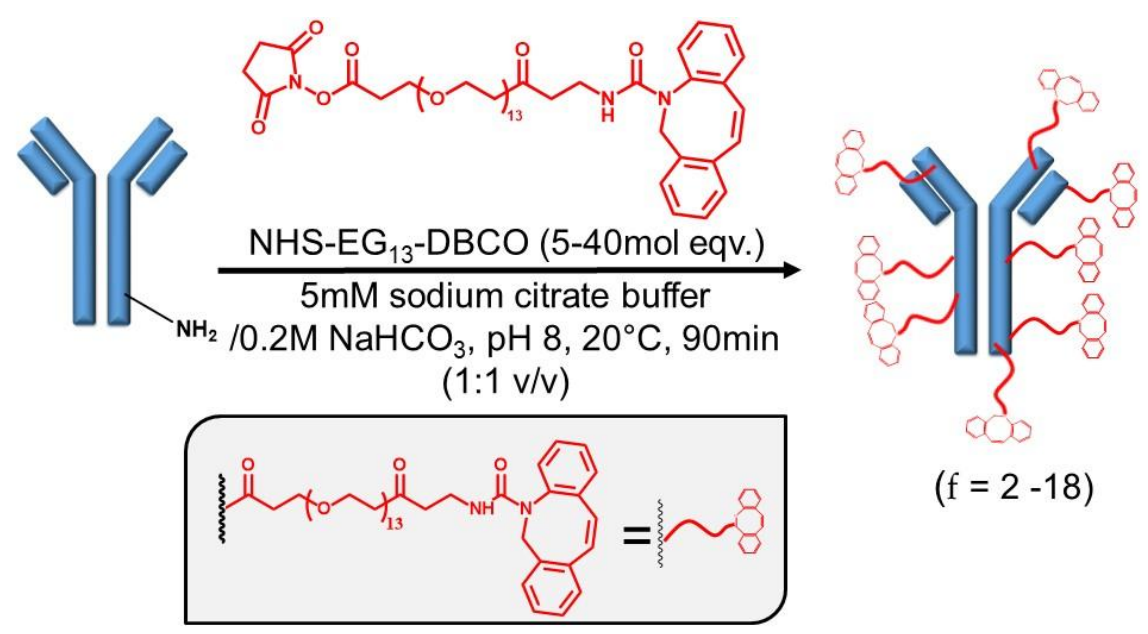

Figure 18. Functionalization of the primary targeting agent rituximab ( $\alpha-C D 20)$ with DBCO. ( $f$ : number of functions). Reprinted adapted with permission from ref ${ }^{198}$. Copyrright 2018 American Chemical Society. 
The pretargeting strategy consisted of injecting intravenously the DBCO-Rituximab conjugate in the first step. After 24 hours, $\mathrm{N}_{3}-\mathrm{Y}-90$ dual-modified dendrimers were administered to react with the previously injected modified mAb. Different amounts of $\operatorname{DBCO}(2,5,10,15$ and 18 $\mathrm{DBCO}$ per $\mathrm{mAb}$ ) were conjugated per $\alpha$-CD20): the $\alpha$-CD20 grafted with 10 DBCO (i.e. $\alpha$ CD20(DBCO $)_{10}$ ) was found to be the most appropriate to react with the $\mathrm{N}_{3}$-dendrimers. However, the dendrimer conjugated with $29 \mathrm{~N}_{3}$-oligoethylene glycol (i.e. PAMAM D$\left.\left({ }^{90} \mathrm{Y}\right)_{8}(\mathrm{~N})_{29}\right)$ was selected for its ability to form hyper-cross-links with $\alpha$-CD20(DBCO) ${ }_{10}$, thus improving activation of the complement-dependent cytotoxicity (CDC). The bioorthogonal reaction between PAMAM D- $\left({ }^{90} \mathrm{Y}\right)_{8}(\mathrm{~N})_{29}$ and the DBCO-labeled Raji cells was confirmed using both confocal microscopy and flow cytometry, with MFI almost twice as high as with the direct labeling strategy (117 vs 68). An apoptosis study also showed 10-fold higher therapeutic efficiency with the pretargeted approach compared with direct labeling; and $\beta^{-}$ radiation demonstrated 3.3-fold higher inhibition of cell proliferation.

In vivo experiments performed on Raji tumor-bearing mice revealed a significantly higher rate of DNA damage associated with a $17 \%$ increase of the survival median compared with $10 \%$ for clinical Rituximab and $\alpha$-CD20(DBCO) ${ }_{10}$ alone. Furthermore, tumor growth decreased for approximately $67 \%$ of the treated mice. In vivo therapeutic studies on a more aggressive disseminated lymphoma model confirmed higher tumor growth inhibition over time compared with the direct labeling method (46 days vs 25 days).

\section{$\mathrm{BCN} / \mathrm{N}_{3}$ cycloaddition}

In 2017, Lee et al. also generated artificial $\mathrm{N}_{3}$-glycans $\left(\mathrm{Ac}_{4} \mathrm{MAnNAz}\right)$ to deliver bicyclononyne-functionalized chitosan NPs (BCN-CNPs) to human mesenchymal stem cells (hMSCs). They hoped to enable stem cell imaging using optical imaging (BCN-CNP-Cy5.5), $\mathrm{MR}$ and CT imaging techniques (BCN-CNP-IRON and BCN-CNP-GOLD). ${ }^{200}$ In vitro, confocal images showed higher cellular binding for $\mathrm{Ac}_{4} \mathrm{MAnNAz} / \mathrm{BCN}-\mathrm{CNP}-\mathrm{Cy} 5.5$ with $99 \%$ labeled $v s$ $31.6 \%$ for BCN-CNP-Cy5.5-treated stem cells. In vivo optical imaging confirmed SPAAC between $\mathrm{N}_{3}$ groups on the cell surface and BCN with a NIRF intensity 15 times higher than with BCN-CNP-Cy5.5 alone. Moreover, the imaging signal of hMSCs remained for up to 15 days after transplantation compared with 5 days for BCN-CNP-Cy5.5. Finally, MR and CT images confirmed these observations with 5.4-fold and 2.5-fold higher signals, respectively.

\section{Examples of IEDDA}

\section{Tetrazine/TCO cycloaddition}

Among bioorthogonal reactions, the Tz/TCO reaction is well documented for the two-step NP delivery strategy. Most publications report the use of TCO-mAbs for the first step to enable specific cell recognition then to facilitate NP attachment for imaging, diagnostic or drug delivery purposes.

In 2010, Weissleder and co-workers named this approach 'bioorthogonal NP detection' (BOND) and more precisely two-step BOND (BOND-2) (Figure). ${ }^{202}$ In their study, they designed Tz-magneto-fluorescent NPs (Tz-MFNPs) and tested their targeting ability on extracellular cancer cell receptors using mAbs (anti-HER-2, anti-EGFR and anti-EpCAM) containing TCO (TCO-mAbs). They compared this BOND-2 method, in which the cells were first incubated with TCO-mAbs and then with MFNPs, with BOND-1, which consisted in direct labeling of the mAbs on the NPs before cell contact. They found BOND-2 to be up to 15 
times superior to the mAb-MFNP method, resulting in a strong fluorescence signal at the cell membranes. This amplification can be explained by the small size and high valencies (up to $30 \mathrm{TCO} / \mathrm{mAb}$ ) of the bioorthogonal functions, allowing covalent binding of several NPs with each mAb. They applied this two-step strategy using avidin/biotin interaction and demonstrated signals approximately twice as low as with BOND-2 on HER-2 and EpCAM cells. Steric hindrance of avidin $(66-\mathrm{kDa})$ and a lower valency than Tz-MFNPs (8 biotins vs $84 \mathrm{Tz}$, respectively) could explain these results. They successfully adapted the strategy with the same Tz-MFNPs, in situ, to detect intracellular biomarkers on previously fixed and permeabilized cells using TCO-anti-CK Ab (SK-OV-3, HeLa, SK-BR-3 and PANC-1) or TCOanti-Ki-67 Ab (S-KOV-3, SK-BR-3, A549, HT-29 and PANC-1). ${ }^{203}$ Fluorescent images were also obtained with Tz-QDs and TCO-anti-CK Ab or TCO-anti-Ki-67 Ab on PANC-1 cells.

\section{(a) One-step BOND (BOND-1)}

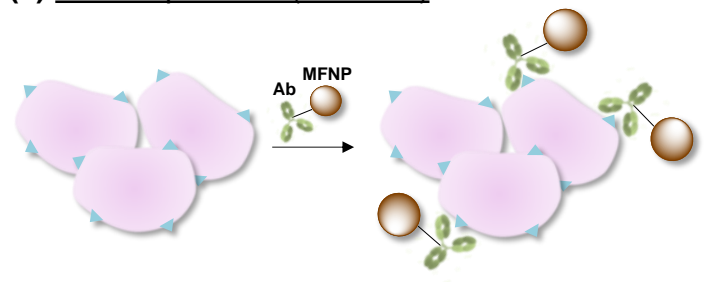

(b) Two-step BOND (BOND-2)

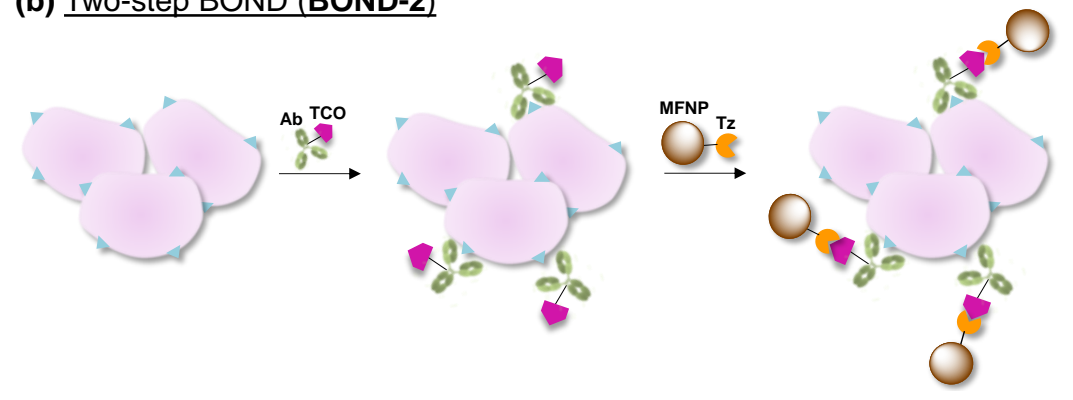

Figure 19. Application of the BOND strategy for one-step (direct, BOND-1) and two-step (bioorthogonal amplification, BOND-2) targeting of MFNPs to cells. Figure modified from ${ }^{202}$.

In 2014, pretargeting NPs were used to detect intracellular cancer biomarkers in human cells. Ghazani and his team used TCO-mAbs and Tz-MFNPs to profile lung tumor cells using $\mu$ NMR technology. ${ }^{207}$ Fine needle aspiration (FNA) and peripheral blood samples from routine biopsies were obtained from a cohort of 35 patients. The four biomarkers previously identified (EGFR, EpCAM, HER-2 and MUC-1) established the type of lung malignancy. Concordance of cancer biomarkers between circulating tumor cells (CTC) and FNA was demonstrated as well as better diagnostic accuracy compared with conventional histopathology.

More recently, Yoo et al. transposed this strategy by using TCO-modified trastuzumab (TCOTrb), which can bind HER2-overexpressed NIH3T6.7 cells, and tetrazine-linked NPs (TzNPs), including chemotherapeutic SN38. ${ }^{209}$ Using fluorescence microscopy, they demonstrated in vitro that Trb treated with 300 eq. increased Tz-NP binding and induced a subsequent effective reduction of cell viability (up to $45 \%$ for cells treated with a concentration of $4 \mu \mathrm{g} / \mathrm{mL}$ of SN38). In agreement with these in vitro results, the in vivo biodistribution of NPs intravenously injected 12 hours after TCO-Trb administration produced NIRF signals twice as high as those found in tumor-bearing mice that did not receive the TCO-Trb injection. 
Another two-step tumor-targeting strategy based on artificial chemical receptor expression on the tumor cell surface is also described. ${ }^{208} \mathrm{Tz}$ was conjugated with a low $\mathrm{pH}$ insertion peptide (pHLIP) known to cross the membrane bilayer in acidic conditions. The Tz-derivated pHLIP ( $p T z)$ was successfully anchored to HeLa cells, vascular endothelial cells (VEC) and tumorassociated fibroblasts (TAF). TCO-conjugated human serum albumin NPs were synthesized and modified with indocyanine green (TCO-HSA-ICG NPs = THI) enable specific pTz binding. In vivo studies on mice bearing HeLa tumors showed 2.6-fold and 1.7-fold higher tumor accumulation with $\mathrm{pTz} / \mathrm{THI}$ compared with $\mathrm{THI}$ and direct active targeting HSA-ICG NPs modified with FA, respectively. Photothermal therapeutic efficiency was higher, with a $100 \%$ survival rate after 50 days and negligible tumor relapse.

Several investigations have involved the detection of different gram-positive bacteria. ${ }^{204}$ In 2011, vancomycin and daptomycin antibiotics were modified with TCO (i.e. vanc-TCO and dapt-TCO) for subsequent bioorthogonal labeling with Tz-MFNPs. These antibiotics were able to bind with the specific peptidoglycan layer of gram-positive bacteria and inhibit cell wall synthesis. Micro-nuclear magnetic resonance system ( $\mu$ NMR) studies revealed up to 6fold higher bacterial targeting on Staphylococcus aureus compared with direct conjugates. These results reflect the ability of the chemically modified antibiotics to preserve their bactericidal activity. PT has also been successfully applied to the intracellular detection of bacteria in live macrophages in combination with permeabilizing agents.

In 2012, this technique was extended to detect and classify bacteria by $\mu N M R$ and optical imaging using a crystal violet modified with TCO (CV-TCO) and Tz-MFNPs. ${ }^{205}$ Tassa et al. reported Tz/TCO cycloaddition to produce sensor chips using Surface Plasmon Resonance (SPR). ${ }^{206}$ This system is based on the specific recognition of mAbs prior to immobilization on the gold layer of the SPR sensor surface with antigen-glutathione-S-transferase (GST) modified with TCO or Tz. Modifications allowed the subsequent bioorthogonal reaction with Tz derivatives or TCO-NPs, respectively. This sensor chip achieved successful interaction and good performance in kinetic studies and also functionalized NPs for molecular imaging using the rapid and highly specific IEDDA cycloaddition reaction.

\section{Tz/norbornene cycloaddition}

In 2010, Han et al. developed norbornene-coated QDs for live cell imaging. ${ }^{54}$ The reaction between Tz-Alexa 594 and norbornene-QDs incorporated approximately 16 norbornenes per QD. This strategy was applied for the targeting of A431 human carcinoma cells after functionalization of the epidermal growth factor (EGF) with Tz (Tz-EGF). The fluorescence intensity observed on cells for in situ conjugation (Tz-EGF/norbornene-QD) was higher than with direct labelling (QD-EGF).

\section{Other reactions}

PT based on non-covalent interaction between large structures has also been reported recently. Supramolecular binding, such as the 'host-guest' interaction, is known to have a faster reaction rate (i.e. $k_{2}=10^{9} \mathrm{M}^{-1} \mathrm{~s}^{-1}$ ) than traditional bioorthogonal covalent reactions (i.e. $\left.k_{2}=1-10^{4} \mathrm{M}^{-1} \mathrm{~s}^{-1}\right) .{ }^{210}$ Some mAb targeting vectors (e.g. anti-HER-2, anti-EGFR and antiEpCAM) functionalized with $\beta$-cyclodextrin (CD) have been used with adamantane-MFNPs as a secondary imaging agent for profiling cancer cells. This study demonstrated, in vitro, that this kind of labeling was 15 times superior to the direct conjugation method (mAbMFNPs) and twice as effective as the non-covalent avidin-biotin system. The labeling 
protocol was finally assessed using QDs and magnetic beads demonstrating highly specific interactions; one possible application would be the separation of HER-2-positive and negative cells by a magnetic sorting process.

Covalent oxime click chemistry is described in another publication for the selective and efficient delivery of nucleic acids to cells. ${ }^{211}$ The cell surface was first engineered with ketone by rapid fusion of ketone-functionalized liposomes. The cells expressing ketones were then specifically recognized by adding oxyamine/nucleic acid lipoplex, which was subsequently internalized and released for transfection in the cells. Using flow chemistry, the in vitro study found that the number of ketone groups expressed on the cell surface was dependent on the keto-liposome exposure time. This system presented higher selectivity and efficiency on fibroblast cells compared with conventional reagent transfection (i.e. $68 \%$ vs $29 \%$ and $19 \%$ for Viafect and Lipofectamine 3000, respectively).

\subsection{PT approaches with NPs as a "platform"}

Recent studies on PT approaches using NPs as a "platform" are summarized in Table 6.

Table 6. General overview of PT approaches using NPs as a "platform".

\begin{tabular}{|c|c|c|c|c|c|}
\hline & $\begin{array}{l}\text { Bioorthogonal } \\
\text { system }\end{array}$ & $\begin{array}{l}\text { Primary tumor-targeting agent } \\
\text { for the first step of the PT } \\
\text { approach }\end{array}$ & $\begin{array}{l}\text { Complementary agent for the } \\
\text { second step of the PT } \\
\text { approach }\end{array}$ & Application & Refs. \\
\hline \multirow{7}{*}{$\frac{ن}{4}$} & \multirow{5}{*}{$\mathrm{DBCO} / \mathrm{N}_{3}$} & $\mathrm{Ac}_{4} \mathrm{MAnNAz}$-liposomes $\left(\mathbf{N}_{3}\right)$ & $\begin{array}{l}\text { Zinc(II)-phtalocyanine liposomes } \\
\text { (DBCO) }\end{array}$ & $\begin{array}{l}\text { In vitro/in vivo photothermal, } \\
\text { photodynamic, photoacoustic } \\
\text { imaging and therapy }\end{array}$ & 212 \\
\hline & & $\begin{array}{l}\text { MIL-100 (Fe) NPs embedding 3- } \\
\text { azido-D-alanine }\left(\mathbf{N}_{3}\right)\end{array}$ & $\begin{array}{l}\text { Ultrasmall photosensitizer NPs } \\
\text { embedding TPETM molecules } \\
\text { (DBCO) }\end{array}$ & $\begin{array}{l}\text { In vivo imaging and PDT of } \\
\text { bacteria }\end{array}$ & 213 \\
\hline & & MSNs (DBCO) & ${ }^{18} \mathrm{~F}\left(\mathbf{N}_{\mathbf{3}}\right)$ & In vivo $\mathrm{PET}$ imaging & 214 \\
\hline & & MSNs-RAW (DBCO) & ${ }^{18} \mathrm{~F}\left(\mathbf{N}_{3}\right)$ & In vivo $\mathrm{PET}-\mathrm{CT}$ imaging & 215 \\
\hline & & Ac4MAnNAz-PEG-PLA NPs & DBCO-Ce6 & In vivo photodynamic therapy & 216 \\
\hline & \multirow{2}{*}{$\mathrm{BCN} / \mathrm{N}_{3}$} & $\mathrm{Ac}_{4} \mathrm{MAnNAz-chistosan} \mathrm{NPs}\left(\mathbf{N}_{3}\right)$ & Ce6-chitosan NPs (BCN) & In vivo photodynamic therapy & 217 \\
\hline & & $\mathrm{Ac}_{4} \mathrm{MAnNAz-}\left(\mathbf{N}_{3}\right)$ & $\mathrm{CNP}(\mathrm{BCN})$ & In vitro/in vivo optical imaging & 200,218 \\
\hline \multirow{4}{*}{$\begin{array}{l}\text { 吕 } \\
\text { 므 }\end{array}$} & \multirow{4}{*}{$\mathrm{Tz} / \mathrm{TCO}$} & ${ }^{89} \mathrm{Zr}$-liposomes (TCO) & PDA@CoCrMo (Tz) & $\begin{array}{l}\text { Specific removal of long- } \\
\text { circulating radiopharmaceuticals }\end{array}$ & 219 \\
\hline & & $\mathrm{TCO}-\mathrm{SiO}_{2} \mathrm{NPS}$ & ${ }^{11} \mathrm{C}(\mathbf{T z})$ & In vivolex vivo $\mathrm{PET}$ imaging & 220 \\
\hline & & SMNPs (TCO) & ${ }^{111}$ In-Tz-DOTA & $\begin{array}{l}\text { In vivo optical and SPECT } \\
\text { imaging }\end{array}$ & 221 \\
\hline & & Psi (TCO) & {$\left[{ }^{18} \mathrm{~F}\right]$ FDR-tetrazine $(\mathbf{T z})$} & In vivo $\mathrm{PET}$ imaging & 222 \\
\hline
\end{tabular}

$\mathrm{N}_{3}$ : azide, DBCO: dibenzocyclooctyne, BCN: bicyclononyne, Tz: tetrazine, TCO: trans-cyclooctene, $\mathrm{Ac}_{4} \mathrm{MAnNAz:}$ tetraacetylated Nazidoacetyl-D-mannosamine, TPETM: 2-(1-(5-(4-(1,2,2-tris(4-methoxyphenyl)vinyl)phenyl)thiophen-2-yl)ethylidene)malononitrile), MSNs: mesoporous silica nanoparticles, SMNPs: small molecule-based nanoparticles, PDA: polydopamine, CoCrMo: cobalt chromium molybdenum alloy, Psi : Mesoporous Silicon.

\section{Examples of SPAAC}




\section{$\mathrm{DBCO} / \mathrm{N}_{3}$ cycloaddition}

In 2017, Du et al. also investigated a two-step tumor-targeting strategy based on metabolic glycoengineering and SPAAC to deliver their multifunctional nanoagent to combine PTT, PDT, PA imaging and PAT (Figure20). ${ }^{212}$ First, $A_{4} M A n N A z$ was encapsulated by selfassembly in nanomicelles $\left(\mathrm{Ac}_{4} \mathrm{MAnNAz-LP}\right)$ to generate the artificial chemical-receptor in tumor cells, thanks to the EPR effect, after intravenous injection. DBCO-nanomicelles embedded near infrared dyes zinc(II)-phthalocyanine (DBCO-ZnPc-LP) were designed for both PTT and PA imaging. In vitro, PTT performed with a $0.4 \mathrm{~W} / \mathrm{cm}^{2}$ continuous laser on A549 cells showed higher cytotoxicity for $\mathrm{Ac}_{4} \mathrm{MAnNAz-LP/DBCO}-\mathrm{ZnPc}-\mathrm{LP}$ than DBCO-ZnPcLP alone $\left(80 \%\right.$ vs 35\%). Moreover, $\mathrm{Ac}_{4}$ MAnNAz-LP/DBCO-ZnPc-LP-treated cells demonstrated superior cytotoxicity with a combination of PTT/PAT compared with PTT and PAT alone with $63 \%, 22 \%$ and $20 \%$ cytotoxicity, respectively. PA imaging on in vivo experiments revealed 3-fold higher tumor uptake after 24 hours for $\mathrm{Ac}_{4} \mathrm{MAnNAz-LP/DBCO}$ ZnPc-LP compared with DBCO-ZnPC-LP. ICP-MS analyses showed that mice pre-treated with $\mathrm{Ac}_{4} \mathrm{MAnNAz-LP}$ for 3 days had a higher tumor uptake 6 hours after injection than no those with no pre-treatment $(6.30 \% \mathrm{IA} / \mathrm{g}$ vs $1.85 \% \mathrm{IA} / \mathrm{g})$, and tumor growth inhibition over time (18 days) with synergistic PTT/PAT.

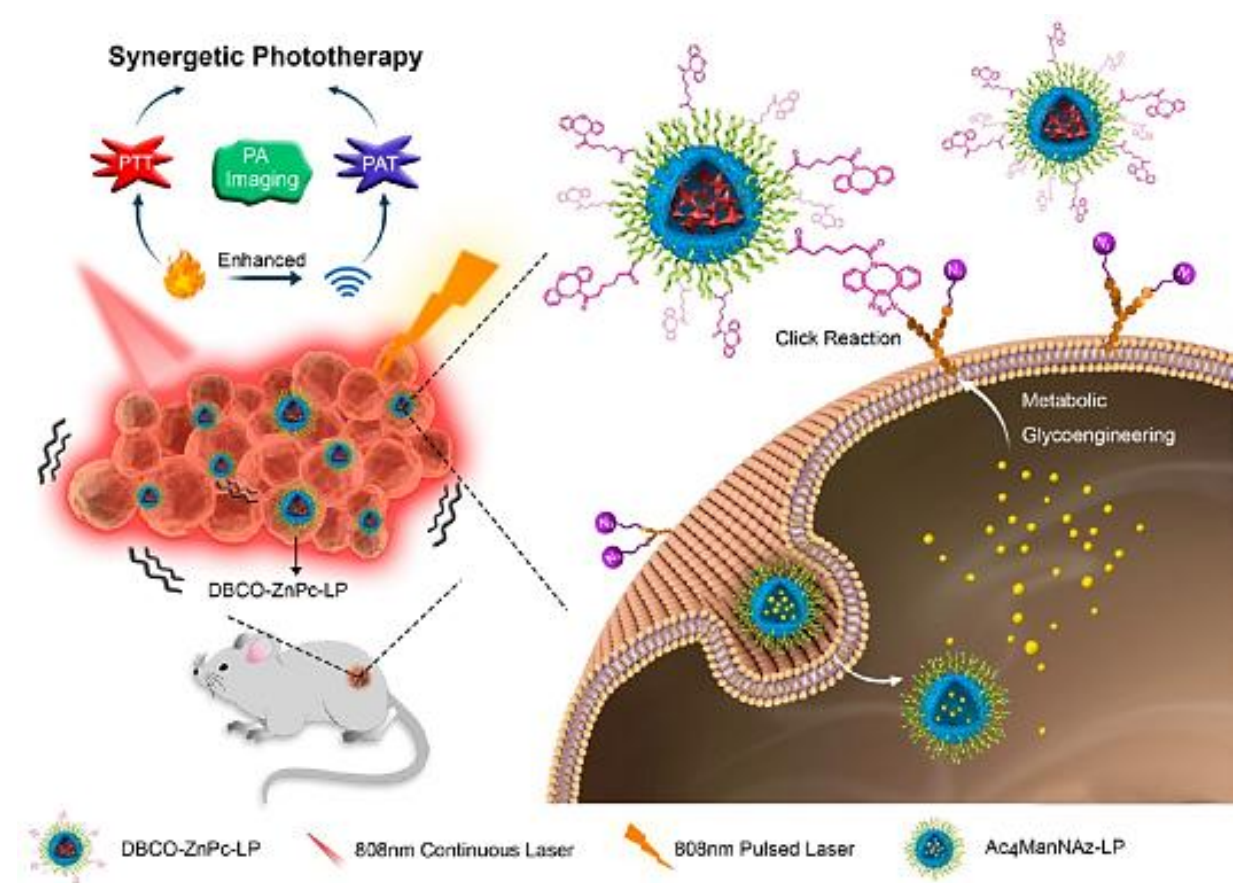

Figure 20. Schematic illustration of PA Imaging-Guided Synergistic PTT/PAT with the Bioorthogonal Metabolic Glycoengineering-Activated Tumor Targeting Nanoagent. Reprinted adapted with permission from ref ${ }^{212}$. Copyright 2017 American Chemical Society.

In 2018, Mao et al. performed specific in vivo imaging of bacteria and antibacterial treatment using clickable NPs. ${ }^{213}$ Two different NPs were administered for subsequent bioorthogonal conjugation. Firstly, MIL-100 (Fe) NPs encapsulating the 3-azido-D-alanine metabolic precursor (D-AzAla@MIL-100 (Fe)) were injected and accumulated in the infected regions of mice bearing methicillin-resistant Staphylococcus aureus (MRSA). These NPs decomposed in the presence of $\mathrm{H}_{2} \mathrm{O}_{2}$ secreted by the immune cells and released unnatural azido groups which were then metabolically expressed on MRSA bacterial walls. In the second step, ultrasmall DBCO-modified photosensitizer NPs integrating TPETM molecules (US-TPETM NPs) were administered for both imaging and photodynamic therapy (PDT). In vivo azido 
incorporation efficiency was evaluated using a DBCO-Cy5 fluorophore and demonstrated 3.2-fold higher fluorescence thanks to $\mathrm{H}_{2} \mathrm{O}_{2}$ responsiveness compared with normal tissues. In vivo, a stronger fluorescent signal was obtained after 24 hours in bacteria-bearing mice pre-treated with D-AzAla@MIL-100 (Fe) compared with mice only treated with US-TPETM NPs. The antibacterial effect was also significantly enhanced after white light irradiation for $10 \min (p<0.05)$.

In 2013, Lee et al. described the first preclinical pretargeting application of SPAAC in PET imaging. ${ }^{214}$ In situ formation of ${ }^{18} \mathrm{~F}$-DBCOT-PEG-MSNs thanks to the two-step strategy was reported for in vivo real-time tracking imaging (pharmacokinetic studies). DBCO-PEG-MSNs were first injected intravenously into U87MG tumor-bearing mice to encourage accumulation thanks to the EPR effect. 24 hours later, $\left[{ }^{18} \mathrm{~F}\right]$ fluoropentaethylene glycolic $N_{3}\left(\left[{ }^{18} \mathrm{~F}\right] 2\right)$ was administered and clicked specifically to the NPs within 2 hours. Biodistribution studies showed a significantly higher signal for DBCO-PEG-MSNs-pre-treated tumors (250 $\mu \mathrm{g}, 30$ $\mathrm{nmol} \mathrm{DBCO})$ compared with $\left[{ }^{18} \mathrm{~F}\right] 2$ alone, at $2.6 \mathrm{MBq}(1.4 \% \mathrm{IA} / \mathrm{g}$ vs $0.6 \% \mathrm{IA} / \mathrm{g})$.

A pretargeting PET imaging strategy was also assessed in 2019 for macrophage cell tracking in tumors and atherosclerosis plaques using ${ }^{18} \mathrm{~F}$ labeling to investigate the ability of the macrophages to accumulate in the affected areas. ${ }^{215}$ DBCO-MSN cells were thus incubated with RAW 264.7 macrophage cells for 2 hours at $37^{\circ} \mathrm{C}$ to obtain the desired DBCO-MSNRAW cells. These DBCO-MSN-RAW cells were then intravenously injected into U87MG tumor-bearing mice. Between one and eight days later, the mice were injected with $11.1 \mathrm{MBq}$ of ${ }^{18} \mathrm{~F}-\mathrm{N}_{3}$. PET-CT images demonstrated a larger accumulation of radioactivity at the tumorsite compared with ${ }^{18} \mathrm{~F}-\mathrm{N}_{3}$ alone $(3.8 \% \mathrm{IA} / \mathrm{g}$ vs $2.5 \% \mathrm{IA} / \mathrm{g})$. Finally, this specific binding and macrophage tracking method was also assessed on mice with atherosclerosis and showed similar efficient accumulation in the atherosclerotic aorta area.

\section{$\mathrm{BCN} / \mathrm{N}_{3}$ cycloaddition}

One study reports that the tumor accumulation of glycol chitosan NPs (CNPs) can be increased by metabolic glycoengineering. ${ }^{217}$ CNPs functionalized with $\mathrm{Ac}_{4} \mathrm{MAnNAz}$ $\left(\mathrm{Ac}_{4} \mathrm{MAnNAz-CNP}\right)$ by hydrophobic interaction were intravenously injected into mice bearing A549 tumors for accumulation thanks to the EPR effect. Other CNPs functionalized with BCN and chlorin e6 photosensitizers (BCN-Ce6-CNPs) were subsequently injected as secondary agents. In vitro, the pre-targeting strategy showed significantly higher cytotoxicity on A549 cells compared with the non-pre-treated BCN-Ce6-CNPs after laser irradiation $(90 \% \mathrm{vs}$ $40 \%$ ). In vivo, the biodistribution study revealed a significant 2-fold higher tumor uptake for $\mathrm{Ac}_{4} \mathrm{MAnNAz-CNP/BCN-Ce6-CNPs}$ compared with BCN-Ce6-CNPs. Moreover, efficient photodynamic therapy was observed 21 days after laser irradiation with no tumor relapse compared with BCN-Ce6-CNPs $(p<0.01)$, for which growth restarted after 4 days.

\section{Examples of IEDDA}

\section{Tetrazine/TCO cycloaddition}

In 2016, Denk et al. performed in vivo click experiments using TCO-MSNs and low-molecular weight ${ }^{11} \mathrm{C}$-tetrazine for PET imaging (Figure 21). ${ }^{220}$ They exploited the ability of these NPs to achieve rapid and exclusive accumulation in the lungs for the investigation of pretargeted PET imaging using bioorthogonal chemistry. TCO-MSNs were first administered for effective accumulation in lungs, before the ${ }^{11} \mathrm{C}-\mathrm{Tz}$ was injected five minutes later. Two-step protocol 
PET imaging demonstrated a 3-fold increase in activity concentration, and therefore in the gamma counter signal, compared with ${ }^{11} \mathrm{C}-\mathrm{Tz}$. In recent years, pretargeting NPs have been introduced as a suitable tool for nuclear imaging and radiotherapy. ${ }^{64}$

A

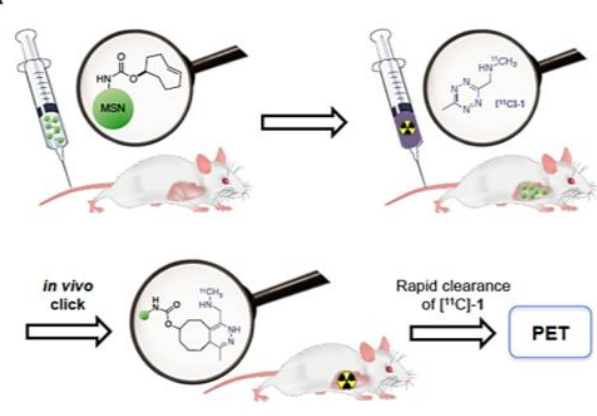

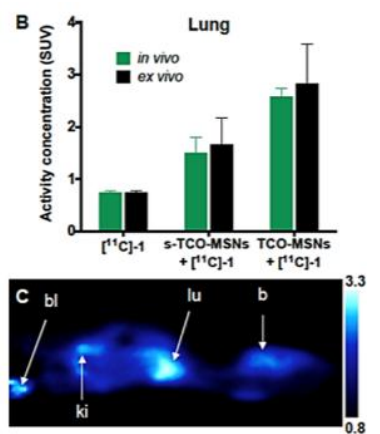

Figure 21. (A) In vivo click experiment using TCO-and s-TCO-modified silica nanoparticles (chemical structure shown for TCO). (B) Activity concentration measured in vivoby PET imaging (50-60 min) and ex vivo(after $60 \mathrm{~min}$, gamma counter) showing increased lung uptake in female BALC/c mice when using s-TCO-MSNs $(n=5)$ and TCO-MSNs $(n=2)$. (C) Pretargeted PET image (sagittal view, 40-60 min): TCO-MSNs + [11C]-1 (scale bar unit: SUV; $b=$ brain, $b l=$ bladder, $k=$ kidney, lu = lung. Reprinted adapted with permission from ref ${ }^{220}$. Copyright 2016 American Chemical Society.

In 2019, van Onzen et al. used IEDDA cycloaddition to radiolabel $\pi$-conjugated small molecule-based NPs (SMNPs) for both optical and SPECT imaging. ${ }^{221}$ The covalent conjugation between the ${ }^{111}$ In-radiolabeled Tz-DOTA and TCO-NPs was confirmed intrinsically. SMNPs were used as dual-imaging agents for biodistribution studies and accumulated in the liver and spleen (80.4\% IA/g and 34.8\% IA/g, respectively) 4 hours after injection but were efficiently cleared from the blood after $10 \mathrm{~min}(0.9 \% \mathrm{IA} / \mathrm{g})$. However, this imaging strategy is limited for long-life radionuclides (e.g. ${ }^{64} \mathrm{Cu}$ and ${ }^{89} \mathrm{Zr}$ ) which could deposit large amounts of radiation in non-targeted organs (e.g. liver and spleen).

To circumvent this problem, Brand et al. recently designed an IEDDA-based platform for the specific removal of long-circulating radiopharmaceuticals from the systemic circuit. ${ }^{219} \mathrm{Tz}-$ polydopamine-coated metal disks (Tz-PDA@CoCrMo) were developed to remove the TCO${ }^{89} \mathrm{Zr}$-radiolabeled liposomes $\left({ }^{89} \mathrm{Zr}\right.$-TCO-LNP) that remained in the blood after EPR accumulation in tumor cells. Phosphor autoradiography assays demonstrated 2-fold higher radioactivity on the disk for ${ }^{89} \mathrm{Zr}$-TCO-LNP compared with PDA@CoCrMo.

\section{Click chemistry for multifunctionalized NPs}

In the previous paragraphs of this manuscript, we have attempted to identify the potential roles of click chemistry in the field of nanoparticles. This early $20^{\text {th }}$ century chemistry offers a solution for encapsulating therapeutic agents, functionalizing imaging agents or therapeutic agents on the surface of NPs or grafting targeting agents to address NPs specifically according to the expression or overexpression of tumor receptors in order to amplify tumor uptake. Bioorthogonal chemistry (SPAAC or IEDDA) also provides a targeting approach based more specifically on monoclonal antibodies, probably due to the rapid development of anti-cancer immunotherapy over the last ten years. In this section, we present the opportunities offered by click chemistry for the development of multimodal NPs (nano-objects combining several imaging modalities: PET, SPECT, NIRF, MRI) or theranostic NPs (nanoobjects combining imaging and therapeutic agents). Among all the articles reviewed to establish this bibliographic analysis, we focused particularly on articles published since 2016 . 
Unlike the earlier part of this manuscript, the articles concerned are not classified by type of click chemistry but according to the multimodality of imaging and/or theranostics (Table 6). 
Table 7. General overview of multimodal or/and theranostic NP.

\begin{tabular}{|c|c|c|c|c|c|c|c|}
\hline $\begin{array}{c}\text { Multimodal } \\
\text { or/and } \\
\text { Theranostic NP }\end{array}$ & $\begin{array}{l}\text { Type } \\
\text { of NP }\end{array}$ & $\begin{array}{c}\text { Type of } \\
\text { click chemistry }\end{array}$ & Type of imaging & $\begin{array}{c}\text { Therapeutic } \\
\text { approach }\end{array}$ & Passive or Active targeting & $\begin{array}{c}\text { In vitro/In vivo } \\
\text { studies }\end{array}$ & Refs. \\
\hline \multirow{5}{*}{ Multimodal } & Glycol chitosan NP & $\mathrm{N}_{3} / \mathrm{DBCO}$ & FITC/PET & & Passive & In vivo & 108 \\
\hline & Liposome & $\mathrm{N}_{3} / \mathrm{DBCO}$ & FITC/PET & & Passive & In vivo & 109 \\
\hline & Iron nanoparticle & $\mathrm{N}_{3} /$ alkyne & MRI/HIR & & Active & In vitro & 132 \\
\hline & Porous silica NP & $\mathrm{N}_{3} / \mathrm{DBCO}$ & FITC/PET & & Active & In vitrolin vivo & 134 \\
\hline & Glycol chitosan NP & $\mathrm{N}_{3} / \mathrm{BCN}$ & FITC/MRI & & Active & In vitro/in vivo & 217 \\
\hline \multirow{8}{*}{ Theranostic } & Liposome & $\mathrm{N}_{3} / \mathrm{DBCO}$ & FITC/PA & PTT & Active & In vivo & 212 \\
\hline & Glycol chitosan NP & $\mathrm{N}_{3} / \mathrm{BCN}$ & FITC & PDT & Active & In vitro/in vivo & 217 \\
\hline & HSA-NP (albumine) & $\mathrm{Tz} / \mathrm{TCO}$ & FITC & PTT & Active & In vitro/in vivo & 208 \\
\hline & & & & & & & \\
\hline & Liposome & $\mathrm{N}_{3} / \mathrm{BCN}$ & FITC & Drug carrier & Passive & In vitro/in vivo & 229 \\
\hline & Liposome & Tz/vinyl ether & FITC & PTT/Drug carrier & Passive & In vitrolin vivo & 231 \\
\hline & LQ /DOX-ZnPc nanocomposite & $\mathrm{N}_{3} / \mathrm{DBCO}$ & & PTT/Drug carrier & Active & In vitrolin vivo & 223 \\
\hline & Porous silica NP & $\mathrm{N}_{3} / \mathrm{DBCO}$ & FITC/SPECT & Drug carrier & Active & In vitro/in vivo & 224 \\
\hline \multirow[t]{2}{*}{ Multimodal/Theranostic } & $\begin{array}{l}\text { Ac4MAnNAz-PEG-PLA NPs } \\
\text { + DBCO-PEG-PAEAM NPs }\end{array}$ & & MRI & PDT & Active & In vitrolin vivo & 225 \\
\hline & Liposome & & NIRF & PTT & Active & & 226 \\
\hline
\end{tabular}




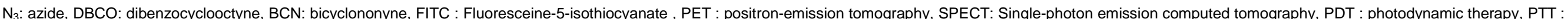
photothermal therapy, LQ : (LMWH)-quercetin (Qu) conjugate, DOX : doxorubicin, ZnPc : zinc phthalocyanine, PAEAM : poly(2-azepane ethyl methacrylate), NIRF : Near infrared fluorescence imaging 


\subsection{NPs using click chemistry for multimodal imaging}

As indicated by Lee et al., ${ }^{108}$ imaging techniques, such as computed tomography, magnetic resonance imaging, and positron emission tomography (PET), offer many potential benefits for the diagnosis and treatment of cancers. Each method has its own strengths and weaknesses. Multimodal imaging techniques have thus been pinpointed as an alternative method to overcome the limitations of each individual imaging technique. The authors also developed a dual optical/PET imaging CNP (glycol chitosan nanoparticle). The azido group available on the surface of the NP allows grafting of the 64Cu-radiolabeled DOTA complex and the activatable MMP-probe via copper-free click chemistry (Figure ). To validate the use of dual PET/activatable NIRF probe-labeled CNPs for tracking nanoparticles and imaging the tumor in vivo, whole-body NIRF and PET images were obtained from A549 tumor-bearing mice after intravenous injection of AMP-CNP-DOTA $-{ }^{64} \mathrm{Cu}$. The PET images revealed that the accumulation of AMP-CNP-DOTA- ${ }^{64} \mathrm{Cu}$ in the tumor increased gradually with time, reaching a plateau 24 hours after injection (5\% ID/g). To confirm these in vivo multimodal imaging observations, the NIRF signals of the tumor and other major organs were quantified and the highest NIRF signal intensity was found in the tumor region.

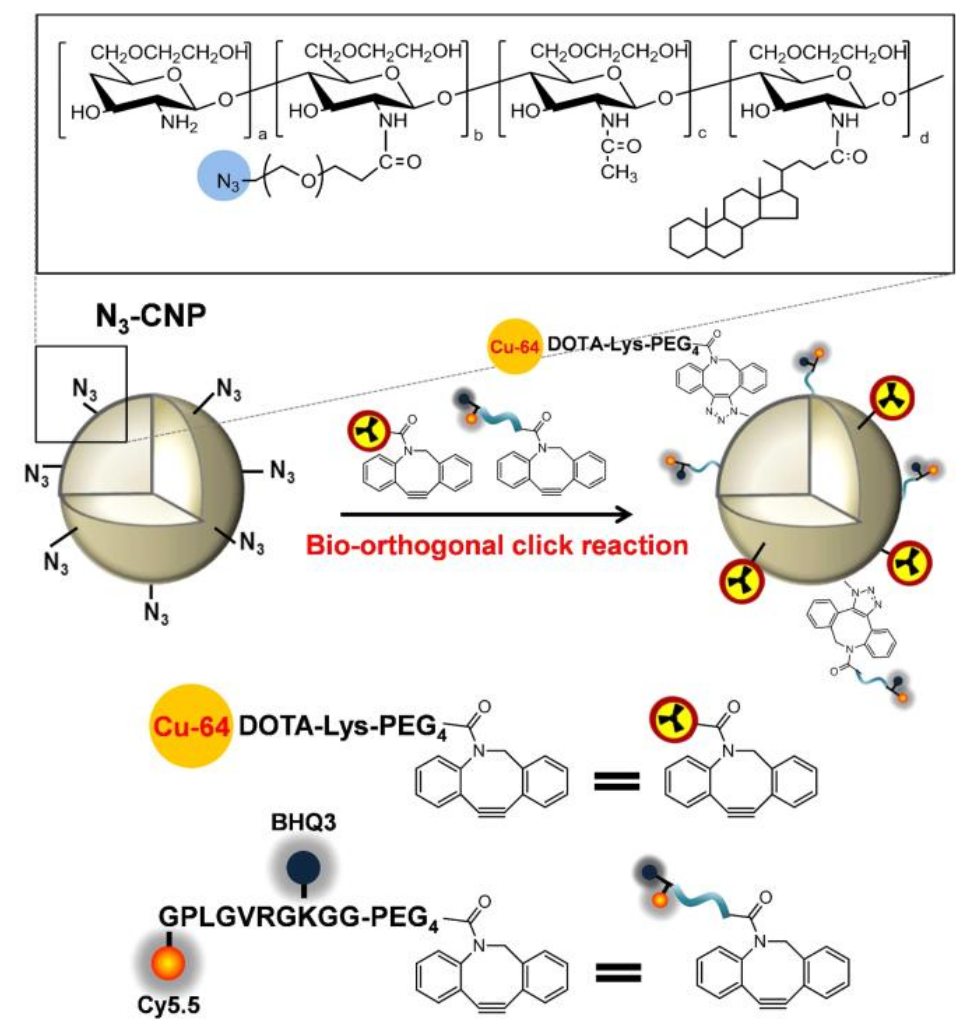

Figure 22. Chemical structure of azide-functionalized glycol chitosan-5 $\beta$-cholanic acid conjugate $\left(C N P-N_{3}\right)$ and schematic illustration for the labelling of DOTA-Lys-PEG 4 -DBCO with ${ }^{64} \mathrm{CU}$ and AMP-DBCO (AMP-DBCO is composed of MMP-specific peptide, NIRF dye (Cy5.5), dark quencher (BHQ-3), and DBCO) onto azidefunctionalized CNP via bio-orthogonal click chemistry. Reprinted with permission from ref ${ }^{108}$. Copyright 2014 American Chemical Society.

In a similar approach, Perez-Medina et al. developed a dual-modality liposomal nanoparticle combining a PET label and a near-infrared (NIR) fluorophore, bringing together the best of both worlds, i.e. the high sensitivity and tissue penetration of PET and the cellular resolution of optical imaging. ${ }^{227}$ In this study, copper-free click chemistry was used to graft the labelling 
${ }^{89} \mathrm{Zr}$ probe. The fluorescent dyes were incorporated into the lipid bilayer of the liposomal NPs. In vivo biodistribution of the ${ }^{89} \mathrm{Zr}$-CLL was compared with that of ${ }^{89} \mathrm{Zr}$-SCL in mice bearing 4T1 breast cancer xenografts (for the latter, no click chemistry was used and the radionuclide was chelated by DFO grafted onto the surface of the NPs). However, no ${ }^{89} \mathrm{Zr}$-CLL signal was detected in the tumor, unlike the ${ }^{89} \mathrm{Zr}$-SCL (up to $24 \% \mathrm{ID} / \mathrm{g}$ in the tumor).

Yuan et al. used the powerful click chemistry (SPAAC) method to generate multifunctional nanomaterials to allow MRI detection of NPs (superparamagnetism of iron NPs), fluorescence (click attachment of fluorochromes), and radioactivity. ${ }^{132}$ The click chemistry group available on the surface of the NP thus allows the grafting of different fluorescent probes as well as the association of targeting agents (folate, RGD or protamine). This multimodal approach to nanoparticles, which can be surface functionalized by different targeting agents using click chemistry, could enable the design of a "universal" nano-object, suitable for a range of cancer pathologies. This nanoparticle has only been evaluated in vitro for some targeting agents.

Wang et al. developed azido-modified silica nanoconjugates (azido-/Cy5-NCs) that not only enabled dual-modal PET/CT and fluorescence imaging but could also target the cell-surface DBCO groups via SPAAC click chemistry. ${ }^{134}$ Azido and DOTA (for radiolabeling with ${ }^{64} \mathrm{Cu}$ ) groups were grafted onto the surface of NCs and fluorescent dyes were encapsulated inside. For this in vivo study, LS174T tumors were developed in athymic nude mice by subcutaneous injection of LS174T cells into both flanks, then $\mathrm{Ac}_{4} \mathrm{ManDBCO}$ moieties (5 $\mathrm{mg} / \mathrm{kg}$ ) were injected intratumorally (i.t.). $\mathrm{Ac}_{4} \mathrm{ManDBCO}$ can be incorporated into the cell membrane surface, thus creating "artificial tumor receptors". The combination of the $\mathrm{Ac}_{4}$ ManDBCO system and click chemistry demonstrated advantages in terms of high targeting efficiency, absence of immunogenicity, and easy manufacture. ${ }^{64} \mathrm{Cu}$-/azido-/Cy5$\mathrm{NCs}$ or ${ }^{64} \mathrm{Cu}-/ \mathrm{Cy} 5-\mathrm{NCs}$ were injected intravenously and biodistribution was monitored by PET/CT imaging. Measurement of the radioactivity of the tissues harvested 24 hours after injection showed a 1.7-fold accumulation of ${ }^{64} \mathrm{Cu}$-/azido-/Cy5-NCs in the tumors on the left pretreated with Ac4ManDBCO compared with the tumors on the right that were not pretreated with glycoengineering sugar. However, the difference between the left- and righthand tumors was negligible in terms of accumulation of ${ }^{64} \mathrm{Cu}-/ \mathrm{Cy} 5-\mathrm{NCs}$ without azido modification. Moreover, confocal imaging of tissue sections of $\mathrm{Ac}_{4} \mathrm{ManDBCO}$-treated tumors showed a noticeable amount of azido-/Cy5-NCs accumulated in tumors 6 hours after injection, and a clear Cy5 fluorescence contrast was observed between the tumors on the left pretreated with $\mathrm{Ac}_{4} \mathrm{ManDBCO}$ and the tumors on the right pretreated with PBS.

In 2017, Lee et al. also investigated this glycoengineering system combined with modified glycol chitosan nanoparticles (BCN-CNPs) to deliver different imaging agents: Cy5.5, iron oxide NPs and gold NPs were conjugated with or encapsulated into BCN-CNPs for optical, $\mathrm{MRI}$ and $\mathrm{CT}$ imaging. ${ }^{200}$ They started by testing three different glycoengineering systems $\left(\mathrm{Ac}_{4} \mathrm{ManN}_{3}, \mathrm{Ac}_{4} \mathrm{GaIN}_{3}\right.$ and $\left.\mathrm{Ac}_{4} \mathrm{GlcN}_{3}\right)$. Importantly, $\mathrm{Ac}_{4} \mathrm{ManN}_{3}$-treated hMSCs produced a significantly higher signal in membrane extract compared with other hMSCs treated with $\mathrm{Ac}_{4} \mathrm{GaIN}_{3}$ or $\mathrm{Ac}_{4} \mathrm{GlcN}_{3}$. To evaluate this imaging method to target chemical receptors, they then tested the imaging efficiency (MR and $C T$ ) of BCN-CNPs containing iron oxide nanoparticles (BCN-CNP-IRON) or gold nanoparticles (BCN-CNP-GOLD) to track stem cells in live animals. Approximately $100 \%$ of the cells were labeled with each nanoparticle, showing positive signals for both imaging techniques. 


\subsection{NPs using click chemistry for a theranostic approach}

The two-step tumor-targeting strategy based on metabolic glycoengineering and click chemistry, an emerging tumor-targeting technique that has already demonstrated high tumorspecificity with a remarkable advantage over biological receptors, has also been used to develop theranostic NPs. The artificial chemical-receptor can be expressed on the cell surface in large quantities regardless of the type or subpopulation of the tumor cells. Du et al. prepared a nanoagent (DBCO-ZnPc-LP) by self-assembling a single lipophilic near-infrared (NIR) dye, zinc(II)-phthalocyanine ( $\mathrm{ZnPc}$ ), with a lipid-poly(ethylene glycol) (LP), and then modified it further with dibenzyl cyclootyne (DBCO) to introduce the two-step chemical tumortargeting strategy based on metabolic glycoengineering and click chemistry. ${ }^{228}$ To improve the efficiency of $A c 4 M a n N_{3}$ delivery to the tumor site, the $A c 4 M a n N_{3}$ was encapsulated by DSPS-PEG2000- $\mathrm{NH}_{2}$ to obtain the nanomicelle $\mathrm{Ac}_{4} \mathrm{ManN}_{3}$-LP, which successfully generated artificial receptors after intravenous injection both in vitro and in vivo. DBCO-ZnPc-LP is a multifunctional phototheranostic NP that combines photothermal therapy (PTT) and photoacoustic therapy (PAT). After intravenous injection of DBCO-ZnPc-LP into A549 tumorbearing mice pretreated with either saline or $\mathrm{Ac}_{4} \mathrm{ManN}_{3}$-LP, the PA signal in the tumor sites was substantially higher in the group pretreated with $\mathrm{Ac}_{4} \mathrm{ManN}_{3}$-LP; this may be attributed to the dual targeting effect of EPR and the subsequent binding reaction with the "receptor-like" azides on the tumors. With the significant tumor-targeting strategy, PA imaging, and the synergistic effect of PTT and PAT, the positions of the solid tumors are identified precisely and eradicated completely with few side effects in vivo, compared with PAT or PTT approaches. These targeting NPs can not only convert NIR light into heat for effective thermal ablation but also induce a thermal-enhanced ultrasound shockwave boost to trigger highly localized mechanical damage, achieving a synergistic anti-tumor effect.

Lee et al. also developed NPs based on metabolic glycoengineering and click chemistry associating NIRF and PDT approaches (Figure ). ${ }^{229}$ Like Du et al., they intravenously injected the $\mathrm{Ac}_{4} \mathrm{ManN}_{3}$ after loading it into CNPs to generate artificial receptors. They then developed a second BCN-Ce6-CNP functionalized on the surface by 39 molecules of Chlorin e6 (photosensitizer) and 37 molecules of BCN (bioorthogonal agent), which were intravenously injected into tumor-bearing mice pretreated with saline, free $A_{4} \mathrm{ManN}_{3}$, or $\mathrm{Ac}_{4} \mathrm{ManN}_{3}$-CNP. Signal intensity in the tumor tissue was approximately 1.8 times higher in the mice pretreated with $\mathrm{Ac}_{4} \mathrm{ManN}_{3}$-CNP than in the groups pretreated with saline or free Ac4ManNAz, and more than 10 times higher than in those pretreated with free Ce6. After laser irradiation, significant bleeding and black scab generation were observed in the mice treated with $\mathrm{Ac}_{4} \mathrm{ManN}_{3}$-CNP and $\mathrm{BCN}-\mathrm{Ce} 6-\mathrm{CNP}$. This is direct proof of tumor tissue destruction by excessive local generation of cytotoxic singlet oxygen. 

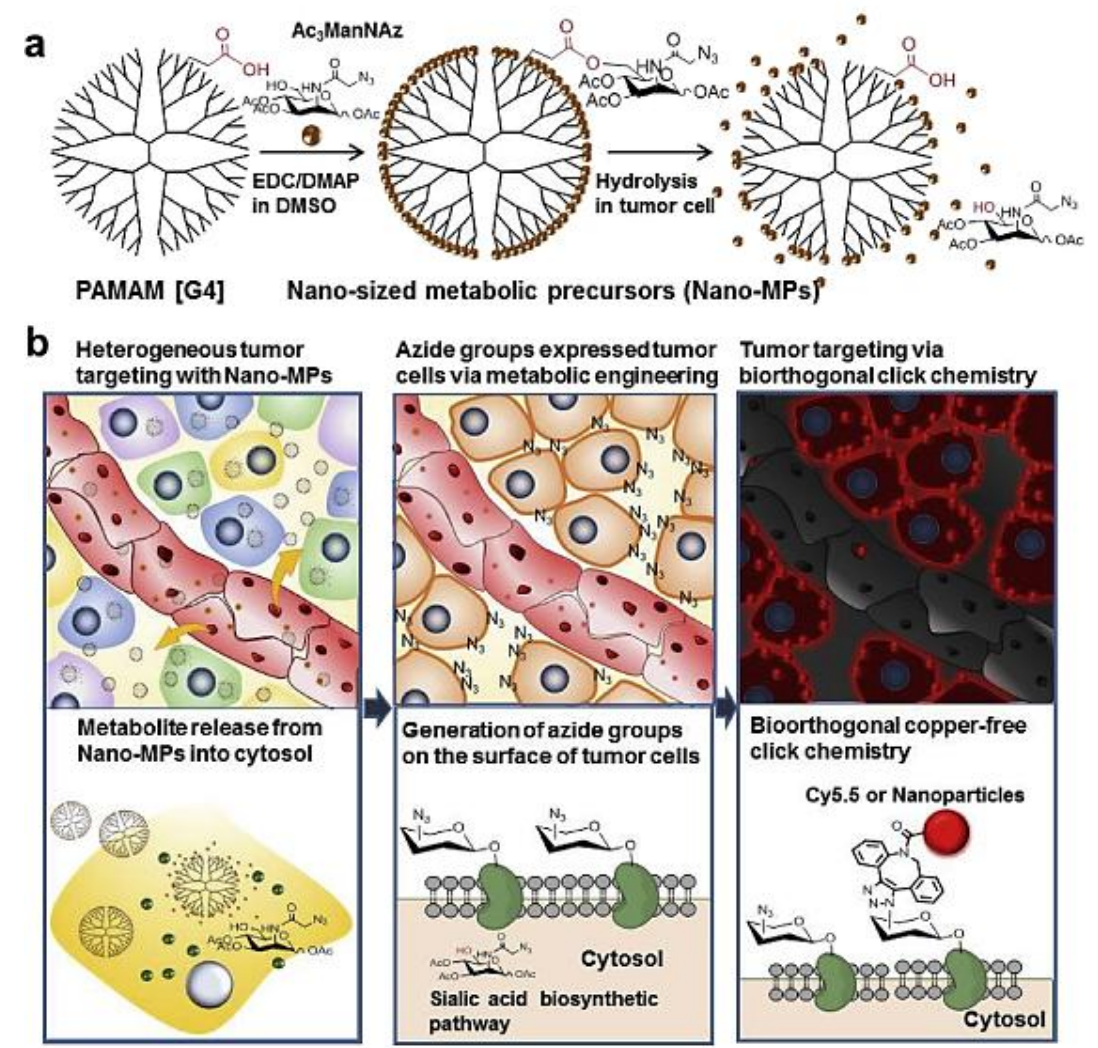

Figure 23. Schemes of the tumor-targeting strategy using nano-sized metabolic precursors (Nano-MPs) and bioorthogonal click chemistry. (a) The synthesis of Nano-MPs containing azide groups (-N3). (b) Illustration for the tumor tissue-specific generation of azide groups by Nano-MPs and metabolic glycoengineering. Then, the generated azide groups on tumor cells can be specifically targeted with bioorthogonal chemical group-conjugated Cy5.5 or liposomes via in vivo bioorthogonal click chemistry. Reprinted adapted with permission from ref ${ }^{229}$. Copyright 2017 Elsevier.

Lu et al. also used the glycoengineering system with IEDDA biorthogonal chemistry to create tetrazine artificial receptors on tumors after intravenous injection of pHLIP-Tz (Figure). ${ }^{208}$ Indocyanine green (ICG)-loaded and trans-cyclooctene (TCO)-conjugated human serum albumin (HSA) nanoparticles (TCO-HSA-ICG NPs, denoted THI) were fabricated as representative carriers and administrated in a second time for both NIRF imaging and PTT. Tumor accumulation in the pTz/THI group was about 2.6 times higher than that of the TH groups. The tumors in the $\mathrm{pTz} / \mathrm{THI}$ group were completely ablated after one session photothermal therapy, with negligible tumor growth or regrowth and a survival rate of $100 \%$ at day 50 . 


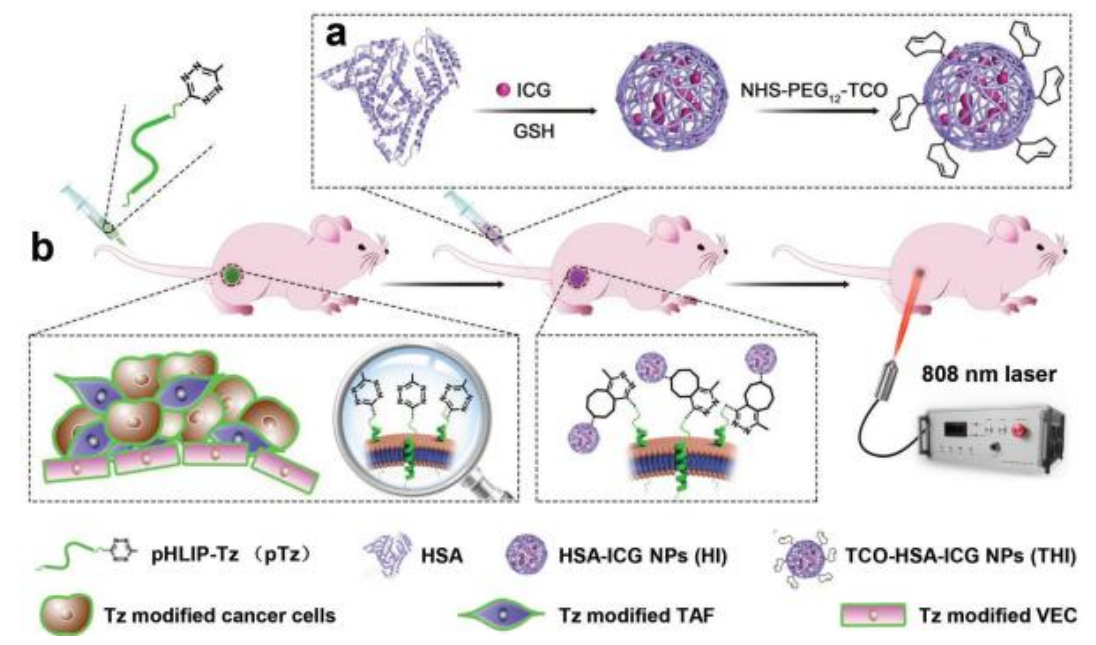

Figure 24. Schematic illustration of the iEDDA-based two-step tumor-targeting strategy. a) Preparation of TCO-HSA-ICG NPS (THI). b) Tz-modified pHLIP (pTz) transferred to tumor in a pH-dependent manner and generated chemical receptors on the membrane of various cells, which were subsequently used as the baits for $\mathrm{THI}$ binding followed with photothermal therapy. Reprinted adapted with permission from ref ${ }^{208}$. Copyright 2018 Wiley-VCH.

Qiao and co-workers developed a copper-free click-targeting LMWH-quercetin nanocomposite system (DLQ/DZ) that improved the specific co-delivery of doxorubicin and the photosensitizer zinc phthalocyanine (PTT application) against breast cancer cells by glycoengineering the tumor cell surface with an azide-modified sugar $\left(A c 4 M a n N_{3}\right)$ (Figure 25). ${ }^{223}$ This co-treatment combination (chemotherapeutic agent and PTT) was studied because it is known that PTT enhances tumor sensitivity to chemotherapeutic agents. The use of artificial receptors and the glycoengineering system necessitated this synergic treatment (with intratumoral rather than intravenous injection of $A c 4 M a n N_{3}$ ). With the DLQ/DZ system, the esterase or acidic tumor environment hydrolyzed the ester bond of the $\mathrm{DLQ}$ to release both $\mathrm{ZnPc}$ and $\mathrm{DOX}$. $\mathrm{ZnPc}$ was then irradiated by NIR laser to increase temperature inside the tumor cells to cause irreversible apoptosis via tumor cell protein denaturation and coagulative necrosis. The authors observed that Ac4ManNAz+DLQ/DZ treatment augmented the anti-cancer effect of combined chemotherapy and PTT $(96.1 \%$ inhibition rate), nearly eliminating the tumor, thus demonstrating the mutually synergistic performance of this therapeutic approach. 


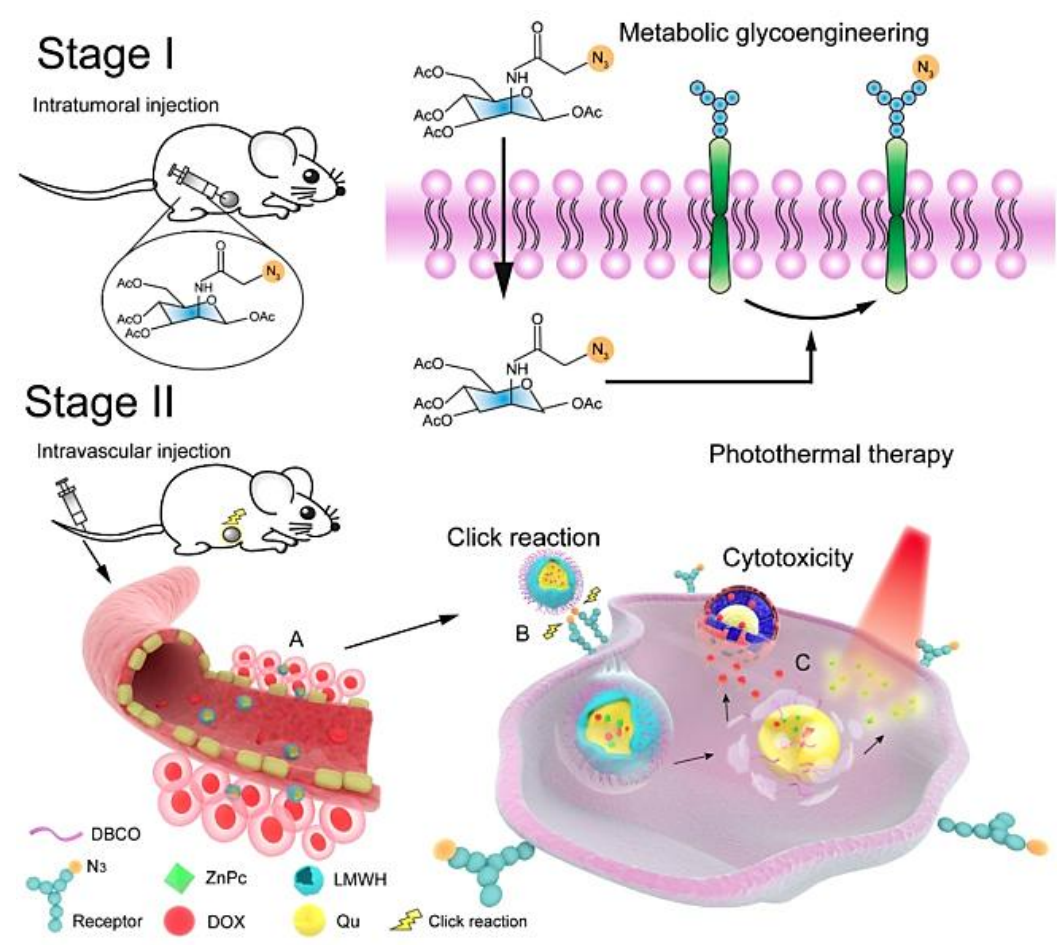

Figure 25. Schematic engineered of the click-targeting nanocomposites for chemo-photothermal synergistic therapy. Stage I: Production of azide groups on the surface of tumor cells by metabolism glycoengineering. Stage II: (A) Nanocomposites accumulated in tumor tissue through EPR effect. (B) Nanocomposites bound to tumor cell via bio-orthogonal copper-free click chemistry. (C) Chemotherapy combined with photothermal therapy to synergize anti-tumor by DNA damage of doxorubicin (DOX) and thermal ablation of zinc phthalocyanine (ZnPc) upon laser irradiation. Reprinted adapted with permission from ref ${ }^{223}$. Copyright 2020 Wiley-VCH.

Xie et al. conducted two very interesting studies on prodrugs that can be cleaved in vivo by a copper-free biorthogonal approach (Figure). ${ }^{230}$ First, they studied a nanosystem for NIRF and prodrug activation. Then, they developed a tetrazine derivative linked with a nearinfrared dye (Tz-NR) to be used as the trigger, as well as a fluorescence probe and the camptothecin vinyl ether prodrug (vinyl ether-masked CPT). The trigger, Tz-NR, mediates cleavage of the vinyl ether group and induces the release of CPT. The molecular prodrug and Tz-NR can be encapsulated separately into liposomes, forming a two-component liposomal bioorthogonal system for imaging and tumor inhibition. As long as the tetrazine is connected to the NR probe, there is no fluorescence (TBET-based system). Fluorescence imaging is only visible once the iEDDA chemical reaction has taken place because the TBET phenomenon is then no longer effective. The presence of fluorescence proves that the CPT has been released. In vivo fluorescence imaging was performed by intravenously injecting both the liposomal NIR fluorescent dye LIP-NR-Tz and the LIP-prodrug into tumor-bearing mice and observing fluorescence images at predetermined times $(0,1,4,12,24$, and 48 hours) after injection. In the tumor region, fluorescence emerged 4 hours after injection, reaching a maximum after 12 hours and then gradually decreasing. The tumor inhibition capability of the liposomal system was investigated in an HeLa xenograft tumor model and proved very effective. High accumulation and retention, associated with the efficiency of the bioorthogonal reaction of the liposomal bioorthogonal system, may contribute to this high anti-tumor efficacy. Based on this success, the authors then developed a similar tetrazinemediated bioorthogonal system for pro-drug activation, photothermal therapy and optoacoustic imaging. This time, the system's trigger was fabricated by immobilizing PEGylated tetrazine on the gold nanorods (AuNR-PEG-Tz), and the bioorthogonal prodrug 
was synthesized by caging the drug camptothecin with vinyl ether, and encapsulating it with phospholipid liposomes (LIP-VE-CPT). The combined therapy group (AuNR-PEG-Tz + LIPVE-CPT + laser) displayed the highest tumor inhibition efficacy and the lowest relative tumor volume during this investigation.

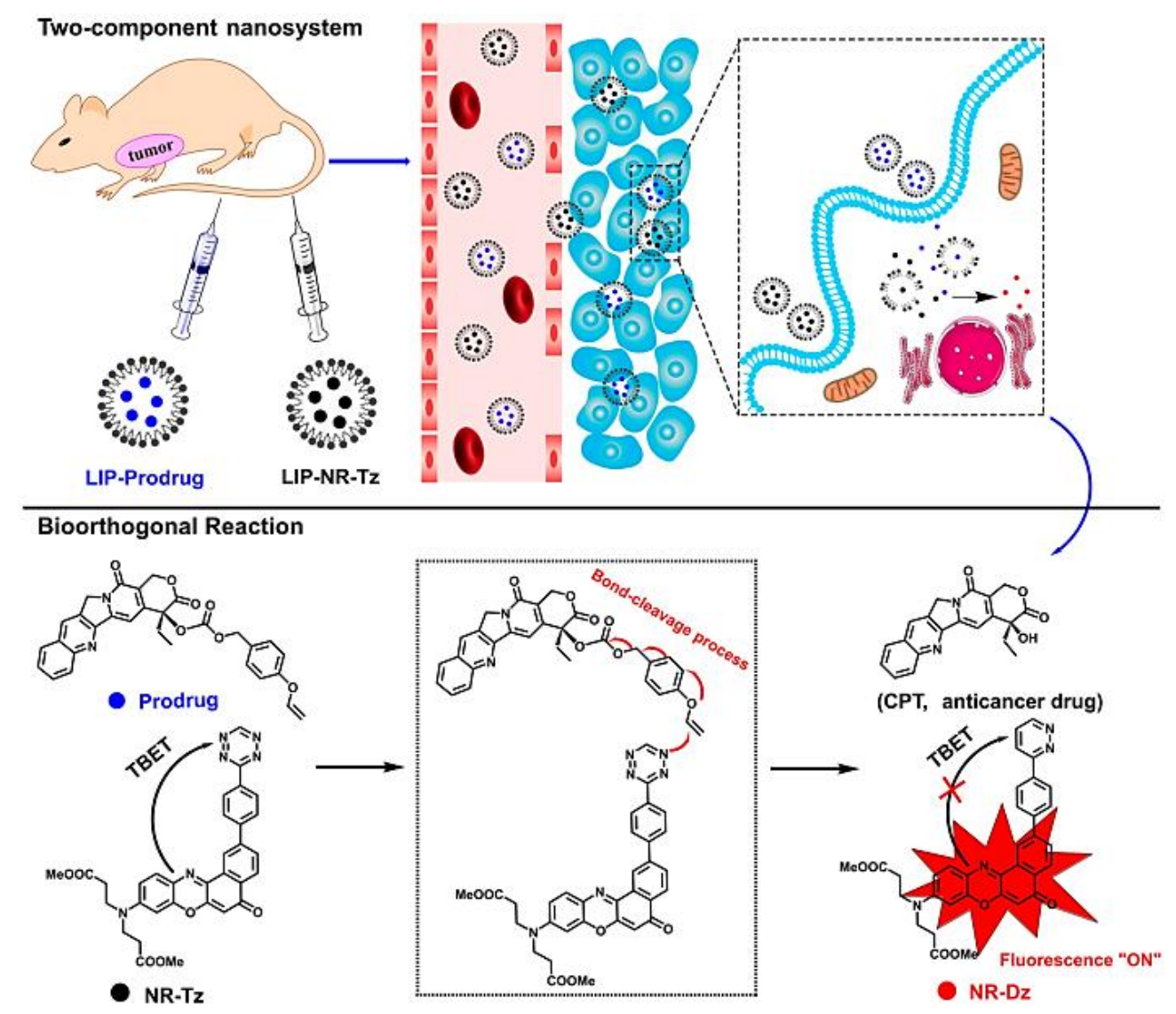

Figure 26. Schematic overview of the two-component Bioorthogonal Nanosystem for Imaging and Tumor inhibition. Reprinted adapted with permission from ref ${ }^{230}$. Copyright 2019 American Chemical Society.

\subsection{NPs using click chemistry for imaging modality and a theranostic approach}

Wang et al. developed a dual-labeled iRGD-modified multifunctional porous silicon nanoparticle (Psi NP) modified by DBCO and amino groups on its surface and able to encapsulate a chemotherapeutic agent (sorafenib). ${ }^{134}$ The Alexa Fluor 488 and DBCO were conjugated with the Psi NPs via amide linkage. The DBCO groups were then used to graft both peptide iRGD and macrocycle DOTA for radiolabeling via SPAAC. Mice bearing prostate cancer xenografts were studied by SPECT imaging, and in vivo biodistribution of ${ }^{111}$ In-radiolabeled multifunctional Psi and Psi-iRGD NPs was determined after intravenous administration. The intravenously administered iRGD-modified NPs achieved higher tumorspecific accumulation compared with the PSi NPs, based on the 4.4 vs. 2.7 tumor-to-muscle ratios of the injected dose per gram (ID\%/g), for Psi-iRGD and Psi, respectively. iRGD peptide surface modification of the nanocarriers increased the tumor accumulation of the latter. The antitumor effect of the multifunctional NPs loaded with Sorafenib (SF) was then assessed in vivo. After two cycles of intravenous administration 24 hours apart, both SFloaded NPs (Psi-SF and PSi-iRGD-SF corresponding to $3 \mathrm{mg} / \mathrm{kg}$ of SF) affected tumor growth in a manner similar to that of the free SF. The authors indicated that this 
disappointing result was probably due to the fast release of the SF from the Psi matrix into the blood after intravenous administration. Nevertheless, the authors demonstrated the development and efficacy of a multifunctional Psi nanocarrier, making the multimodal nanosystem presented a promising nanotheranostic PSi system for cancer diagnosis and treatment.

\section{Nanoparticles, click chemistry and protein corona}

Through the different examples from the literature cited in the previous paragraphs of this review, we can ascertain the rightful place of click chemistry in the field of nanomedicine. Although the literature proposes few comparisons of traditional functionalization of NPs and click chemistry, it seems very clear that this chemistry constitutes an asset for grafting molecules, radiosensitizing elements, optical imaging or ligands, regardless of the type of NP. Compared with traditional coupling reactions, which require organic solvents, high temperature/pressure or toxic catalysts, click chemistry avoids these constraints and represents an opportunity for an effective, direct reaction in the aqueous phase in biological matrices (Table 6).

Table 8. Main advantages and disadvantages of click chemistry reactions currently used in nanomedicine

\begin{tabular}{|c|c|c|}
\hline $\begin{array}{c}\text { Click } \\
\text { chemistry }\end{array}$ & Advantages & Disadvantages \\
\hline CuAAC & 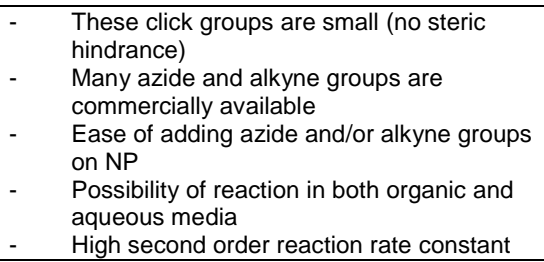 & $\begin{array}{l}\text { Requires copper catalysis and risk of copper } \\
\text { contamination on the surface of NP (problem for } \\
\text { human application) } \\
\text { Risk of interaction between azide groups on the } \\
\text { surface of NP due to the zwitterionic form }\end{array}$ \\
\hline Thiol-ene & $\begin{array}{ll}- & \text { Ease of adding alkene groups on NP } \\
- & \text { Click groups are small (no steric hindrance) } \\
- & \text { Possibility of reaction in both organic and } \\
\text { - } & \text { Squeous media } \\
& \text { Soft conditions ( } \mathrm{pH}, \text { temperature) for the } \\
\text { reaction } & \text { No catalyst }\end{array}$ & $\begin{array}{ll}- & \text { Difficulties working with thiol groups } \\
\text { - } & \text { Risk of polymerization between different alkene } \\
\text { groups on the surface of NP } \\
\text { - } \quad \text { Risk of formation of intra or inter disulfide bridge } \\
\text { Risk of competition with other thiols of the } \\
\text { environment }\end{array}$ \\
\hline SPAAC & $\begin{array}{ll}- & \text { Bioorthogonal entities are commercially } \\
\text { - } & \text { Movailable } \\
\text { - } & \text { Reaction in both organic and aqueous } \\
\text { - } & \text { media } \\
\text { Soft conditions ( } \mathrm{pH}, \text { temperature) for the } \\
\text { reaction } \\
\text { - One small and one large structure } \\
\text { No catalyst }\end{array}$ & $\begin{array}{ll}\text { - } & \text { Risk of p-stacking with strained-alkyne group if } \\
\text { their density is too high on the surface of NP } \\
\text { - } \quad \text { Moderate second order reaction rate constant }\end{array}$ \\
\hline IEDDA & $\begin{array}{ll}- & \text { Biorthogonal entities are commercially } \\
\text { available } \\
\text { - }\end{array}$ & $\begin{array}{ll}- & \text { Isomerization of TCO } \\
- & \text { Risk of p-stacking with tetrazine groups if their } \\
\text { density is too high on the surface of NP } \\
\text { Instability of the reactive groups under too acidic or } \\
\text { basic conditions } \\
\text { Two large structures }\end{array}$ \\
\hline
\end{tabular}

The thiol-ene approach is much less used than the other three click reactions. However, it could prove to be relevant with regard to the biomolecules that can be grafted onto the NPs. No azide or alkyne functional groups are found among native biomolecules, thus imposing the specific introduction of these groups into proteins or DNA. Compared with the azide- 
alkyne reaction, the thiol functional group of cysteine-containing proteins makes bioconjugation more readily achievable through a thiol-ene click reaction. ${ }^{62,232}$

One of the main advantages of click chemistry is that it appears to allow the modification of molecules or ligands on the surface of NPs, regardless of the design of the nano-object itself. Once the click entities are available on the surface of the nanoparticle, they can be used to graft different groups depending on the desired application. This is particularly important, since many molecules functionalized with bioorthogonal entities are now commercially available. The bioorthogonality of these different reactions thus makes it possible to envisage incorporating different functionalities into the same NP (for example, a fluorescent probe, a drug, a peptide, a monoclonal antibody, etc.). Concerning the PT approach, we think that a single nano-object, functionalized on the surface with bioorthogonal entities, could be used for different cancer pathologies. The complementary bioorthogonal entities simply have to be grafted onto monoclonal antibodies with a high affinity for the receptors (over)expressed in the tumor. However, click chemistry is not yet a paradigm in nanomedicine. When NPs are exposed to biological fluids and/or a tumoral microenvironment, their surface becomes covered with biomolecules and/or proteins. The "protein corona" that forms around the NPs is described as "hard" or "soft", depending on the binding of the proteins adsorbed onto the surfaces of the NPs. The energetically favorable process of protein corona formation is very complex and has a significant impact on the original physicochemical properties of the NPs, possibly altering their biological identity between in vitro and in vivo evaluations. ${ }^{233}$ The presence of proteins is all the more important in the PT strategy. Aside from the possible steric hindrance of the covalent reaction between the two-bioorthogonal entities present respectively on the antibody and the NP, the PT strategy cannot be implemented if the protein corona prevents the click reaction at the tumor site. Moreover, in the case of the PT strategy, can we still use the term active targeting? For nanoparticles, the notion of anchoring is likely to be more relevant than the concept of active targeting. For the PT strategy, the NP, which is injected in a second step, is distributed in vivo by the EPR effect, and then "attached" to the mAb when click chemistry takes place at the tumor site.

In any case, perfect engineering of the surface ligands is essential for the future of PT approaches with NPs. Recent works have discussed the importance of using non-fouling moieties to prevent protein corona formation as this protein layer affects the properties of nanomaterials, altering their behavior and masking engineered functionalities. ${ }^{234}$ Jiang et al. recently published a complete review of antifouling strategies for selective in vitro and in vivo sensing. ${ }^{235} \mathrm{~A}$ wide range of molecular systems, such as polyethyleneglycol (linear or branched PEG, >10 EG units), zwitterionic species, peptides with alternating or random mixed-charge and other polymers like polysaccharides, polyoxazolines, poly(hydroxy acrylates), and hyperbranched polyglycerol (HPG), have been shown to possess significant antifouling properties, mainly resulting from their high hydration. Moyano et al. first described the antifouling effect of sulfobetaine zwiterrionic head groups for NPs. AuNPs coated with PEG3-sulfobeteaine ligands did not adsorb proteins at moderate serum protein concentrations ( 10 to $55 \%$ plasma). ${ }^{236}$ Another team found that sulfobetaine arms provided a corona-free property, even in the presence of other functions. ${ }^{237}$ The dual functionalization of NPs is a promising strategy for targeted delivery purposes, because careful balance of a zwitterionic moiety and a proper targeting function on the NP surface should control the bionano interaction while maintaining targeting capabilities. However, the choice of the best surface ligands will depend on the in vivo route chosen and the therapeutic strategy. The 
dilemma might be the choice of an appropriate NP design. Surface engineering will differ depending on whether or not we want to activate the cell's uptake machinery in a process known as endocytosis. Zhang and his colleagues referred to a soft reversible adsorption of the protein corona on $2 \mathrm{~nm}$ core AuNPs coated with a PEG3-quaternary ammonium ligand. ${ }^{238}$ In this study, no adsorption was observed with the PEG3-sulfobetaine ligand. Ultimately, the antifouling effect does not allow cell uptake of NPs by endocytosis. In this case, the best functionalization strategy involved the PEG3-ammonium ligand, allowing soft and reversible adsorption of the proteins associated with cell penetrating by endocytosis. This functionalization method will probably not be preferred for in vivo pre-targeting. Very densely charged surfaces with zwitterionic ligands will be preferred to avoid any protein adsorption and thus allow the in vivo click reaction. It is therefore important to conduct studies on the formation of this protein corona depending on the nature of the bioorthogonal entities grafted onto the surface of the NPs, their number, and the overall charge of the nano-object before assuming that the PT approach can be applied to any type of nanoparticle.

\section{CONCLUSIONS AND OUTLOOK}

Theranostic NPs have the potential to revolutionize future disease management. In particular, their development has emerged as an encouraging strategy for personalized medicine in the fields of cancer diagnosis and therapy. However, their decoration still encounters various engineering difficulties, including achieving good reproducibility of nanoparticle batches, making them biocompatible, and improving their ability to target the TME specifically. Even a decade ago, most NPs were surface functionalized by carbodiimide and maleimide reactions.

Advances in organic chemistry have apparently opened up a new field of NP engineering. The click chemistry discovered by Huisgen in the 1960s represents a practical organic reaction for the quantitative synthesis of progressively growing polymers. However, Sharpless coined the term "click chemistry" to define highly efficient synthetic reactions which tolerate various functional groups and occur under mild synthetic conditions. Numerous published studies highlight the advantages of click chemistry as a ligation tool for potential applications as non-targeting nanocarriers or theranostic vehicles for cancers. High yields (often higher than 95\%), specificity, diverse reaction conditions and scale-expansion are assets for click chemistry, offering the possibility of creating complex, stable NPs. This is attracting growing interest for the surface incorporation of non-targeted molecules (e.g. fluorophores and contrast agents for multimodal imaging) and for the specific and efficient encapsulation of insoluble chemotherapeutic agents to improve the therapeutic index. Micelles, liposomes, dendrons, dendrimers and polymeric NPs thus continue to be the nanocarriers most employed in this area. They show efficient cell internalization and antiproliferative activity, even if, for most of them, efficacy remains to be evaluated either in vivo or in clinics.

Click chemistry also allows the grafting of ligands onto NPs for active targeting and accumulation in specific cancer cells. Regarding the comparison of clicked targeted NPs and non-targeted NPs, active targeting seems to be especially effective thanks to the favorable and selective interaction of NPs with their biological targets. Targeting ligands allow restricted access only to cancer cells for the intracellular delivery of genes (i.e., for gene knockdown), therapeutic agents (i.e., for an anticancer effect) or imaging agents. They substantially improve cellular uptake and internalization, generally allowing the promotion of 
cancer cell cytotoxicity, apoptosis and transfection efficiency through the well-exploited receptor-mediated endocytosis pathway. However, CuAAC is a cooper-catalyst reaction and, despite engineering developments to purify these nanosystems, residual traces of copper may be present on the nano-object, thus posing a problem for clinical transfer.

The concept of bioorthogonal chemistry (copper-free click chemistry) seems capable of solving this bioaccounting problem and suggests the advent of a new era for therapeutic and theranostic NPs. The notion of bioorthogonality was developed by Bertozzi to define a chemistry that required that the functional groups be inert towards biological macromolecules. Bioorthogonality ensures a reaction capable of taking place under physiological conditions ( $\mathrm{pH}$ and temperature) while avoiding toxicity in living systems. SPAAC and IEDDA systems certainly seem to characterize the future of nanomedicine for several reasons:

(1) These two systems appear to have constituted a "classical" functionalization chemistry in the field of NPs

(2) No catalyst is needed to conjugate the two-bioorthogonal entities and they are applicable regardless of the type of NP: organic or inorganic.

The PT approach appears very promising for nanomedicine. According to this strategy, the NPs injected in a second step distribute themselves due to the EPR effect. It is also true that, according to Wilhem's meta-analysis, less than $1 \%$ of the injected NPs reach the tumor volume. However, a first injection of the modified antibody appears to allow better tumor concentration of the NPs by click reaction, as demonstrated by Haun et al., who compared BOND-1 (one step targeting) and BOND-2 (two-step targeting) approaches. ${ }^{202}$

As the antibody is dissociated from the NP, several perspectives can be envisaged:

- This approach would make it possible to envisage using the same NPs for the same type of cancer but with different mAbs targeting different biomarkers of interest. For example, a single NP could be used with either anti-EGFR or anti-HER2 mAb for breast cancer. If this in vivo ligation is effective with an anticancer mAb, we can assume that it would also be effective with mAbs used for other pathologies, such as inflammation for example.

However, some significant questions and problems still remain to be resolved:

The PT approach is only possible if the antibodies modified with the first bioorthogonal entity do not internalize before administration of the NPs. But do the bioorthogonal entities promote internalization of the modified antibody? If this difficulty is confirmed, it will probably be necessary to develop engineered antibodies to limit their internalization over the first 24 or 48 hours in order to allow grafting of the NP onto the tumor. Will different approaches be required for chimeric, humanized and human antibodies?

As the mAb is not directly grafted onto the NP, the size and surface of the NP must be controlled to avoid both renal clearance and uptake by the mononuclear phagocyte system after formation of a protein corona that contains ions, opsonins and other serum proteins, thus maximizing blood circulation time and allowing the NPs to reach the tumor site to enable the click reaction. 
The NPs must be functionalized on the surface to avoid the formation of the protein corona which would be partly responsible for non-ligation in vivo.

Rather than using antibodies, should we not develop the notion of glycoengineering to modify the surface of the tumors and allow the attachment of NPs in a second phase?

Regardless of the approach used (passive or active targeting in two stages (PT approach or glycoengineering), it is clear that biodistribution of the NP will take place due to the EPR effect. One solution for developing multimodal or theranostic NPs may therefore be an approach where the NP acts as a "tumor platform", with bioorthogonal entities on the surface capable of grafting theranostic agents, PTT or radiosensitive agents, or even prodrugs in a second phase.

\section{AUTHOR INFORMATIONS}

\section{Corresponding Authors}

Emmanuel Moreau - Université Clermont Auvergne, Imagerie Moléculaire et Stratégies Théranostiques, BP 184, F-63005 Clermont-Ferrand, France. Inserm, U 1240, F-63000 Clermont-Ferrand, France. Centre Jean Perrin, F-63011 Clermont-Ferrand, France.; orcid.org/0000-0002-8004-5019; Email: emmanuel.moreau@uca.fr.

Carole Chaix - Interfaces and Biosensors, UMR 5280, CNRS, Villeurbanne, France; orcid.org/0000-0003-4882-2364; Email : carole.chaix-bauvais@univ-lyon1.fr.

\section{Authors}

Ludivine Taiariol - Université Clermont Auvergne, Imagerie Moléculaire et Stratégies Théranostiques, BP 184, F-63005 Clermont-Ferrand, France. Inserm, U 1240, F-63000 Clermont-Ferrand, France. Centre Jean Perrin, F-63011 Clermont-Ferrand, France.; 00000003-4666-0410 ; Email : taiariol@outlook.com.

Carole Farre - Université de Lyon, CNRS, Université Claude Bernard Lyon 1, Institut des Sciences Analytiques, UMR 5280, 5 rue de la Doua, F-69100 Villeurbanne, France; orcid.org/0000-0003-0783-289X; Email : carole.farre@univ-lyon1.fr.

\section{Author Contributions}

The manuscript was written through contributions of all authors. All authors have given approval to the final version of the manuscript.

\section{Notes}

The authors declare no competing financial interest 


\section{Biographies}

Ludivine Taiariol obtained her MSc in Drug Sciences from the University of ClermontFerrand (France) in 2016. She then joined UMR 1240 INSERM/IMoST research laboratory for her Ph.D. under the supervision of Doctor Emmanuel Moreau and Doctor Carole Chaix. She received her Ph.D. in Chemistry of Materials, Nanomaterials and Processes from the University of Clermont-Ferrand (France) in 2019. Her thesis subject focused on the development of novel silica nanoparticles for the application in external radiotherapy. Her skills are at the interface between organic chemistry/chemistry of nanomaterials and biochemistry.

Carole Chaix received her Ph.D. in Biological and Medical Engineering from the University of Grenoble (France) in 1990. She is presently Research Director at the Institute of Analytical Sciences of Lyon and head of the Interfaces and Biosensors group. She has extensive experience in the functionalization of biomolecules and the surface chemistry of materials. Her research focus on the development of bio-functionalized nanomaterials and bioanalytical systems such as biosensors or biochips for applications in nanomedicine and biological analysis. She is the author or co-author of more than 80 articles and book chapters.

Carole Farre obtained her MSc in Biomolecular Chemistry from the University of Montpellier (France) in 2006. She is currently working as an Engineer at the Institute of Analytical Sciences of Lyon. Her research focuses on nanoparticle functionalization with biomolecules, in particular DNA. She is also working on new molecules for DNA labelling and their integration into bioanalytical systems.

Emmanuel Moreau received his Ph.D. in pharmaceutical sciences from the University of Clermont-Ferrand (France) in 2001. He did post-doctoral studies at the Saint-François d'Assises research centre in Quebec City (Canada) from 2002 to 2004 . He is presently assistant professor at the Faculty of Pharmacy in Clermont-Ferrand (France). Since 2005, he has been conducting his research in an INSERM unit in Clermont-Ferrand (France). He is the leader of the "Targets and tools for imaging and therapy" team combining the complementary expertise of chemists, radiochemists, biologists and clinicians. This research focuses on the development of new radiopharmaceuticals for melanoma, colorectal cancer and breast cancer. He is author and co-author of about fifty publications and has supervised about ten Ph.D students.

\section{ACKNOWLEDGEMENTS}

This project is based upon work supported in part by the "Cancéropôle Rhône-Alpes Auvergne CLARA Oncostarter" (Grant support CVPPRCAN000184) (E.M), the "Ligue Régionale contre le Cancer" (Grant support R21JPRARPDD) (E.M), Ludivine Tairaiol's doctoral work was supported by funding from the French Ministry of Research and the doctoral school of basic sciences in Clermont-Ferrand (2016-2019).

\section{ABBREVIATIONS}

$\mathrm{Ac}_{4} \mathrm{MAnNAz}=$ tetraacetylated $\mathrm{N}$-azidoacetyl-D-mannosamine

AMP $=$ activatable MMP-specific peptide probe

AuNP = gold nanoparticle 
AuNC $=$ gold nanocluster

AuNR: gold nanorod

$\mathrm{AZT}=$ acetazolamide

$\mathrm{BBB}=$ Blood-brain barrier

$\mathrm{BCN}=$ bicyclo[6.1.0]nonyne

BHQ-3 = non-fluorescent Black Hole Quencher-3

BOND = bioorthogonal NP detection

$\mathrm{CA}=$ carbonic anhydrase

CD20: $\beta$-lymphocyte antigen

Ce6 $=$ Chlorin e6

CF7N = Cy7-modified chitosan nanoparticle

$\mathrm{CPP}=$ cell penetrating peptide

$\mathrm{CPT}=$ camptothecin

$\mathrm{CT}=$ computed tomography

CuAAC $=$ copper $(\mathrm{I})$-catalyzed Azide-Alkyne [3+2] Cycloaddition

$\mathrm{CDDP}=$ cisplatin

$\mathrm{CNP}=$ chitosan nanoparticle

$\mathrm{CPP}=$ cell penetrating peptide

$\mathrm{CPT}=$ camptothecin

CSPT $=$ chain-shattering polymeric therapeutics

Cy = cyanine

$\beta-C D=\beta$-cyclodextrin

$\mathrm{DACH}-\mathrm{Pt}=$ dichloro(1,2-diaminocyclohexane)platinum(II)

$\mathrm{DBCO}=$ dibenzocyclooctyne

$D L Q=D B C O-L Q$

D-mino = hydroxyl-G6 PAMAM dendrimer-9-amino-minocycline conjugate

DOTA $=1,4,7,10$-Tetraazacyclododecane-1,4,7,10-tetraacetic acid

DOX $=$ doxorubicin

DSPS $=1,2$-Distearoyl-sn-glycero-3-phospho-L-serine

$D Z=D O X-z i n c$ phthalocyanine conjugate

EDC = 1-ethyl-3-(3-dimethylaminopropyl)carbodiimide

EGFR $=$ epidermal growth factor receptor 
$\mathrm{EPR}=$ enhanced permeability and retention

$F A=$ folic acid

$\mathrm{FCPZnO}=\mathrm{FA}$-conjugated hollow $\mathrm{ZnO}$ nanoparticle

FITC= fluorescein isothiocyanate

$\mathrm{FNC}=$ folate-decorated nanoceria

FND = fluorescent nanodiamond

$F R=$ folate receptor

GEM = gemcitabine

GFP $=$ green fluorescent protein

$\mathrm{GLA}=$ glutamate urea

GON = gelatin-oleic acid nanoparticle

$\mathrm{GSH}=$ glutathione

GT = ganetespib

$\mathrm{HA}=$ hyaluronic acid

HAase = hyaluronidase

HDAC = protein histone deacetylase

HDACi = histone deacetylase inhibitor

HER2 = human epidermal growth factor receptor 2

$\mathrm{HNE}=$ epithelial tumor cell line

ICP = infinite-coordination-polymer

IC50 $=$ half-maximal inhibitory concentration

IEDDA = inverse-electron-demand Diels-Alder [4+2]

LIP = liposome

$\mathrm{LHRH}=$ luteinizing hormone releasing hormone

$\mathrm{LMWH}=$ low molecular weight heparin

$L Q=(L M W H)-q u e r c e t i n(Q u)$ conjugate

$\mathrm{mAb}=$ monoclonal antibody

$\mathrm{MBG}=$ mesoporous bioactive glass

MCF-7 = human breast adenocarcinoma cell line

MDA-MB = epithelial, human breast cancer cell line

$\mathrm{MFI}=$ mean fluorescence intensity

MFNP = magneto-fluorescent nanoparticle 
MMP = matrix metalloproteinase

MPLA = monophosphoryl lipid A

$\mathrm{MR}=$ magnetic resonance

MRI: magnetic resonance imaging

MRSA = methicillin-resistant Staphylococcus aerus

MSN = mesoporous silica nanoparticle

MTX = methotrexate

$\mathrm{NC}=$ nanoconjugate

$\mathrm{ND}=$ nanodiamond

$\mathrm{NHS}=\mathrm{N}$-hydroxysuccinimide

$\mathrm{NIR}=$ near infrared

NIRF: near infrared fluorescence

NMOFs $=$ metal-organic framework nanoparticles

$\mathrm{NP}=$ nanoparticle

PAMAM = polyamidoamine

$\mathrm{PAT}=$ photoacoustic therapy

$\mathrm{PDA}=$ polydopamine

PD-L1 = programmed death-ligand 1

PDT = photodynamic therapy

PEG = polyethylene glycol

$\mathrm{PET}=$ positron-emission tomography

$\mathrm{pHLIP}=\mathrm{pH}(\mathrm{low})$ insertion peptide

pHLIP-Tz $=\mathrm{pH}$ (low) insertion peptide conjugated to tetrazine

PLGA = poly(lactic-co-glycolic acid $)$

$\mathrm{PT}=$ pretargeting

$\mathrm{PTT}=$ photothermal therapy

PTX = paclitaxel

PSMA = prostate-specific membrane antigen

Psi $=$ porous silicon

$\mathrm{QD}=$ quantum dot

$\mathrm{RAW}=$ monocyte/macrophage-like cell line

RGD = Arginine-Glycine-Aspartic acid 
RGE = neuropilin-1-targeted peptide (RGERPPR)

$\mathrm{RTX}=$ rituximab

$\mathrm{ROMP}=$ ring-opening metathesis copolymerization

$\mathrm{RSC}=$ lipid-based complexes encapsulating siRNA

$\mathrm{RTV}=$ relative tumor volume

SERS = Surface-Enhanced Raman Scattering

siRNA = small interfering RNA

SKOV-3 = ovarian cancer cell line

SMNPs = small molecule-based nanoparticles

SN-38 = 7-ethyl-10-hydroxycamptothecin

SPAAC $=$ Strain-Promoted Alkyne-Azide Cycloaddition

SPECT $=$ single-photon emission computed tomography

SPION = SuperParamagnetic Iron Oxide nanoparticle

$\mathrm{SPR}=$ surface plasmon resonance

$\mathrm{TAF}=$ tumor-associated fibroblasts

Tat $=$ Trans-activating transcriptional activator

TBET $=$ through-bond energy transfer

$\mathrm{TCO}=$ trans-cyclooctene

$\mathrm{TME}=$ tumor microenvironment

TNF- $\alpha=$ tumor necrosis factor $\alpha$

TPETM

2-(1-(5-(4-(1,2,2-tris(4-methoxyphenyl)vinyl)phenyl)thiophen-2-yl)ethylidene)malononit rile

$\mathrm{Tz}=$ tetrazine

UCNP: upconversion nanoparticle

USPION = Ultra Small SuperParamagnetic Iron Oxide nanoparticle

VEC $=$ vascular endothelial cells

$\mathrm{ZnPc}=$ zinc phthalocyanine

\section{REFERENCES}

(1) Dr. Niranjani Chaurasia. Nanotechnology and Nanomaterials in Everyday Life. Int. J. Sci. Res. IJSR 2017, 6, 1560-1562. 
(2) Weltring, K.-M.; Gouze, N.; Martin, N.; Pereira, N.; Baanante, I.; Gramatica, F. Nanomedicine Strategic Research and Innovation Agenda. Nanomedicine European Technology Platform January 2016.

(3) Nakhlband, A.; Eskandani, M.; Omidi, Y.; Saeedi, N.; Ghaffari, S.; Barar, J.; Garjani, A. Combating Atherosclerosis with Targeted Nanomedicines: Recent Advances and Future Prospective. Biolmpacts 2018, 8, 59-75.

(4) Jiang, W.; Rutherford, D.; Vuong, T.; Liu, H. Nanomaterials for Treating Cardiovascular Diseases: A Review. Bioact. Mater. 2017, 2, 185-198.

(5) Kanwar, J. R.; Sun, X.; Punj, V.; Sriramoju, B.; Mohan, R. R.; Zhou, S.-F.; Chauhan, A.; Kanwar, R. K. Nanoparticles in the Treatment and Diagnosis of Neurological Disorders: Untamed Dragon with Fire Power to Heal. Nanomedicine Nanotechnol. Biol. Med. 2012, 8, 399-414.

(6) Zazo, H.; Colino, C. I.; Lanao, J. M. Current Applications of Nanoparticles in Infectious Diseases. J. Controlled Release 2016, 224, 86-102.

(7) DiSanto, R. M.; Subramanian, V.; Gu, Z. Recent Advances in Nanotechnology for Diabetes Treatment: Nanotechnology for Diabetes Treatment. Wiley Interdiscip. Rev. Nanomed. Nanobiotechnol. 2015, 7, 548-564.

(8) lavicoli, I.; Fontana, L.; Leso, V.; Bergamaschi, A. The Effects of Nanomaterials as Endocrine Disruptors. Int. J. Mol. Sci. 2013, 14, 16732-16801.

(9) Oliveira, I. M.; Gonçalves, C.; Reis, R. L.; Oliveira, J. M. Engineering Nanoparticles for Targeting Rheumatoid Arthritis: Past, Present, and Future Trends. Nano Res. 2018, 11, 4489-4506.

(10) Riffault, M.; Six, J.-L.; Netter, P.; Gillet, P.; Grossin, L. PLGA-Based Nanoparticles: A Safe and Suitable Delivery Platform for Osteoarticular Pathologies. Pharm. Res. 2015, 32, 3886-3898.

(11) Awasthi, R.; Roseblade, A.; Hansbro, P. M.; Rathbone, M. J.; Dua, K.; Bebawy, M. Nanoparticles in Cancer Treatment: Opportunities and Obstacles. Curr. Drug Targets 2018, 19, 1696-1709.

(12) Riehemann, K.; Schneider, S. W.; Luger, T. A.; Godin, B.; Ferrari, M.; Fuchs, H. Nanomedicine-Challenge and Perspectives. Angew. Chem. Int. Ed. 2009, 48, 872897.

(13) Prabhu, P.; Patravale, V. The Upcoming Field of Theranostic Nanomedicine: An Overview. J. Biomed. Nanotechnol. 2012, 8, 859-882.

(14) Baetke, S. C.; Lammers, T.; Kiessling, F. Applications of Nanoparticles for Diagnosis and Therapy of Cancer. Br. J. Radiol. 2015, 88, 20150207.

(15) Greish, K.; Mathur, A.; Bakhiet, M.; Taurin, S. Nanomedicine: Is It Lost in Translation? Ther. Deliv. 2018, 9, 269-285.

(16) Wilhelm, S.; Tavares, A. J.; Dai, Q.; Ohta, S.; Audet, J.; Dvorak, H. F.; Chan, W. C. W. Analysis of Nanoparticle Delivery to Tumours. Nat. Rev. Mater. 2016, 1, 16014.

(17) Meldal, M.; Tornøe, C. W. Cu-Catalyzed Azide-Alkyne Cycloaddition. Chem. Rev. 2008, 108, 2952-3015.

(18) Wang, Q.; Chan, T. R.; Hilgraf, R.; Fokin, V. V.; Sharpless, K. B.; Finn, M. G. Bioconjugation by Copper(I)-Catalyzed Azide-Alkyne [3 + 2] Cycloaddition. J. Am. Chem. Soc. 2003, 125, 3192-3193.

(19) Verma, S. A Novel Loom of Click Chemistry in Drug Discovery. Int. J. Drug Dev. Res. 2015, 18-21.

(20) Bertozzi, C. R. Chemical Glycobiology. Science 2001, 291, 2357-2364.

(21) Kim, E.; Koo, H. Biomedical Applications of Copper-Free Click Chemistry: In vitro, in vivo, and Ex Vivo. Chem. Sci. 2019, 10, 7835-7851.

(22) Ladd, E.; Sheikhi, A.; Li, N.; van de Ven, T. G. M.; Kakkar, A. Design and Synthesis of Dendrimers with Facile Surface Group Functionalization, and an Evaluation of Their Bactericidal Efficacy. Molecules 2017, 22, 868-896.

(23) Anandkumar, D.; Rajakumar, P. Synthesis and Anticancer Activity of Bile Acid Dendrimers with Triazole as Bridging Unit through Click Chemistry. Steroids 2017, 125, 37-46. 
(24) Sadowski, L. P.; Edem, P. E.; Valliant, J. F.; Adronov, A. Synthesis of Polyester Dendritic Scaffolds for Biomedical Applications. Macromol. Biosci. 2016, 16, 14751484.

(25) Fischer, G.; Wängler, B.; Wängler, C. Optimized Solid Phase-Assisted Synthesis of Dendrons Applicable as Scaffolds for Radiolabeled Bioactive Multivalent Compounds Intended for Molecular Imaging. Mol. Basel Switz. 2014, 19, 6952-6974.

(26) Singh, P.; Pandit, S.; Mokkapati, V. R. S. S.; Garg, A.; Ravikumar, V.; Mijakovic, I. Gold Nanoparticles in Diagnostics and Therapeutics for Human Cancer. Int. J. Mol. Sci. 2018, 19, 1979-1995.

(27) Mao, W.; Kim, H. S.; Son, Y. J.; Kim, S. R.; Yoo, H. S. Doxorubicin Encapsulated Clicked Gold Nanoparticle Clusters Exhibiting Tumor-Specific Disassembly for Enhanced Tumor Localization and Computerized Tomographic Imaging. J. Control. Release 2018, 269, 52-62.

(28) Tural, S.; Ece, M. Ş.; Tural, B. Synthesis of Novel Magnetic Nano-Sorbent Functionalized with N-Methyl-D-Glucamine by Click Chemistry and Removal of Boron with Magnetic Separation Method. Ecotoxicol. Environ. Saf. 2018, 162, 245-252.

(29) Shoghi, E.; Mirahmadi-Zare, S. Z.; Ghasemi, R.; Asghari, M.; Poorebrahim, M.; NasrEsfahani, M.-H. Nanosized Aptameric Cavities Imprinted on the Surface of Magnetic Nanoparticles for High-Throughput Protein Recognition. Mikrochim. Acta 2018, 185, 241.

(30) Finetti, C.; Sola, L.; Pezzullo, M.; Prosperi, D.; Colombo, M.; Riva, B.; Avvakumova, S.; Morasso, C.; Picciolini, S.; Chiari, M. Click Chemistry Immobilization of Antibodies on Polymer Coated Gold Nanoparticles. Langmuir 2016, 32, 7435-7441.

(31) Abadeer, N. S.; Brennan, M. R.; Wilson, W. L.; Murphy, C. J. Distance and Plasmon Wavelength Dependent Fluorescence of Molecules Bound to Silica-Coated Gold Nanorods. ACS Nano 2014, 8, 8392-8406.

(32) Tang, F.; Li, L.; Chen, D. Mesoporous Silica Nanoparticles: Synthesis, Biocompatibility and Drug Delivery. Adv. Mater. 2012, 24, 1504-1534.

(33) Lu, J.; Liong, M.; Zink, J. I.; Tamanoi, F. Mesoporous Silica Nanoparticles as a Delivery System for Hydrophobic Anticancer Drugs. Small 2007, 3, 1341-1346.

(34) De Crozals, G.; Bonnet, R.; Farre, C.; Chaix, C. Nanoparticles with Multiple Properties for Biomedical Applications: A Strategic Guide. Nano Today 2016, 11, 435-463.

(35) Slowing, I.; Viveroescoto, J.; Wu, C.; Lin, V. Mesoporous Silica Nanoparticles as Controlled Release Drug Delivery and Gene Transfection Carriers. Adv. Drug Deliv. Rev. 2008, 60, 1278-1288.

(36) Chen, Z.; Sun, M.; Luo, F.; Xu, K.; Lin, Z.; Zhang, L. Stimulus-Response Click Chemistry Based Aptamer-Functionalized Mesoporous Silica Nanoparticles for Fluorescence Detection of Thrombin. Talanta 2018, 178, 563-568.

(37) Datz, S.; Argyo, C.; Gattner, M.; Weiss, V.; Brunner, K.; Bretzler, J.; von Schirnding, C.; Torrano, A. A.; Spada, F.; Vrabel, M.; et al. Genetically Designed Biomolecular Capping System for Mesoporous Silica Nanoparticles Enables Receptor-Mediated Cell Uptake and Controlled Drug Release. Nanoscale 2016, 8, 8101-8110.

(38) Noureddine, A.; Gary-Bobo, M.; Lichon, L.; Garcia, M.; Zink, J. I.; Wong Chi Man, M.; Cattoën, X. Bis-Clickable Mesoporous Silica Nanoparticles: Straightforward Preparation of Light-Actuated Nanomachines for Controlled Drug Delivery with Active Targeting. Chem. Weinh. Bergstr. Ger. 2016, 22, 9624-9630.

(39) Chertok, B.; Moffat, B. A.; David, A. E.; Yu, F.; Bergemann, C.; Ross, B. D.; Yang, V. C. Iron Oxide Nanoparticles as a Drug Delivery Vehicle for MRI Monitored Magnetic Targeting of Brain Tumors. Biomaterials 2008, 29, 487-496.

(40) Lee, H.-Y.; Li, Z.; Chen, K.; Hsu, A. R.; Xu, C.; Xie, J.; Sun, S.; Chen, X. PET/MRI Dual-Modality Tumor Imaging Using Arginine-Glycine-Aspartic (RGD)-Conjugated Radiolabeled Iron Oxide Nanoparticles. J. Nucl. Med. 2008, 49, 1371-1379.

(41) Xie, J.; Chen, K.; Huang, J.; Lee, S.; Wang, J.; Gao, J.; Li, X.; Chen, X. PET/NIRF/MRI Triple Functional Iron Oxide Nanoparticles. Biomaterials 2010, 31, 3016-3022. 
(42) Massaad-Massade, L.; Boutary, S.; Caillaud, M.; Gracia, C.; Parola, B.; Gnaouiya, S. B.; Stella, B.; Arpicco, S.; Buchy, E.; Desmaële, D.; et al. New Formulation for the Delivery of Oligonucleotides Using "Clickable" SiRNA-Polyisoprenoid-Conjugated Nanoparticles: Application to Cancers Harboring Fusion Oncogenes. Bioconjug. Chem. 2018, 29, 1961-1972.

(43) Mahmoudi, M.; Sant, S.; Wang, B.; Laurent, S.; Sen, T. Superparamagnetic Iron Oxide Nanoparticles (SPIONs): Development, Surface Modification and Applications in Chemotherapy. Adv. Drug Deliv. Rev. 2011, 63, 24-46.

(44) Arriortua, O. K.; Insausti, M.; Lezama, L.; Gil de Muro, I.; Garaio, E.; de la Fuente, J. M.; Fratila, R. M.; Morales, M. P.; Costa, R.; Eceiza, M.; et al. RGD-Functionalized Fe3O4nanoparticles for Magnetic Hyperthermia. Colloids Surf. B Biointerfaces 2018, 165, 315-324.

(45) Meghani, N. M.; Amin, H. H.; Park, C.; Park, J.-B.; Cui, J.-H.; Cao, Q.-R.; Lee, B.-J. Design and Evaluation of Clickable Gelatin-Oleic Nanoparticles Using FattigationPlatform for Cancer Therapy. Int. J. Pharm. 2018, 545, 101-112.

(46) Liu, Y.; Hou, W.; Sun, H.; Cui, C.; Zhang, L.; Jiang, Y.; Wu, Y.; Wang, Y.; Li, J.; Sumerlin, B. S.; et al. Thiol-Ene Click Chemistry: A Biocompatible Way for Orthogonal Bioconjugation of Colloidal Nanoparticles. Chem. Sci. 2017, 8, 61826187.

(47) Neouze, M.-A.; Schubert, U. Surface Modification and Functionalization of Metal and Metal Oxide Nanoparticles by Organic Ligands. Monatshefte Für Chem. - Chem. Mon. 2008, 139, 183-195.

(48) Subbiah, R.; Veerapandian, M.; S. Yun, K. Nanoparticles: Functionalization and Multifunctional Applications in Biomedical Sciences. Curr. Med. Chem. 2010, 17, 4559-4577.

(49) G.T. Hermanson. Bioconjugate Techniques. Acad. Press 2013.

(50) Algar, W. R.; Prasuhn, D. E.; Stewart, M. H.; Jennings, T. L.; Blanco-Canosa, J. B.; Dawson, P. E.; Medintz, I. L. The Controlled Display of Biomolecules on Nanoparticles: A Challenge Suited to Bioorthogonal Chemistry. Bioconjug. Chem. 2011, 22, 825-858.

(51) Nakajima, N.; Ikada, Y. Mechanism of Amide Formation by Carbodiimide for Bioconjugation in Aqueous Media. Bioconjug. Chem. 1995, 6, 123-130.

(52) Rahim, M. K.; Kota, R.; Lee, S.; Haun, J. B. Bioorthogonal Chemistries for Nanomaterial Conjugation and Targeting. Nanotechnol. Rev. 2013, 2, 215-227.

(53) Such, G. K.; Johnston, A. P. R.; Liang, K.; Caruso, F. Synthesis and Functionalization of Nanoengineered Materials Using Click Chemistry. Prog. Polym. Sci. 2012, 37, 985-1003.

(54) Han, H.-S.; Devaraj, N. K.; Lee, J.; Hilderbrand, S. A.; Weissleder, R.; Bawendi, M. G. Development of a Bioorthogonal and Highly Efficient Conjugation Method for Quantum Dots Using Tetrazine-Norbornene Cycloaddition. J. Am. Chem. Soc. 2010, 132, 7838-7839.

(55) Kotagiri, N.; Li, Z.; Xu, X.; Mondal, S.; Nehorai, A.; Achilefu, S. Antibody Quantum Dot Conjugates Developed via Copper-Free Click Chemistry for Rapid Analysis of Biological Samples Using a Microfluidic Microsphere Array System. Bioconjug. Chem. 2014, 25, 1272-1281.

(56) Kaplun, V.; Stepensky, D. Efficient Decoration of Nanoparticles Intended for Intracellular Drug Targeting with Targeting Residues, as Revealed by a New Indirect Analytical Approach. Mol. Pharm. 2014, 11, 2906-2914.

(57) Williams, S.; Neumann, A.; Bremer, I.; Su, Y.; Dräger, G.; Kasper, C.; Behrens, P. Nanoporous Silica Nanoparticles as Biomaterials: Evaluation of Different Strategies for the Functionalization with Polysialic Acid by Step-by-Step Cytocompatibility Testing. J. Mater. Sci. Mater. Med. 2015, 26, 125-131.

(58) Jewett, J. C.; Bertozzi, C. R. Cu-Free Click Cycloaddition Reactions in Chemical Biology. Chem. Soc. Rev. 2010, 39, 1272-1279.

(59) Dommerholt, J.; van Rooijen, O.; Borrmann, A.; Guerra, C. F.; Bickelhaupt, F. M.; van Delft, F. L. Highly Accelerated Inverse Electron-Demand Cycloaddition of ElectronDeficient Azides with Aliphatic Cyclooctynes. Nat. Commun. 2014, 5, 5378-5384. 
(60) Korthals, B.; Morant-Miñana, M. C.; Schmid, M.; Mecking, S. Functionalization of Polymer Nanoparticles by Thiol-Ene Addition. Macromolecules 2010, 43, 80718078.

(61) Ruizendaal, L.; Pujari, S. P.; Gevaerts, V.; Paulusse, J. M. J.; Zuilhof, H. Biofunctional Silicon Nanoparticles by Means of Thiol-Ene Click Chemistry. Chem. Asian J. 2011, 6, 2776-2786.

(62) Hoyle, C. E.; Bowman, C. N. Thiol-Ene Click Chemistry. Angew. Chem. Int. Ed. 2010, 49, 1540-1573.

(63) Hoyle, C. E.; Lowe, A. B.; Bowman, C. N. Thiol-Click Chemistry: A Multifaceted Toolbox for Small Molecule and Polymer Synthesis. Chem. Soc. Rev. 2010, 39, 1355-1387.

(64) Stéen, E. J. L.; Edem, P. E.; Nørregaard, K.; Jørgensen, J. T.; Shalgunov, V.; Kjaer, A.; Herth, M. M. Pretargeting in Nuclear Imaging and Radionuclide Therapy: Improving Efficacy of Theranostics and Nanomedicines. Biomaterials 2018, 179, 209-245.

(65) Devaraj, N. K.; Weissleder, R. Biomedical Applications of Tetrazine Cycloadditions. Acc. Chem. Res. 2011, 44, 816-827.

(66) Gregoritza, M.; Brandl, F. P. The Diels-Alder Reaction: A Powerful Tool for the Design of Drug Delivery Systems and Biomaterials. Eur. J. Pharm. Biopharm. Off. J. Arbeitsgemeinschaft Pharm. Verfahrenstechnik EV 2015, 97, 438-453.

(67) Yi, G.; Son, J.; Yoo, J.; Park, C.; Koo, H. Application of Click Chemistry in Nanoparticle Modification and Its Targeted Delivery. Biomater. Res. 2018, 22-29.

(68) Matsumura, Y.; Maeda, H. A New Concept for Macromolecular Therapeutics in Cancer Chemotherapy: Mechanism of Tumoritropic Accumulation of Proteins and the Antitumor Agent Smancs. Cancer Res. 1986, 46, 6387-6392.

(69) Dai, Y.; Xu, C.; Sun, X.; Chen, X. Nanoparticle Design Strategies for Enhanced Anticancer Therapy by Exploiting the Tumour Microenvironment. Chem Soc Rev 2017, 3830-3852.

(70) Kobayashi, H.; Watanabe, R.; Choyke, P. L. Improving Conventional Enhanced Permeability and Retention (EPR) Effects; What Is the Appropriate Target? Theranostics 2014, 4, 81-89.

(71) Barenholz, Y. (Chezy). Doxil® - The First FDA-Approved Nano-Drug: Lessons Learned. J. Controlled Release 2012, 160, 117-134.

(72) Miele, E.; Spinelli, G. P.; Miele, E.; Tomao, F.; Tomao, S. Albumin-Bound Formulation of Paclitaxel (Abraxane ABI-007) in the Treatment of Breast Cancer. Int. J. Nanomedicine 2009, 4, 99-105.

(73) Zhang, K.; Tang, X.; Zhang, J.; Lu, W.; Lin, X.; Zhang, Y.; Tian, B.; Yang, H.; He, H. PEG-PLGA Copolymers: Their Structure and Structure-Influenced Drug Delivery Applications. J. Controlled Release 2014, 183, 77-86.

(74) Rosenblum, D.; Joshi, N.; Tao, W.; Karp, J. M.; Peer, D. Progress and Challenges towards Targeted Delivery of Cancer Therapeutics. Nat. Commun. 2018, 9, 1410.

(75) Mei, L.; Rao, J.; Liu, Y.; Li, M.; Zhang, Z.; He, Q. Effective Treatment of the Primary Tumor and Lymph Node Metastasis by Polymeric Micelles with Variable Particle Sizes. J. Control. Release Off. J. Control. Release Soc. 2018, 292, 67-77.

(76) Mei, L.; Liu, Y.; Rao, J.; Tang, X.; Li, M.; Zhang, Z.; He, Q. Enhanced Tumor Retention Effect by Click Chemistry for Improved Cancer Immunochemotherapy. ACS Appl. Mater. Interfaces 2018, 10, 17582-17593.

(77) Toomari, Y.; Namazi, H.; Akbar, E. A. Synthesis of the Dendritic Type $\beta$-Cyclodextrin on Primary Face via Click Reaction Applicable as Drug Nanocarrier. Carbohydr. Polym. 2015, 132, 205-213.

(78) Denis, I.; El Bahhaj, F.; Collette, F.; Delatouche, R.; Gueugnon, F.; Pouliquen, D.; Pichavant, L.; Héroguez, V.; Grégoire, M.; Bertrand, P.; et al. Vorinostat-Polymer Conjugate Nanoparticles for Acid-Responsive Delivery and Passive Tumor Targeting. Biomacromolecules 2014, 15, 4534-4543.

(79) Yu, Y.; Chen, C.-K.; Law, W.-C.; Weinheimer, E.; Sengupta, S.; Prasad, P. N.; Cheng, C. Polylactide-Graft-Doxorubicin Nanoparticles with Precisely Controlled Drug Loading for PH-Triggered Drug Delivery. Biomacromolecules 2014, 15, 524-532. 
(80) Zhu, C.; Xiao, J.; Tang, M.; Feng, H.; Chen, W.; Du, M. Platinum Covalent Shell Cross-Linked Micelles Designed to Deliver Doxorubicin for Synergistic Combination Cancer Therapy. Int. J. Nanomedicine 2017, 12, 3697-3710.

(81) Noureddine, A.; Lichon, L.; Maynadier, M.; Garcia, M.; Gary-Bobo, M.; Zink, J. I.; Cattoën, X.; Wong Chi Man, M. Controlled Multiple Functionalization of Mesoporous Silica Nanoparticles: Homogeneous Implementation of Pairs of Functionalities Communicating through Energy or Proton Transfers. Nanoscale 2015, 7, 1144411452.

(82) Cai, K.; Yen, J.; Yin, Q.; Liu, Y.; Song, Z.; Lezmi, S.; Zhang, Y.; Yang, X.; Helferich, W. G.; Cheng, J. Redox-Responsive Self-Assembled Chain-Shattering Polymeric Therapeutics. Biomater. Sci. 2015, 3, 1061-1065.

(83) Abánades Lázaro, I.; Haddad, S.; Sacca, S.; Orellana-Tavra, C.; Fairen-Jimenez, D.; Forgan, R. S. Selective Surface PEGylation of UiO-66 Nanoparticles for Enhanced Stability, Cell Uptake, and PH-Responsive Drug Delivery. Chem 2017, 2, 561-578.

Mai, K.; Lin, J.; Zhuang, B.; Li, X.; Zhang, L.-M. Cationic Dendronization of Amylose via Click Chemistry for Complexation and Transfection of Plasmid DNA. Int. J. Biol. Macromol. 2015, 79, 209-216.

Mai, K.; Zhang, S.; Liang, B.; Gao, C.; Du, W.; Zhang, L.-M. Water Soluble Cationic Dextran Derivatives Containing Poly(Amidoamine) Dendrons for Efficient Gene Delivery. Carbohydr. Polym. 2015, 123, 237-245.

Chen, R.; Zhunang, B.; Li, Z.; Li, W.; Huang, P.; Pang, J.; Zhou, Y.; Lin, Q.; Zhou, Q.; Ye, X.; et al. Nanocomplexation of Thrombin with Cationic Amylose Derivative for Improved Stability And Hemostatic Efficacy. Int. J. Nanomedicine 2015, 939-947.

Yan, Y.; Fu, J.; Wang, T.; Lu, X. Controlled Release of Silyl Ether Camptothecin from Thiol-Ene Click Chemistry-Functionalized Mesoporous Silica Nanoparticles. Acta Biomater. 2017, 51, 471-478.

Cai, L.; Xu, G.; Shi, C.; Guo, D.; Wang, X.; Luo, J. Telodendrimer Nanocarrier for CoDelivery of Paclitaxel and Cisplatin: A Synergistic Combination Nanotherapy for Ovarian Cancer Treatment. Biomaterials 2015, 37, 456-468.

He, H.; Xiao, H.; Kuang, H.; Xie, Z.; Chen, X.; Jing, X.; Huang, Y. Synthesis of Mesoporous Silica Nanoparticle-Oxaliplatin Conjugates for Improved Anticancer Drug Delivery. Colloids Surf. B Biointerfaces 2014, 117, 75-81.

Eroglu, S., M.; Toksoy Oner, E.; Cansever Mutlu, E.; Sennaroglu Bostan, M. Sugar Based Biopolymers in Nanomedicine; New Emerging Era for Cancer Imaging and Therapy. Curr. Top. Med. Chem. 2017, 17, 1507-1520.

Bacinello, D.; Garanger, E.; Taton, D.; Tam, K. C.; Lecommandoux, S. EnzymeDegradable Self-Assembled Nanostructures from Polymer-Peptide Hybrids. Biomacromolecules 2014, 15, 1882-1888.

Kim, H. S.; Yoon, S.; Son, Y. J.; Park, Y.; Jung, Y. M.; Yoo, H. S. High-Yield Clicking and Dissociation of Doxorubicin Nanoclusters Exhibiting Differential Cellular Uptakes and Imaging. J. Control. Release Off. J. Control. Release Soc. 2015, 217, 64-73.

Zhang, C.; Pan, D.; Li, J.; Hu, J.; Bains, A.; Guys, N.; Zhu, H.; Li, X.; Luo, K.; Gong, et al. Enzyme-Responsive Peptide Dendrimer-Gemcitabine Conjugate as a Controlled-Release Drug Delivery Vehicle with Enhanced Antitumor Efficacy. Acta Biomater. 2017, 55, 153-162.

Li, N.; Li, N.; Yi, Q.; Luo, K.; Guo, C.; Pan, D.; Gu, Z. Amphiphilic Peptide Dendritic Copolymer-Doxorubicin Nanoscale Conjugate Self-Assembled to EnzymeResponsive Anti-Cancer Agent. Biomaterials 2014, 35, 9529-9545.

Sharma, R.; Kim, S.-Y.; Sharma, A.; Zhang, Z.; Kambhampati, S. P.; Kannan, S.; Kannan, R. M. Activated Microglia Targeting Dendrimer-Minocycline Conjugate as Therapeutics for Neuroinflammation. Bioconjug. Chem. 2017, 28, 2874-2886.

Jiang, P.; Li, S.; Lai, J.; Zheng, H.; Lin, C.; Shi, P.; Wang, Y. NanoparticleProgrammed Surface for Drug Release and Cell Regulation via Reversible Hybridization Reaction. ACS Appl. Mater. Interfaces 2017, 9, 4467-4474.

Denis, I.; el Bahhaj, F.; Collette, F.; Delatouche, R.; Gueugnon, F.; Pouliquen, D.; Pichavant, L.; Héroguez, V.; Grégoire, M.; Bertrand, P.; et al. Histone Deacetylase 
Inhibitor-Polymer Conjugate Nanoparticles for Acid-Responsive Drug Delivery. Eur. J. Med. Chem. 2015, 95, 369-376.

(98) El Bahhaj, F.; Denis, I.; Pichavant, L.; Delatouche, R.; Collette, F.; Linot, C.; Pouliquen, D.; Grégoire, M.; Héroguez, V.; Blanquart, C.; et al. Histone Deacetylase Inhibitors Delivery Using Nanoparticles with Intrinsic Passive Tumor Targeting Properties for Tumor Therapy. Theranostics 2016, 6, 795-807.

(99) Xu, L.; Zolotarskaya, O. Y.; Yeudall, W. A.; Yang, H. Click Hybridization of Immune Cells and Polyamidoamine Dendrimers. Adv. Healthc. Mater. 2014, 3, 1430-1438.

(100) Gondi, C. S.; Rao, J. S. Cathepsin B as a Cancer Target. Expert Opin. Ther. Targets 2013, 17, 281-291.

(101) Lyu, Y.; Zhen, X.; Miao, Y.; Pu, K. Reaction-Based Semiconducting Polymer Nanoprobes for Photoacoustic Imaging of Protein Sulfenic Acids. ACS Nano 2017, 11, 358-367.

(102) Xing, Y.; Zhu, J.; Zhao, L.; Xiong, Z.; Li, Y.; Wu, S.; Chand, G.; Shi, X.; Zhao, J. SPECT/CT Imaging of Chemotherapy-Induced Tumor Apoptosis Using 99mTcLabeled Dendrimer-Entrapped Gold Nanoparticles. Drug Deliv. 2018, 25, 1384-1393.

(103) Wei, Z.; Wu, M.; Li, Z.; Lin, Z.; Zeng, J.; Sun, H.; Liu, X.; Liu, J.; Li, B.; Zeng, Y. Gadolinium-Doped Hollow CeO2-ZrO2 Nanoplatform as Multifunctional MRI/CT DualModal Imaging Agent and Drug Delivery Vehicle. Drug Deliv. 2018, 25, 353-363.

(104) Zhou, B.; Xiong, Z.; Wang, P.; Peng, C.; Shen, M.; Mignani, S.; Majoral, J.-P.; Shi, X. Targeted Tumor Dual Mode CT/MR Imaging Using Multifunctional PolyethylenimineEntrapped Gold Nanoparticles Loaded with Gadolinium. Drug Deliv. 2018, 25, 178186.

(105) He, W.; Cheng, L.; Zhang, L.; Jiang, X.; Liu, Z.; Cheng, Z.; Zhu, X. Bifunctional Nanoparticles with Magnetism and NIR Fluorescence: Controlled Synthesis from Combination of AGET ATRP and "click" Reaction. Nanotechnology 2014, 25, 045602.

(106) Teske, N. S.; Voigt, J.; Shastri, V. P. Clickable Degradable Aliphatic Polyesters via Copolymerization with Alkyne Epoxy Esters: Synthesis and Postfunctionalization with Organic Dyes. J. Am. Chem. Soc. 2014, 136, 10527-10533.

(107) Hollingsworth, J. V.; Bhupathiraju, N. V. S. D. K.; Sun, J.; Lochner, E.; Vicente, M. G. H.; Russo, P. S. Preparation of Metalloporphyrin-Bound Superparamagnetic Silica Particles via "Click" Reaction. ACS Appl. Mater. Interfaces 2016, 8, 792-801.

(108) Lee, S.; Kang, S.-W.; Ryu, J. H.; Na, J. H.; Lee, D.-E.; Han, S. J.; Kang, C. M.; Choe, Y. S.; Lee, K. C.; Leary, J. F.; et al. Tumor-Homing Glycol Chitosan-Based Optical/PET Dual Imaging Nanoprobe for Cancer Diagnosis. Bioconjug. Chem. 2014, 25, 601-610.

(109) Pérez-Medina, C.; Abdel-Atti, D.; Zhang, Y.; Longo, V. A.; Irwin, C. P.; Binderup, T.; Ruiz-Cabello, J.; Fayad, Z. A.; Lewis, J. S.; Mulder, W. J. M.; et al. A Modular Labeling Strategy for in vivo PET and Near-Infrared Fluorescence Imaging of Nanoparticle Tumor Targeting. J. Nucl. Med. Off. Publ. Soc. Nucl. Med. 2014, 55, 1706-1711.

(110) Jeon, J.; Kang, J. A.; Shim, H. E.; Nam, Y. R.; Yoon, S.; Kim, H. R.; Lee, D. E.; Park, S. H. Efficient Method for lodine Radioisotope Labeling of Cyclooctyne-Containing Molecules Using Strain-Promoted Copper-Free Click Reaction. Bioorg. Med. Chem. 2015, 23, 3303-3308.

(111) Arranja, A. G.; Pathak, V.; Lammers, T.; Shi, Y. Tumor-Targeted Nanomedicines for Cancer Theranostics. Pharmacol. Res. 2017, 115, 87-95.

(112) Lammers, T.; Kiessling, F.; Hennink, W. E.; Storm, G. Drug Targeting to Tumors: Principles, Pitfalls and (Pre-) Clinical Progress. J. Controlled Release 2012, 161, 175-187.

(113) Sun, Q.; Ojha, T.; Kiessling, F.; Lammers, T.; Shi, Y. Enhancing Tumor Penetration of Nanomedicines. Biomacromolecules 2017, 18, 1449-1459.

(114) Golombek, S. K.; May, J.-N.; Theek, B.; Appold, L.; Drude, N.; Kiessling, F.; Lammers, T. Tumor Targeting via EPR: Strategies to Enhance Patient Responses. Adv. Drug Deliv. Rev. 2018, 130, 17-38. 
(115) Salatin, S.; Maleki Dizaj, S.; Yari Khosroushahi, A. Effect of the Surface Modification, Size, and Shape on Cellular Uptake of Nanoparticles. Cell Biol. Int. 2015, 39, 881890.

(116) Albanese, A.; Tang, P. S.; Chan, W. C. W. The Effect of Nanoparticle Size, Shape, and Surface Chemistry on Biological Systems. Annu. Rev. Biomed. Eng. 2012, 14, 116.

(117) Danhier, F. To Exploit the Tumor Microenvironment: Since the EPR Effect Fails in the Clinic, What Is the Future of Nanomedicine? J. Controlled Release 2016, 244, 108121.

(118) Sulthana, S.; Banerjee, T.; Kallu, J.; Vuppala, S. R.; Heckert, B.; Naz, S.; Shelby, T.; Yambem, O.; Santra, S. Combination Therapy of NSCLC Using Hsp90 Inhibitor and Doxorubicin Carrying Functional Nanoceria. Mol. Pharm. 2017, 14, 875-884.

(119) Junyaprasert, V. B.; Dhanahiranpruk, S.; Suksiriworapong, J.; Sripha, K.; Moongkarndi, P. Enhanced Toxicity and Cellular Uptake of Methotrexate-Conjugated Nanoparticles in Folate Receptor-Positive Cancer Cells by Decorating with Folic AcidConjugated d-a-Tocopheryl Polyethylene Glycol 1000 Succinate. Colloids Surf. B Biointerfaces 2015, 136, 383-393.

(120) Wu, Y.; Zhang, Y.; Zhang, W.; Sun, C.; Wu, J.; Tang, J. Reversing of Multidrug Resistance Breast Cancer by Co-Delivery of P-Gp SiRNA and Doxorubicin via Folic Acid-Modified Core-Shell Nanomicelles. Colloids Surf. B Biointerfaces 2016, 138, 6069.

(121) Singh, Y.; Durga Rao Viswanadham, K. K.; Kumar Jajoriya, A.; Meher, J. G.; Raval, K.; Jaiswal, S.; Dewangan, J.; Bora, H. K.; Rath, S. K.; Lal, J.; et al. Click Biotinylation of PLGA Template for Biotin Receptor Oriented Delivery of Doxorubicin Hydrochloride in 4T1 Cell-Induced Breast Cancer. Mol. Pharm. 2017, 14, 2749-2765.

(122) Liu, R.; Zhao, J.; Han, G.; Zhao, T.; Zhang, R.; Liu, B.; Liu, Z.; Zhang, C.; Yang, L.; Zhang, Z. Click-Functionalized SERS Nanoprobes with Improved Labeling Efficiency and Capability for Cancer Cell Imaging. ACS Appl. Mater. Interfaces 2017, 9, 3822238229.

(123) Olejniczak, J.; Collet, G.; Nguyen Huu, V. A.; Chan, M.; Lee, S.; Almutairi, A. Biorthogonal Click Chemistry on Poly(Lactic-Co-Glycolic Acid)-Polymeric Particles. Biomater. Sci. 2017, 5, 211-215.

(124) Sun, Q.; Kang, Z.; Xue, L.; Shang, Y.; Su, Z.; Sun, H.; Ping, Q.; Mo, R.; Zhang, C. A Collaborative Assembly Strategy for Tumor-Targeted SiRNA Delivery. J. Am. Chem. Soc. 2015, 137, 6000-6010.

(125) Li, H.; Mu, Y.; Qian, S.; Lu, J.; Wan, Y.; Fu, G.; Liu, S. Synthesis of Fluorescent DyeDoped Silica Nanoparticles for Target-Cell-Specific Delivery and Intracellular MicroRNA Imaging. The Analyst 2015, 140, 567-573.

(126) Morris, W.; Briley, W. E.; Auyeung, E.; Cabezas, M. D.; Mirkin, C. A. Nucleic AcidMetal Organic Framework (MOF) Nanoparticle Conjugates. J. Am. Chem. Soc. 2014, 136, 7261-7264.

(127) Wang, C.-F.; Mäkilä, E. M.; Bonduelle, C.; Rytkönen, J.; Raula, J.; Almeida, S.; Närvänen, A.; Salonen, J. J.; Lecommandoux, S.; Hirvonen, J. T.; et al. Functionalization of Alkyne-Terminated Thermally Hydrocarbonized Porous Silicon Nanoparticles With Targeting Peptides and Antifouling Polymers: Effect on the Human Plasma Protein Adsorption. ACS Appl. Mater. Interfaces 2015, 7, 2006-2015.

(128) Jia, G.; Han, Y.; An, Y.; Ding, Y.; He, C.; Wang, X.; Tang, Q. NRP-1 Targeted and Cargo-Loaded Exosomes Facilitate Simultaneous Imaging and Therapy of Glioma in vitro and in vivo. Biomaterials 2018, 178, 302-316.

(129) Ke, W.; Zha, Z.; Mukerabigwi, J. F.; Chen, W.; Wang, Y.; He, C.; Ge, Z. Matrix Metalloproteinase-Responsive Multifunctional Peptide-Linked Amphiphilic Block Copolymers for Intelligent Systemic Anticancer Drug Delivery. Bioconjug. Chem. 2017, 28, 2190-2198.

(130) Arriortua, O. K.; Garaio, E.; Herrero de la Parte, B.; Insausti, M.; Lezama, L.; Plazaola, F.; García, J. A.; Aizpurua, J. M.; Sagartzazu, M.; Irazola, M.; et al. Antitumor Magnetic Hyperthermia Induced by RGD-Functionalized $\mathrm{Fe} 3 \mathrm{O} 4$ 
Nanoparticles, in an Experimental Model of Colorectal Liver Metastases. Beilstein J. Nanotechnol. 2016, 7, 1532-1542.

(131) Slegerova, J.; Hajek, M.; Rehor, I.; Sedlak, F.; Stursa, J.; Hruby, M.; Cigler, P. Designing the Nanobiointerface of Fluorescent Nanodiamonds: Highly Selective Targeting of Glioma Cancer Cells. Nanoscale 2015, 7, 415-420.

(132) Yuan, H.; Wilks, M. Q.; El Fakhri, G.; Normandin, M. D.; Kaittanis, C.; Josephson, L. Heat-Induced-Radiolabeling and Click Chemistry: A Powerful Combination for Generating Multifunctional Nanomaterials. PloS One 2017, 12, e0172722.

(133) Qin, M.; Zong, H.; Kopelman, R. Click Conjugation of Peptide to Hydrogel Nanoparticles for Tumor-Targeted Drug Delivery. Biomacromolecules 2014, 15, 3728-3734.

(134) Wang, C.-F.; Sarparanta, M. P.; Mäkilä, E. M.; Hyvönen, M. L. K.; Laakkonen, P. M.; Salonen, J. J.; Hirvonen, J. T.; Airaksinen, A. J.; Santos, H. A. Multifunctional Porous Silicon Nanoparticles for Cancer Theranostics. Biomaterials 2015, 48, 108-118.

(135) Wang, C.-F.; Mäkilä, E. M.; Kaasalainen, M. H.; Liu, D.; Sarparanta, M. P.; Airaksinen, A. J.; Salonen, J. J.; Hirvonen, J. T.; Santos, H. A. Copper-Free AzideAlkyne Cycloaddition of Targeting Peptides to Porous Silicon Nanoparticles for Intracellular Drug Uptake. Biomaterials 2014, 35, 1257-1266.

(136) Huang, B.; Otis, J.; Joice, M.; Kotlyar, A.; Thomas, T. P. PSMA-Targeted Stably Linked "Dendrimer-Glutamate Urea-Methotrexate" as a Prostate Cancer Therapeutic. Biomacromolecules 2014, 15, 915-923.

(137) Zhao, M.; Liu, Y.; Hsieh, R. S.; Wang, N.; Tai, W.; Joo, K.-I.; Wang, P.; Gu, Z.; Tang, Y. Clickable Protein Nanocapsules for Targeted Delivery of Recombinant P53 Protein. J. Am. Chem. Soc. 2014, 136, 15319-15325.

(138) Otis, J. B.; Zong, H.; Kotylar, A.; Yin, A.; Bhattacharjee, S.; Wang, H.; Baker, J. R.; Wang, S. H. Dendrimer Antibody Conjugate to Target and Image HER-2 Overexpressing Cancer Cells. Oncotarget 2016, 7, 36002-36013.

(139) Zhou, Z.; Badkas, A.; Stevenson, M.; Lee, J.-Y.; Leung, Y.-K. Herceptin Conjugated PLGA-PHis-PEG PH Sensitive Nanoparticles for Targeted and Controlled Drug Delivery. Int. J. Pharm. 2015, 487, 81-90.

(140) Lin, Q.; Yang, Y.; Hu, Q.; Guo, Z.; Liu, T.; Xu, J.; Wu, J.; Kirk, T. B.; Ma, D.; Xue, W. Injectable Supramolecular Hydrogel Formed from a-Cyclodextrin and PEGylated Arginine-Functionalized Poly(I-Lysine) Dendron for Sustained MMP-9 ShRNA Plasmid Delivery. Acta Biomater. 2017, 49, 456-471.

(141) Li, X.; Zhao, L.; Liang, Q.; Liang, Q.; Ye, J.; Komatsu, N.; Zhang, Q.; Gao, W.; Xu, M.; Chen, X. Cationic Polyarginine Conjugated Mesoporous Bioactive Glass Nanoparticles with Polyglycerol Coating for Efficient DNA Delivery. J. Biomed. Nanotechnol. 2017, 13, 280-289.

(142) Maity, A. R.; Stepensky, D. Efficient Subcellular Targeting to the Cell Nucleus of Quantum Dots Densely Decorated with a Nuclear Localization Sequence Peptide. ACS Appl. Mater. Interfaces 2016, 8, 2001-2009.

(143) Tatiparti, K.; Sau, S.; Gawde, K. A.; Iyer, A. K. Copper-Free "Click" Chemistry-Based Synthesis and Characterization of Carbonic Anhydrase-IX Anchored AlbuminPaclitaxel Nanoparticles for Targeting Tumor Hypoxia. Int. J. Mol. Sci. 2018, 19. 838.

(144) Calabrese, C. M.; Merkel, T. J.; Briley, W. E.; Randeria, P. S.; Narayan, S. P.; Rouge, J. L.; Walker, D. A.; Scott, A. W.; Mirkin, C. A. Biocompatible Infinite-CoordinationPolymer Nanoparticle-Nucleic-Acid Conjugates for Antisense Gene Regulation. Angew. Chem. Int. Ed Engl. 2015, 54, 476-480.

(145) Collina, S. New Perspectives in Cancer Therapy: The Biotin-Antitumor Molecule Conjugates. Med. Chem. 2014, S1, 1-8.

(146) Zwicke, G. L.; Ali Mansoori, G.; Jeffery, C. J. Utilizing the Folate Receptor for Active Targeting of Cancer Nanotherapeutics. Nano Rev. 2012, 3, 18496.

(147) Puvvada, N.; Rajput, S.; Kumar, B. N. P.; Sarkar, S.; Konar, S.; Brunt, K. R.; Rao, R. R.; Mazumdar, A.; Das, S. K.; Basu, R.; et al. Novel ZnO Hollow-Nanocarriers Containing Paclitaxel Targeting Folate-Receptors in a Malignant $\mathrm{PH}$ Microenvironment for Effective Monitoring and Promoting Breast Tumor Regression. Sci. Rep. 2015, 5, 11760. 
(148) Wei, J.; Shuai, X.; Wang, R.; He, X.; Li, Y.; Ding, M.; Li, J.; Tan, H.; Fu, Q. Clickable and Imageable Multiblock Polymer Micelles with Magnetically Guided and PEGSwitched Targeting and Release Property for Precise Tumor Theranosis. Biomaterials 2017, 145, 138-153.

(149) Zhang, Y.; Lv, T.; Zhang, H.; Xie, X.; Li, Z.; Chen, H.; Gao, Y. Folate and Heptamethine Cyanine Modified Chitosan-Based Nanotheranostics for Tumor Targeted Near-Infrared Fluorescence Imaging and Photodynamic Therapy. Biomacromolecules 2017, 18, 2146-2160.

(150) Guldris, N.; Gallo, J.; García-Hevia, L.; Rivas, J.; Bañobre-López, M.; Salonen, L. M. Orthogonal Clickable Iron Oxide Nanoparticle Platform for Targeting, Imaging, and On-Demand Release. Chem. Weinh. Bergstr. Ger. 2018, 24, 8624-8631.

(151) Kato, D.; Oishi, M. Ultrasensitive Detection of DNA and RNA Based on Enzyme-Free Click Chemical Ligation Chain Reaction on Dispersed Gold Nanoparticles. ACS Nano 2014, 8, 9988-9997.

(152) Mendez-Gonzalez, D.; Laurenti, M.; Latorre, A.; Somoza, A.; Vazquez, A.; Negredo, A. I.; López-Cabarcos, E.; Calderón, O. G.; Melle, S.; Rubio-Retama, J. Oligonucleotide Sensor Based on Selective Capture of Upconversion Nanoparticles Triggered by Target-Induced DNA Interstrand Ligand Reaction. ACS Appl. Mater. Interfaces 2017, 9, 12272-12281.

(153) Rambaruth, N. D. S.; Dwek, M. V. Cell Surface Glycan-Lectin Interactions in Tumor Metastasis. Acta Histochem. 2011, 113, 591-600.

(154) Nakahara, S.; Raz, A. Biological Modulation by Lectins and Their Ligands in Tumor Progression and Metastasis. Anticancer Agents Med. Chem. 2008, 8, 22-36.

(155) Poonthiyil, V.; Lindhorst, T. K.; Golovko, V. B.; Fairbanks, A. J. Recent Applications of Click Chemistry for the Functionalization of Gold Nanoparticles and Their Conversion to Glyco-Gold Nanoparticles. Beilstein J. Org. Chem. 2018, 14, 11-24.

(156) Choi, K. Y.; Yoon, H. Y.; Kim, J.-H.; Bae, S. M.; Park, R.-W.; Kang, Y. M.; Kim, I.-S.; Kwon, I. C.; Choi, K.; Jeong, S. Y.; et al. Smart Nanocarrier Based on PEGylated Hyaluronic Acid for Cancer Therapy. ACS Nano 2011, 5, 8591-8599.

(157) Lundquist, J. J.; Toone, E. J. The Cluster Glycoside Effect. Chem. Rev. 2002, 102, 555-578.

(158) Bezouska, K. Design, Functional Evaluation and Biomedical Applications of Carbohydrate Dendrimers (Glycodendrimers). J. Biotechnol. 2002, 90, 269-290.

(159) Rajakumar, P.; Anandhan, R.; Vadla, G. P.; Vellaichamy, E. Synthesis and Cardio Protective Biological Applications of Glucodendrimers by H9C2 Cell Studies. Carbohydr. Polym. 2014, 99, 403-414.

(160) Kong, N.; Zhou, J.; Park, J.; Xie, S.; Ramström, O.; Yan, M. Quantitative Fluorine NMR to Determine Carbohydrate Density on Glyconanomaterials Synthesized from Perfluorophenyl Azide-Functionalized Silica Nanoparticles by Click Reaction. Anal. Chem. 2015, 87, 9451-9458.

(161) Tollas, S.; Bereczki, I.; Borbás, A.; Batta, G.; Vanderlinden, E.; Naesens, L.; Herczegh, P. Synthesis of a Cluster-Forming Sialylthio-D-Galactose Fullerene Conjugate and Evaluation of Its Interaction with Influenza Virus Hemagglutinin and Neuraminidase. Bioorg. Med. Chem. Lett. 2014, 24, 2420-2423.

(162) Keefe, A. D.; Pai, S.; Ellington, A. Aptamers as Therapeutics. Nat. Rev. Drug Discov. 2010, 9, 537-550.

(163) Ma, L.; Tu, C.; Le, P.; Chitoor, S.; Lim, S. J.; Zahid, M. U.; Teng, K. W.; Ge, P.; Selvin, P. R.; Smith, A. M. Multidentate Polymer Coatings for Compact and Homogeneous Quantum Dots with Efficient Bioconjugation. J. Am. Chem. Soc. 2016, 138, 3382-3394.

(164) Chen, W.-H.; Yu, X.; Cecconello, A.; Sohn, Y. S.; Nechushtai, R.; Willner, I. StimuliResponsive Nucleic Acid-Functionalized Metal-Organic Framework Nanoparticles Using PH- and Metal-Ion-Dependent DNAzymes as Locks. Chem. Sci. 2017, 8, 5769-5780.

(165) Ding, Q.; Zhan, Q.; Zhou, X.; Zhang, T.; Xing, D. Theranostic Upconversion Nanobeacons for Tumor MRNA Ratiometric Fluorescence Detection and ImagingMonitored Drug Delivery. Small Weinh. Bergstr. Ger. 2016, 12, 5944-5953. 
(166) Akiel, R. D.; Zhang, X.; Abeywardana, C.; Stepanov, V.; Qin, P. Z.; Takahashi, S. Investigating Functional DNA Grafted on Nanodiamond Surface Using Site-Directed Spin Labeling and Electron Paramagnetic Resonance Spectroscopy. J. Phys. Chem. B 2016, 120, 4003-4008.

(167) Barczyk, M.; Carracedo, S.; Gullberg, D. Integrins. Cell Tissue Res. 2010, 339, 269280.

(168) Thumshirn, G.; Hersel, U.; Goodman, S. L.; Kessler, H. Multimeric Cyclic RGD Peptides as Potential Tools for Tumor Targeting: Solid-Phase Peptide Synthesis and Chemoselective Oxime Ligation. Chem. - Eur. J. 2003, 9, 2717-2725.

(169) Rehor, I.; Slegerova, J.; Kucka, J.; Proks, V.; Petrakova, V.; Adam, M.-P.; Treussart, F.; Turner, S.; Bals, S.; Sacha, P.; et al. Fluorescent Nanodiamonds Embedded in Biocompatible Translucent Shells. Small Weinh. Bergstr. Ger. 2014, 10, 1106-1115.

(170) Oz, Y.; Arslan, M.; Gevrek, T. N.; Sanyal, R.; Sanyal, A. Modular Fabrication of Polymer Brush Coated Magnetic Nanoparticles: Engineering the Interface for Targeted Cellular Imaging. ACS Appl. Mater. Interfaces 2016, 8, 19813-19826.

(171) Lindgren, M.; Hällbrink, M.; Prochiantz, A.; Langel, Ü. Cell-Penetrating Peptides. Trends Pharmacol. Sci. 2000, 21, 99-103.

(172) Vivès, E.; Brodin, P.; Lebleu, B. A. Truncated HIV-1 Tat Protein Basic Domain Rapidly Translocates through the Plasma Membrane and Accumulates in the Cell Nucleus. J. Biol. Chem. 1997, 272, 16010-16017.

(173) Liu, D.; Zhang, H.; Mäkilä, E.; Fan, J.; Herranz-Blanco, B.; Wang, C.-F.; Rosa, R.; Ribeiro, A. J.; Salonen, J.; Hirvonen, J.; et al. Microfluidic Assisted One-Step Fabrication of Porous Silicon@acetalated Dextran Nanocomposites for Precisely Controlled Combination Chemotherapy. Biomaterials 2015, 39, 249-259.

(174) Han, S.-S.; Li, Z.-Y.; Zhu, J.-Y.; Han, K.; Zeng, Z.-Y.; Hong, W.; Li, W.-X.; Jia, H.-Z.; Liu, Y.; Zhuo, R.-X.; et al. Dual-PH Sensitive Charge-Reversal Polypeptide Micelles for Tumor-Triggered Targeting Uptake and Nuclear Drug Delivery. Small Weinh. Bergstr. Ger. 2015, 11, 2543-2554.

(175) Brunner, K.; Harder, J.; Halbach, T.; Willibald, J.; Spada, F.; Gnerlich, F.; Sparrer, K.; Beil, A.; Möckl, L.; Bräuchle, C.; et al. Cell-Penetrating and Neurotargeting Dendritic SiRNA Nanostructures. Angew. Chem. Int. Ed Engl. 2015, 54, 1946-1949.

(176) Wan, J.; Brust, A.; Bhola, R. F.; Jha, P.; Mobli, M.; Lewis, R. J.; Christie, M. J.; Alewood, P. F. Inhibition of the Norepinephrine Transporter by X-Conotoxin Dendrimers. J. Pept. Sci. Off. Publ. Eur. Pept. Soc. 2016, 22, 280-289.

(177) Lovelace, E. S.; Armishaw, C. J.; Colgrave, M. L.; Wahlstrom, M. E.; Alewood, P. F.; Daly, N. L.; Craik, D. J. Cyclic MrIA: A Stable and Potent Cyclic Conotoxin with a Novel Topological Fold That Targets the Norepinephrine Transporter. J. Med. Chem. 2006, 49, 6561-6568.

(178) Hüttl, C.; Hettrich, C.; Riedel, M.; Henklein, P.; Rawel, H.; Bier, F. F. Development of Peptidyl Lysine Dendrons: 1,3-Dipolar Cycloaddition for Peptide Coupling and Antibody Recognition. Chem. Biol. Drug Des. 2015, 85, 565-573.

(179) Tang, W.; Becker, M. L. "Click" Reactions: A Versatile Toolbox for the Synthesis of Peptide-Conjugates. Chem. Soc. Rev. 2014, 43, 7013-7039.

(180) Nogueira-Librelotto, D. R.; Codevilla, C. F.; Farooqi, A.; Rolim, C. M. B. TransferrinConjugated Nanocarriers as Active-Targeted Drug Delivery Platforms for Cancer Therapy. Curr. Pharm. Des. 2017, 23, 454-466.

(181) Wang, W.; Kapur, A.; Ji, X.; Zeng, B.; Mishra, D.; Mattoussi, H. Multifunctional and High Affinity Polymer Ligand That Provides Bio-Orthogonal Coating of Quantum Dots. Bioconjug. Chem. 2016, 27, 2024-2036.

(182) Asadian-Birjand, M.; Biglione, C.; Bergueiro, J.; Cappelletti, A.; Rahane, C.; Chate, G.; Khandare, J.; Klemke, B.; Strumia, M. C.; Calderón, M. Transferrin Decorated Thermoresponsive Nanogels as Magnetic Trap Devices for Circulating Tumor Cells. Macromol. Rapid Commun. 2016, 37, 439-445.

(183) Jivan, F.; Yegappan, R.; Pearce, H.; Carrow, J. K.; McShane, M.; Gaharwar, A. K.; Alge, D. L. Sequential Thiol-Ene and Tetrazine Click Reactions for the Polymerization and Functionalization of Hydrogel Microparticles. Biomacromolecules 2016, 17, 3516-3523. 
(184) Martínez-Jothar, L.; Doulkeridou, S.; Schiffelers, R. M.; Sastre Torano, J.; Oliveira, S.; van Nostrum, C. F.; Hennink, W. E. Insights into Maleimide-Thiol Conjugation Chemistry: Conditions for Efficient Surface Functionalization of Nanoparticles for Receptor Targeting. J. Control. Release 2018, 282, 101-109.

(185) Estupiñán, D.; Bannwarth, M. B.; Mylon, S. E.; Landfester, K.; Muñoz-Espí, R.; Crespy, D. Multifunctional Clickable and Protein-Repellent Magnetic Silica Nanoparticles. Nanoscale 2016, 8, 3019-3030.

(186) Pathak, R. K.; McNitt, C. D.; Popik, V. V.; Dhar, S. Copper-Free Click-Chemistry Platform to Functionalize Cisplatin Prodrugs. Chem. Weinh. Bergstr. Ger. 2014, 20 , 6861-6865.

(187) Hui, J. Z.; Al Zaki, A.; Cheng, Z.; Popik, V.; Zhang, H.; Luning Prak, E. T.; Tsourkas, A. Facile Method for the Site-Specific, Covalent Attachment of Full-Length IgG onto Nanoparticles. Small Weinh. Bergstr. Ger. 2014, 10, 3354-3363.

(188) Ta, H. T.; Li, Z.; Hagemeyer, C. E.; Cowin, G.; Zhang, S.; Palasubramaniam, J.; Alt, K.; Wang, X.; Peter, K.; Whittaker, A. K. Molecular Imaging of Activated Platelets via Antibody-Targeted Ultra-Small Iron Oxide Nanoparticles Displaying Unique Dual MRI Contrast. Biomaterials 2017, 134, 31-42.

(189) Colombo, M.; Sommaruga, S.; Mazzucchelli, S.; Polito, L.; Verderio, P.; Galeffi, P.; Corsi, F.; Tortora, P.; Prosperi, D. Site-Specific Conjugation of ScFvs Antibodies to Nanoparticles by Bioorthogonal Strain-Promoted Alkyne-Nitrone Cycloaddition. Angew. Chem. Int. Ed Engl. 2012, 51, 496-499.

(190) Jeong, S.; Park, J. Y.; Cha, M. G.; Chang, H.; Kim, Y.-I.; Kim, H.-M.; Jun, B.-H.; Lee, D. S.; Lee, Y.-S.; et al. Highly Robust and Optimized Conjugation of Antibodies to Nanoparticles Using Quantitatively Validated Protocols. Nanoscale 2017, 9, 25482555.

(191) Lee-Montiel, F. T.; Li, P.; Imoukhuede, P. I. Quantum Dot Multiplexing for the Profiling of Cellular Receptors. Nanoscale 2015, 7, 18504-18514.

(192) Qiao, R.; Liu, C.; Liu, M.; Hu, H.; Liu, C.; Hou, Y.; Wu, K.; Lin, Y.; Liang, J.; Gao, M. Ultrasensitive in vivo Detection of Primary Gastric Tumor and Lymphatic Metastasis Using Upconversion Nanoparticles. ACS Nano 2015, 9, 2120-2129.

(193) Sanjaya, K. C.; Ranzoni, A.; Watterson, D.; Young, P.; Cooper, M. A. Evaluation of Direct versus Multi-Layer Passivation and Capture Chemistries for NanoparticleBased Biosensor Applications. Biosens. Bioelectron. 2015, 67, 769-774.

(194) Cserép, G. B.; Herner, A.; Kele, P. Bioorthogonal Fluorescent Labels: A Review on Combined Forces. Methods Appl. Fluoresc. 2015, 3, 042001.

(195) Koo, H.; Lee, S.; Na, J. H.; Kim, S. H.; Hahn, S. K.; Choi, K.; Kwon, I. C.; Jeong, S. Y.; Kim, K. Bioorthogonal Copper-Free Click Chemistry in vivo for Tumor-Targeted Delivery of Nanoparticles. Angew. Chem. Int. Ed Engl. 2012, 51, 11836-11840.

(196) Lamoot, A.; Uvyn, A.; Kasmi, S.; De Geest, B. G. Covalent Cell Surface Conjugation of Nanoparticles by a Combination of Metabolic Labeling and Click Chemistry. Angew. Chem. 2021, 133, 6390-6395.

(197) Chen, Y.; Cordero, J. M.; Wang, H.; Franke, D.; Achorn, O. B.; Freyria, F. S.; Coropceanu, I.; Wei, H.; Chen, O.; Mooney, D. J.; et al. A Ligand System for the Flexible Functionalization of Quantum Dots via Click Chemistry. Angew. Chem. Int. Ed Engl. 2018, 57, 4652-4656.

(198) Au, K. M.; Tripathy, A.; Lin, C. P.-I.; Wagner, K.; Hong, S.; Wang, A. Z.; Park, S. I. Bespoke Pretargeted Nanoradioimmunotherapy for the Treatment of Non-Hodgkin Lymphoma. ACS Nano 2018, 12, 1544-1563.

(199) Lu, G.; Zuo, L.; Zhang, J.; Zhu, H.; Zhuang, W.; Wei, W.; Xie, H.-Y. Two-Step TumorTargeting Therapy via Integrating Metabolic Lipid-Engineering with in Situ Click Chemistry. Biomater. Sci. 2020, 8, 2283-2288.

(200) Lee, S.; Yoon, H. I.; Na, J. H.; Jeon, S.; Lim, S.; Koo, H.; Han, S.-S.; Kang, S.-W.; Park, S.-J.; Moon, S.-H.; et al. In vivo Stem Cell Tracking with Imageable Nanoparticles That Bind Bioorthogonal Chemical Receptors on the Stem Cell Surface. Biomaterials 2017, 139, 12-29.

(201) Lim, S.; Kim, W.; Song, S.; Shim, M. K.; Yoon, H. Y.; Kim, B.-S.; Kwon, I. C.; Kim, K. Intracellular Uptake Mechanism of Bioorthogonally Conjugated Nanoparticles on 
Metabolically Engineered Mesenchymal Stem Cells. Bioconjug. Chem. 2021, 32, 199-214.

(202) Haun, J. B.; Devaraj, N. K.; Hilderbrand, S. A.; Lee, H.; Weissleder, R. Bioorthogonal Chemistry Amplifies Nanoparticle Binding and Enhances the Sensitivity of Cell Detection. Nat. Nanotechnol. 2010, 5, 660-665.

(203) Haun, J. B.; Devaraj, N. K.; Marinelli, B. S.; Lee, H.; Weissleder, R. Probing Intracellular Biomarkers and Mediators of Cell Activation Using Nanosensors and Bioorthogonal Chemistry. ACS Nano 2011, 5, 3204-3213.

(204) Chung, H. J.; Reiner, T.; Budin, G.; Min, C.; Liong, M.; Issadore, D.; Lee, H.; Weissleder, R. Ubiquitous Detection of Gram-Positive Bacteria with Bioorthogonal Magnetofluorescent Nanoparticles. ACS Nano 2011, 5, 8834-8841.

(205) Budin, G.; Chung, H. J.; Lee, H.; Weissleder, R. A Magnetic Gram Stain for Bacterial Detection. Angew. Chem. Int. Ed Engl. 2012, 51, 7752-7755.

(206) Tassa, C.; Liong, M.; Hilderbrand, S.; Sandler, J. E.; Reiner, T.; Keliher, E. J.; Weissleder, R.; Shaw, S. Y. On-Chip Bioorthogonal Chemistry Enables Immobilization of in Situ Modified Nanoparticles and Small Molecules for Label-Free Monitoring of Protein Binding and Reaction Kinetics. Lab. Chip 2012, 12, 3103-3110.

(207) Ghazani, A. A.; Pectasides, M.; Sharma, A.; Castro, C. M.; Mino-Kenudson, M.; Lee, H.; Shepard, J.-A. O.; Weissleder, R. Molecular Characterization of Scant Lung Tumor Cells Using Iron-Oxide Nanoparticles and Micro-Nuclear Magnetic Resonance. Nanomedicine Nanotechnol. Biol. Med. 2014, 10, 661-668.

(208) Lu, G.; Li, F.; Zhang, F.; Huang, L.-L.; Zhang, L.; Lv, Y.; Wei, W.; Xie, H.-Y. Amplifying Nanoparticle Targeting Performance to Tumor via Diels-Alder Cycloaddition. Adv. Funct. Mater. 2018, 1707596.

(209) Yoo, J.; Choi, S.; Son, J.; Yi, G.; Kim, E.; Koo, H. Click Chemistry-Mediated TumorTargeting of SN38-Loaded Nanoparticles Using Trastuzumab. Biochem. Biophys. Res. Commun. 2019, 515, 207-213.

(210) Agasti, S. S.; Liong, M.; Tassa, C.; Chung, H. J.; Shaw, S. Y.; Lee, H.; Weissleder, R. Supramolecular Host-Guest Interaction for Labeling and Detection of Cellular Biomarkers. Angew. Chem. Int. Ed Engl. 2012, 51, 450-454.

(211) O'Brien, P. J.; Elahipanah, S.; Rogozhnikov, D.; Yousaf, M. N. Bio-Orthogonal Mediated Nucleic Acid Transfection of Cells via Cell Surface Engineering. ACS Cent. Sci. 2017, 3, 489-500.

(212) Du, L.; Qin, H.; Ma, T.; Zhang, T.; Xing, D. In vivo Imaging-Guided Photothermal/Photoacoustic Synergistic Therapy with Bioorthogonal Metabolic Glycoengineering-Activated Tumor Targeting Nanoparticles. ACS Nano 2017, 11, 8930-8943.

(213) Mao, D.; Hu, F.; Kenry, J.; S.; Wu, W.; Ding, D.; Kong, D.; Liu, B. Metal-OrganicFramework-Assisted In vivo Bacterial Metabolic Labeling and Precise Antibacterial Therapy. Adv. Mater. 2018, 1706831.

(214) Lee, S. B.; Kim, H. L.; Jeong, H.-J.; Lim, S. T.; Sohn, M.-H.; Kim, D. W. Mesoporous Silica Nanoparticle Pretargeting for PET Imaging Based on a Rapid Bioorthogonal Reaction in a Living Body. Angew. Chem. Int. Ed. 2013, 52, 10549-10552.

(215) Jeong, H. J.; Yoo, R. J.; Kim, J. K.; Kim, M. H.; Park, S. H.; Kim, H.; Lim, J. W.; Do, S. H.; Lee, K. C.; Lee, Y. J.; et al. Macrophage Cell Tracking PET Imaging Using Mesoporous Silica Nanoparticles via in vivo Bioorthogonal F-18 Labeling. Biomaterials 2019, 199, 32-39.

(216) Tu, Y.; Dong, Y.; Wang, K.; Shen, S.; Yuan, Y.; Wang, J. Intercellular Delivery of Bioorthogonal Chemical Receptors for Enhanced Tumor Targeting and Penetration. Biomaterials 2020, 259, 120298.

(217) Lee, S.; Koo, H.; Na, J. H.; Han, S. J.; Min, H. S.; Lee, S. J.; Kim, S. H.; Yun, S. H.; Jeong, S. Y.; Kwon, I. C.; et al. Chemical Tumor-Targeting of Nanoparticles Based on Metabolic Glycoengineering and Click Chemistry. ACS Nano 2014, 8, 2048-2063.

(218) Yoon, H. Y.; Shin, M. L.; Shim, M. K.; Lee, S.; Na, J. H.; Koo, H.; Lee, H.; Kim, J.-H.; Lee, K. Y.; Kim, K.; et al. Artificial Chemical Reporter Targeting Strategy Using Bioorthogonal Click Reaction for Improving Active-Targeting Efficiency of Tumor. Mol. Pharm. 2017, 14, 1558-1570. 
(219) Brand, C.; lacono, P.; Pérez-Medina, C.; Mulder, W. J. M.; Kircher, M. F.; Reiner, T. Specific Binding of Liposomal Nanoparticles through Inverse Electron-Demand DielsAlder Click Chemistry. ChemistryOpen 2017, 6, 615-619.

(220) Denk, C.; Svatunek, D.; Mairinger, S.; Stanek, J.; Filip, T.; Matscheko, D.; Kuntner, C.; Wanek, T.; Mikula, H. Design, Synthesis, and Evaluation of a Low-MolecularWeight ${ }^{11}$ C-Labeled Tetrazine for Pretargeted PET Imaging Applying Bioorthogonal in vivo Click Chemistry. Bioconjug. Chem. 2016, 27, 1707-1712.

(221) van Onzen, A. H. A. M.; Rossin, R.; Schenning, A. P. H. J.; Nicolay, K.; Milroy, L.-G.; Robillard, M. S.; Brunsveld, L. Tetrazine-Trans -Cyclooctene Chemistry Applied to Fabricate Self-Assembled Fluorescent and Radioactive Nanoparticles for in vivo Dual Mode Imaging. Bioconjug. Chem. 2019, 30, 547-551.

(222) Keinänen, O.; Mäkilä, E. M.; Lindgren, R.; Virtanen, H.; Liljenbäck, H.; Oikonen, V.; Sarparanta, M.; Molthoff, C.; Windhorst, A. D.; Roivainen, A.; et al. Pretargeted PET Imaging of Trans -Cyclooctene-Modified Porous Silicon Nanoparticles. ACS Omega 2017, 2, 62-69.

(223) Qiao, J.; Tian, F.; Deng, Y.; Shang, Y.; Chen, S.; Chang, E.; Yao, J. Bio-Orthogonal Click-Targeting Nanocomposites for Chemo-Photothermal Synergistic Therapy in Breast Cancer. Theranostics 2020, 10, 5305-5321.

(224) Wang, H.; Tang, L.; Liu, Y.; Dobrucka, I. T.; Dobrucki, L. W.; Yin, L.; Cheng, J. In vivo Targeting of Metabolically Labeled Cancers with Ultra-Small Silica Nanoconjugates. Theranostics 2016, 6, 1467-1476.

(225) Wei, R.; Dong, Y.; Tu, Y.; Luo, S.; Pang, X.; Zhang, W.; Yao, W.; Tang, W.; Yang, H.; Wei, X.; et al. Bioorthogonal Pretargeting Strategy for Anchoring Activatable Photosensitizers on Plasma Membranes for Effective Photodynamic Therapy. ACS Appl. Mater. Interfaces 2021, 13, 14004-14014.

(226) Zhang, W.; Deng, W.; Zhang, H.; Sun, X.; Huang, T.; Wang, W.; Sun, P.; Fan, Q.; Huang, W. Bioorthogonal-Targeted 1064 Nm Excitation Theranostic Nanoplatform for Precise NIR-Ila Fluorescence Imaging Guided Efficient NIR-II Photothermal Therapy. Biomaterials 2020, 243, 119934.

(227) Perez-Medina, C.; Abdel-Atti, D.; Zhang, Y.; Longo, V. A.; Irwin, C. P.; Binderup, T.; Ruiz-Cabello, J.; Fayad, Z. A.; Lewis, J. S.; Mulder, W. J. M.; et al. A Modular Labeling Strategy for In vivo PET and Near-Infrared Fluorescence Imaging of Nanoparticle Tumor Targeting. J. Nucl. Med. 2014, 55, 1706-1711.

(228) Du, L.; Qin, H.; Ma, T.; Zhang, T.; Xing, D. In vivo Imaging-Guided Photothermal/Photoacoustic Synergistic Therapy with Bioorthogonal Metabolic Glycoengineering-Activated Tumor Targeting Nanoparticles. ACS Nano 2017, 11, 8930-8943.

(229) Lee, S.; Jung, S.; Koo, H.; Na, J. H.; Yoon, H. Y.; Shim, M. K.; Park, J.; Kim, J.-H.; Lee, S.; Pomper, M. G.; et al. Nano-Sized Metabolic Precursors for Heterogeneous Tumor-Targeting Strategy Using Bioorthogonal Click Chemistry in vivo. Biomaterials 2017, 148, 1-15.

(230) Xie, X.; Li, B.; Wang, J.; Zhan, C.; Huang, Y.; Zeng, F.; Wu, S. Bioorthogonal Nanosystem for Near-Infrared Fluorescence Imaging and Prodrug Activation in Mouse Model. ACS Mater. Lett. 2019, 1, 549-557.

(231) Xie, X.; Li, B.; Wang, J.; Zhan, C.; Huang, Y.; Zeng, F.; Wu, S. Tetrazine-Mediated Bioorthogonal System for Prodrug Activation, Photothermal Therapy, and Optoacoustic Imaging. ACS Appl. Mater. Interfaces 2019, 11, 41875-41888.

(232) Liu, Y.; Hou, W.; Sun, H.; Cui, C.; Zhang, L.; Jiang, Y.; Wu, Y.; Wang, Y.; Li, J.; Sumerlin, B. S.; et al. Thiol-Ene Click Chemistry: A Biocompatible Way for Orthogonal Bioconjugation of Colloidal Nanoparticles. Chem. Sci. 2017, 8, 61826187.

(233) Ke, P. C.; Lin, S.; Parak, W. J.; Davis, T. P.; Caruso, F. A Decade of the Protein Corona. ACS Nano 2017, 11, 11773-11776.

(234) Mirshafiee, V.; Mahmoudi, M.; Lou, K.; Cheng, J.; Kraft, M. L. Protein Corona Significantly Reduces Active Targeting Yield. Chem. Commun. 2013, 49, 2557-2559.

(235) Jiang, C.; Wang, G.; Hein, R.; Liu, N.; Luo, X.; Davis, J. J. Antifouling Strategies for Selective In vitro and In vivo Sensing. Chem. Rev. 2020, 120, 3852-3889. 
(236) Moyano, D. F.; Saha, K.; Prakash, G.; Yan, B.; Kong, H.; Yazdani, M.; Rotello, V. M. Fabrication of Corona-Free Nanoparticles with Tunable Hydrophobicity. ACS Nano 2014, 8, 6748-6755.

(237) Affonso de Oliveira, J. F.; Scheffer, F. R.; Landis, R. F.; Teixeira Neto, É.; Rotello, V. M.; Cardoso, M. B. Dual Functionalization of Nanoparticles for Generating CoronaFree and Noncytotoxic Silica Nanoparticles. ACS Appl. Mater. Interfaces 2018, 10 , 41917-41923.

(238) Zhang, X.; Liu, Y.; Gopalakrishnan, S.; Castellanos-Garcia, L.; Li, G.; Malassiné, M.; Uddin, I.; Huang, R.; Luther, D. C.; Vachet, R. W.; et al. Intracellular Activation of Bioorthogonal Nanozymes through Endosomal Proteolysis of the Protein Corona. ACS Nano 2020, 14, 4767-4773. 
TOC Graphic

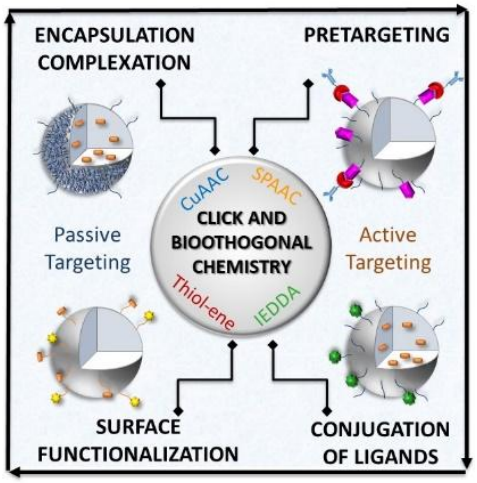

